\title{
NEAR INFRARED FLUORESCENT SENSORS AND THEIR USE IN VIVO
}

\author{
A Thesis presented to \\ the Faculty of the Graduate School \\ at the University of Missouri
}

In Partial Fulfillment

of the Requirements for the Degree

Doctor of Philosophy

by
NICHOLAS P. COOLEY

Dr. Timothy E. Glass, Dissertation Supervisor

MAY 2017 
The undersigned, appointed by the Dean of the Graduate School, have examined the dissertation entitled:

\section{NEAR INFRARED FLUORESCENT SENSORS AND THEIR USE IN VIVO}

presented by Nicholas Cooley, a candidate for the degree of Doctor of Philosophy and hereby certify that, in their opinion, it is worthy of acceptance.

Dr. Timothy Glass

Dr. Susan Lever

Dr. Kent Gates

Dr. David Kline 


\section{ACKNOWLEDGMENTS}

This dissertation was made possible through the mentorship and support of many

individuals. My parents, Frank and Theresa have been tremendously supportive throughout my entire time in graduate school. My brothers, Michael, Patrick and Jason, and my sister in law Renee, I owe you deeply for your advice, understanding, and patience in putting up with the occasional frustration induced monologue.

My friends and mentors from Ohio State, without whose input and support I never would have made it to graduate school; Pat Kington, Dan Culicover, Chris Swartz, Marco Benedetti, Ross Chasko, Tino Benedetti, Dr. Nicholas Libertine, Dr. Ryan Griffith, Dr. Chris Callam, Dr. Greg Booton, and Sara and Mark Neihus. To my friends in Missouri, and elsewhere I thank you everything; Matt Schmidt, Sam Cook, Travis Pemberton, Anahita Zare, Adrian Laciak, and Dr. Chris Patane.

Thank you Hannah, and Winston for helping to curb my more extreme swings in both optimism and pessimism.

Finally I would like to thank my advisor, Dr. Timothy Glass, for his guidance, mentorship, and most importantly patience, throughout my graduate education. I would also like to thank my committee, Dr. Kent Gates, Dr. Susan Lever, and Dr. David Kline, for their time and attention. I would like to thank our collaborators Dr. Ken Meissner and Dr. Mark Milanick for their patience and input, without which much of this work would not have been completed. I owe tremendous thanks to the graduate students who helped mentor me in my early years of graduate school; Chun Ren, Shaohui Zhang, Chad Cooley, Ken Hettie, Jessica Klockow, and Xiaole Shao. And the rest of the Glass Group, C.W. Littlefield, Hamid Sepasizangabadi, Ming Xu, 
Le Zhang, Ashley Knulty, and Anusha Bade, I wish you the best. I hope that my advice has been helpful, and that your projects are successful. 


\section{TABLE OF CONTENTS}

ACKNOWLEDGMENTS ................. ii

LIST OF TABLES ..................... . vi

LIST OF FIGURES ................... . vii

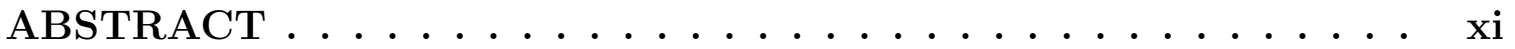

\section{CHAPTER}

1 Introduction ...................... 1

1.1 Fluorescence . . . . . . . . . . . . . . . . . . 1

1.1.1 Fluorescence as a Tool for Visualization . . . . . . . . . . . . 4

1.1.2 Sensing of Supramolecular Interactions . . . . . . . . . . . . 7

1.1 .3 Binding ............................ 11

1.1.4 Biologically Relevant Analytes . . . . . . . . . . . . . 15

1.1.5 Sugars ....................... 16

1.1.6 Aggregation ................... 16

2 pH Sensing with Tetra Sulfonated Cyanine Compounds . . . . . 19

$2.1 \mathrm{pH}$ in vivo . . . . . . . . . . . . . . . . . . 19

2.1.1 pH Sensing in The Literature . . . . . . . . . . . . . . 20

2.1.2 Tetra-sulfonated Amino-Cyanine $\mathrm{pH}$ Sensors . . . . . . . . . . 22

2.1.3 Unsuccessful Modifications . . . . . . . . . . . . . . . . . . 31

2.2 Future Work . . . . . . . . . . . . . . . . . . 34 
2.2.1 pH Sensing as a Route Sensing Sugars . . . . . . . . . . . . 37

3 Near IR Sugar Sensing ................... 40

3.1 Fluorescent Chemical Detection of Sugars . . . . . . . . . . . . . 40

3.2 NIR Sugar Sensing . . . . . . . . . . . . . . . . . . 47

3.2.1 Designing a NIR Cyanine Based Sugar Sensor . . . . . . . . . 48

3.2.2 Attempts at Building Complex Sugar Binding Motifs . . . . . 51

3.3 Future Work . . . . . . . . . . . . . . . 56

4 Erythrocyte Loading .................... 58

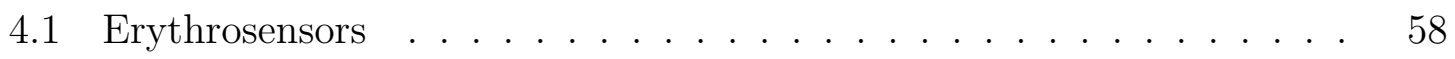

4.1.1 Erythrocyte Loading Methods . . . . . . . . . . . . . 59

4.1.2 Applications ..................... 61

4.2 Erythrocyte Loading With Tetra-Sulfonated Cyanine Derivates . . . . 63

4.2.1 Animal Studies . . . . . . . . . . . . . . . 73

4.2.2 Future Work. . . . . . . . . . . . . . . 78

\section{APPENDIX}

A Experimental Procedures and Characterization Data . . . . . 81

A.1 Experimental Procedures . . . . . . . . . . . . . 81

A.2 Compound Characterization ................ 85

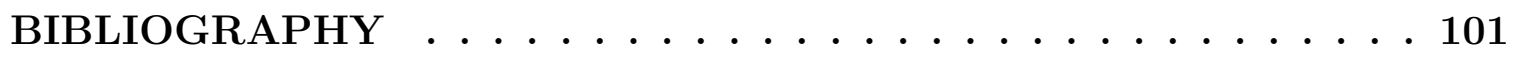

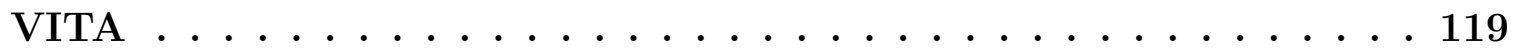




\section{LIST OF TABLES}

Table $\quad$ Page

1.1 Table of Interaction Forces . . . . . . . . . . . . . . . . . . . 13

$2.1 \mathrm{pK}_{\mathrm{a}}$ of 11 by Concentration . . . . . . . . . . . 26

$2.2 \mathrm{pK}_{\mathrm{a}}$ of 12 by Concentration $\ldots \ldots \ldots \ldots \ldots \ldots$

$2.3 \mathrm{pK}_{\mathrm{a}}$ of 14 by Concentration . . . . . . . . . . . . 30

3.1 Sugar Sensors . . . . . . . . . . . . . . . . . . . 46

3.2 Binding and Saturation Constants for $45 \ldots \ldots \ldots \ldots$

4.1 Time Constants From in vivo Experiments . . . . . . . . . . 78 


\section{LIST OF FIGURES}

$\begin{array}{ll}\text { Figure } & \text { Page }\end{array}$

1.1 Jablonksi Diagram of Fluorescence and Phosphorescence . . . . . . . 2

1.2 Selected Fluorophores and the Visible Spectrum . . . . . . . . . . . 3

1.3 Fluorescence Emission . . . . . . . . . . . . . . . . 4

1.4 Fluorescent Probes in Cells . . . . . . . . . . . . . . . 5

1.5 A Fluorescently Labeled Tumor . . . . . . . . . . . . . . . . . . 6

1.6 Light Scattering in Tissue . . . . . . . . . . . . . . . . 7

1.7 Coumarin Aldehyde - Amine Binding . . . . . . . . . . . . 8

1.8 Si-Rhodamine Based Calcium Sensor . . . . . . . . . . . . . . 9

1.9 PET Quenching Mechanism ................ . 10

1.10 FRET . . . . . . . . . . . . . . . . . 11

1.11 FRET Based Probe for $\mathrm{H}_{2} \mathrm{O}_{2} \quad \ldots \ldots \ldots$

1.12 One Site Binding Isotherm . . . . . . . . . . . . . . 14

1.13 Sigmoidal Binding Isotherm . . . . . . . . . . . . . . 15

1.14 Aggregation Models . . . . . . . . . . . . . . . 17

1.15 Emission and Absoprtion in Aggregates . . . . . . . . . . . . . 18

$2.1 \mathrm{BCECF} \ldots \ldots \ldots \ldots \ldots$ 


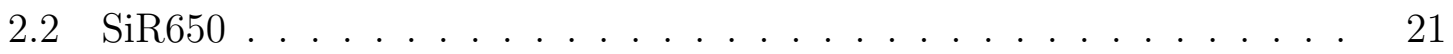

$2.3 \mathrm{pH}$ Sensitive H-Cyanines . . . . . . . . . . . . . . . . . . . 21

$2.4 \mathrm{pH}$ Sensitive Cyanines . . . . . . . . . . . . . . . . . . 22

2.5 Synthesis of Methyl-Piperazine appended Cyanine . . . . . . . . 23

$2.6 \mathrm{pH}$ Titration of $\mathbf{1 1}$ by Absorption Spectroscopy . . . . . . . . . . 24

$2.7 \mathrm{pH}$ Titration of $\mathbf{1 1}$ by Fluorescence Spectroscopy $\ldots \ldots \ldots \ldots$

2.8 Synthesis of Piperidine appended Cyanine $\ldots \ldots \ldots$

$2.9 \mathrm{pH}$ Titration of $\mathbf{1 2}$ by Fluorescence Spectroscopy $\ldots \ldots \ldots \ldots$

2.10 Synthesis of Piperidine appended Cyanine $\ldots \ldots \ldots \ldots$

2.11 Synthesis of Phenyl-methylpiperazine appended Cyanine $\ldots . . .29$

2.12 Fluorescence and Absorbance $\mathrm{pH}$ Titration of $\mathbf{1 4} \ldots \ldots$

2.13 Proposed Methypiperazine-Cyanines . . . . . . . . . . . 33

2.14 Proposed Test Modifications of $10 \ldots \ldots \ldots$

2.15 Sonogashira Chemistry on $10 \ldots \ldots \ldots \ldots$

2.16 Attempts at Alkylboronic Ester Coupling to $10 \ldots \ldots$

2.17 Potential Suzuki Coupling Partners . . . . . . . . . . . . 36

2.18 Potential Buchwald-Hartwig Coupling Partners . . . . . . . . 36

2.19 Potential Buchwald-Hartwig Coupling Partners . . . . . . . . . 37

$2.20 \mathrm{pH}$ PET Quenching . . . . . . . . . . . . . . . . . . 37

2.21 Phenylboronic Acid PET Quenching . . . . . . . . . . 38

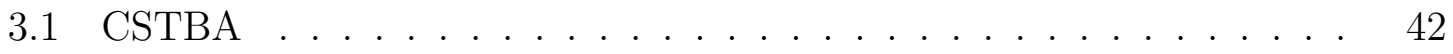

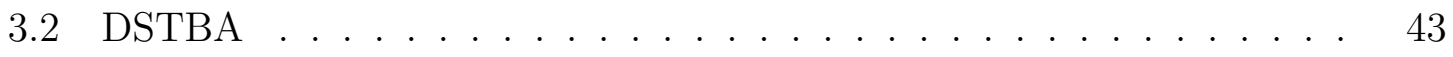

3.3 Shinkai's Anthracene Based PET System . . . . . . . . . . . . 43

3.4 Shinkai's Anthracene Based Bisboronic Acid Receptor . . . . . . . . . 44 
3.5 Intramolecular Energy Transfer Based Glucose Sensors . . . . . . . . 44

3.6 Drueckhammer's Glucose Sensor . . . . . . . . . . . . . . 45

3.7 NIR Glucose Sensitive Bioconjugate . . . . . . . . . . . . . . . . 47

3.8 Cyanine Based Sugar Sensor . . . . . . . . . . . . . . . . . . 48

3.9 Absorbance Titration of $\mathbf{4 5}$ with Fructose . . . . . . . . . . . . . 49

3.10 Fluorescence Titrations of $\mathbf{4 5}$ with Glucose and Fructose . . . . . . . 50

3.11 Cyanine Based Sugar Sensor 46 . . . . . . . . . . . . . . . . 52

3.12 Bis-secondary amine intermediate a . . . . . . . . . . . 52

3.13 Unsuccessful Bisboronic Acid 48 . . . . . . . . . . . . . . . 53

3.14 Unsuccessful Bis-secondary Amine Intermediate 49 . . . . . . . . . 54

3.15 Unsuccessful Phenylboronic Acid Compound 50 . . . . . . . . . . . 54

3.16 Unsuccessful Phenylboronic Acid Compound 52 . . . . . . . . . . . 55

3.17 Potential Intermediates for achieving a Bis-boronic acid based receptor 57

4.1 Generically Erythrosensor . . . . . . . . . . . . . . . 59

4.2 Generic Ghosting Scheme . . . . . . . . . . . . . . . . 60

4.3 Dexamethasone Phosphate Hydrolysis . . . . . . . . . . . . . . . 62

4.4 Sortase Mediated Erythrocyte Modification . . . . . . . . . . . 63

4.5 Intstrument for taking Fluorescence Measurements in vivo . . . . . . 64

4.6 Schematic of Instrument for taking Fluorescence Measurements in vivo 65

4.7 Erythrocyte Loading . . . . . . . . . . . . . . . . . . . . . 66

4.8 Fluorometer measurements of diluted Erythrocytes, and Low Hemoglobin Sensor Loaded Erythrocytes . . . . . . . . . . . . . . . 67

$4.9 \mathrm{pH}$ Titration of Dilution Loaded Erythrocytes Containing Sensor 11 . 68 
4.10 Fluorometer Measurements of Supernatant buffer from Erthrocyte Washes in low hemoglobin hypo-osmotic loading . . . . . . . . . . . . . . 69

4.11 Fluorometer measurements of diluted Erythrocytes, and Normal Hemoglobin Sensor Loaded Erythrocytes . . . . . . . . . . . . . . . . . 70

4.12 Fluorometer Measurements of Wash Step Supernatants and Erythrocyte Lysis for Dialysis Loading . . . . . . . . . . . . . . . . . . . . . . 71

4.13 Fluorometer Measurements of Supernatant buffer from Erthrocyte Washes in Dialysis Loading . . . . . . . . . . . . . . . . . . . . . . 72

4.14 Fluorescence measurements by Meissner and Ritter's system . . . . . 73

4.15 Exponential Decay Curve . . . . . . . . . . . . . . . 75

4.16 Fluorescence Time Course of Sensor Loaded Erythrocytes in vivo . . 76

4.17 Fluorescence Time Course of Sensor Loaded Erythrocytes in vivo . . 77

4.18 SEM Images of Erythrocytes . . . . . . . . . . . . . . . . 79 


\begin{abstract}
In an effort to design near-infrared (NIR), water soluble glucose sensors, several pH sensitive NIR cyanine derivates were designed and synthesized to provide insight into the viability of the cyanine platform as the fluorophore core for performing minimally invasive long term glucose monitoring in vivo. Many previous efforts to build effective fluorescent sensors for glucose have provided guidance towards the architecture of binding groups and fluorescent response required to achieve this goal, but have not provided appropriate solubility, or excitation and emission characteristics for in vivo sensing. In an effort to address the aqueous solubility of the highly rigid cyanine platform, a tetra sulfonated core was chosen for this work. Though fully water soluble, pH sensitive derivates still showed some aggregation characteristics. Simple sugarbinding boronic acid derivatives showed appropriate fluorescent responses, but poor binding. Efforts to improve binding through synthesis of bis-boronic acid compounds proved elusive.
\end{abstract}




\section{Chapter 1}

\section{Introduction}

\section{$1.1 \quad$ Fluorescence}

Organic compounds with conjugated $\pi$ systems can absorb photons, which puts them into an excited state. As this state is not stable, the compound must then de-excite. There are many paths of de-excitation; one such is the emission of a photon of energy equivalent to the transition between the excited state of the compound and the ground state. Compounds that undergo this process are called fluorophores. First observed in 1845 by Sir John Frederick William Herschel with quinine dissolved in tonic water, the study of fluorescence, and the design and study of compounds that use fluorescence as a tool is currently a field of great depth and breadth [1, 2].

The central qualities of a fluorophore are its absorption, and its fluorescent emission. The photon absorption of a compound is governed by the structure and arrangement of the compounds $\pi$ system. Photon absorption can by broken down into an 
absorption spectrum that maps photon absorption across wavelength, and molar absorptivity. Compounds with large $\pi$ systems absorb photons of different wavelengths with varying degrees of efficiency; the degrees of these differences can be highly dependent upon pi system in question. Molar absorptivity is an intrinsic property of any chemical species that absorbs light, regardless of whether that species returns to its electronic ground state through fluorescence or another process, it is the measure of how well a compound attenuates light at a specific wavelength, see Figure 1.1 [1].

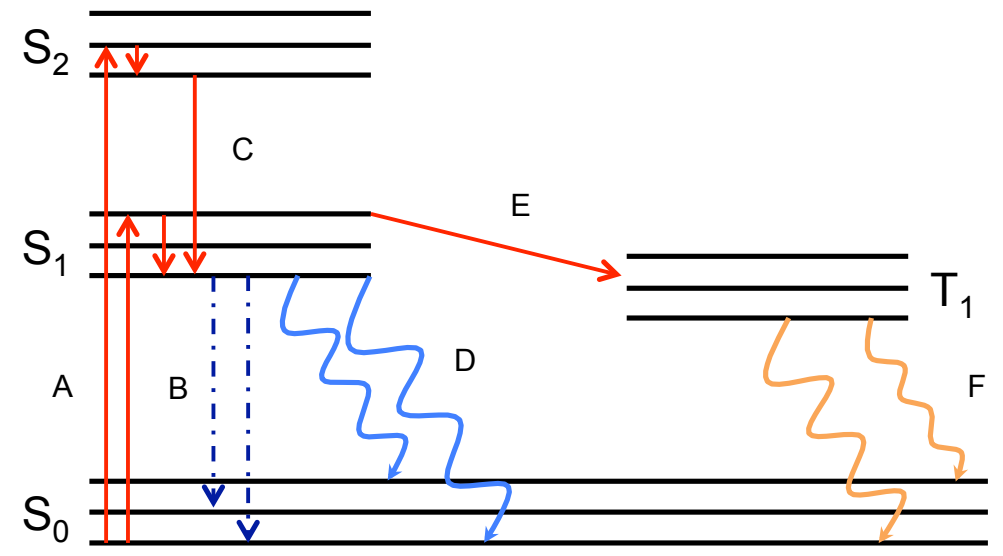

Figure 1.1: Jablonksi Diagram: A - Absorption of a Photon, B - Non-Radiative Decay, C - Internal Conversion, D - Fluorescence, E - Intersystem Crossing, F Phosphorescence, $\mathrm{S}_{0}$ - Electronic Ground State, $\mathrm{S}_{1}$ and $\mathrm{S}_{2}$ - Singlet Excited States, $\mathrm{T}_{1}$ - Triplet Excited State

Fluorescent emission, similarly governed by structure can be again broken down into a spectrum, mapping the wavelengths of emission, and into quantum yield. Directly tied to the molar absorptivity, the quantum yield of a fluorophore is a direct relation of how many photons end up being emitted for every photon absorbed. Some fluorophores present exceptionally high quantum yields, while others present very low ones.

Fluorescent emission is one of the possible ways that compounds can de-excite, 


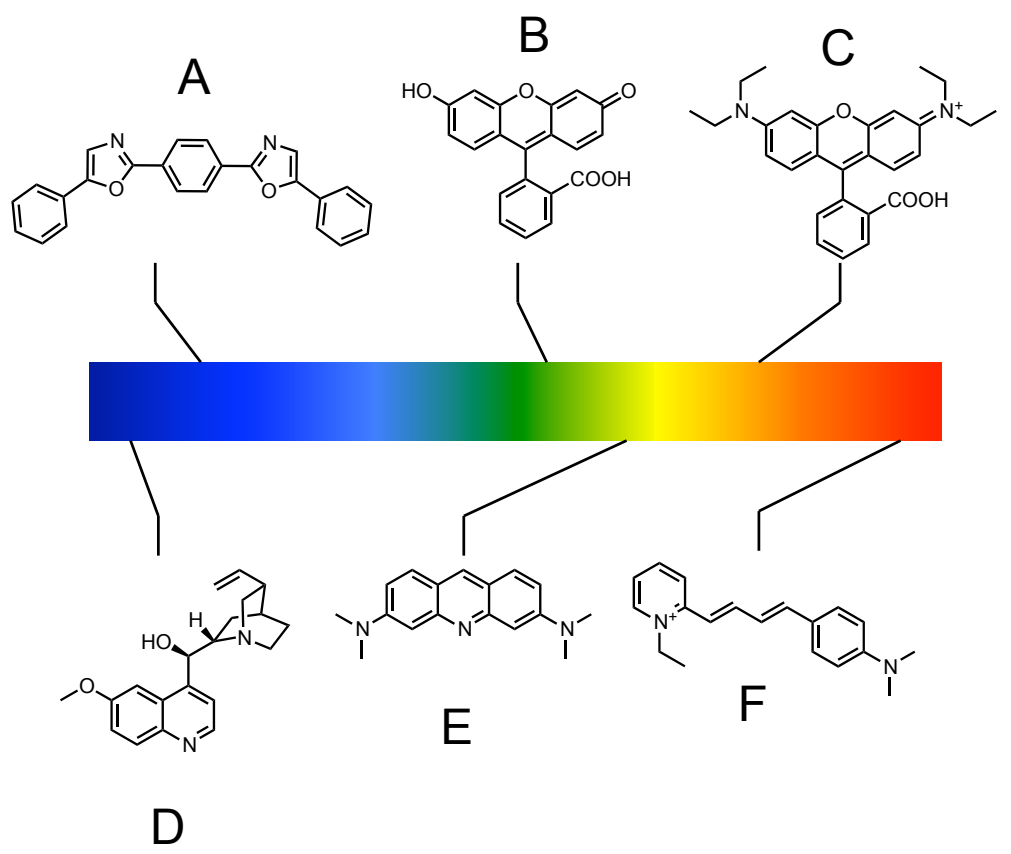

Figure 1.2: Visible Spectrum and Selected Fluorophores: A - POPOP, B - Quinine, C - Fluorescein, D - Acridine Orange, E - Rhodamine B, F - Pyridine 1

compounds with large quantum yields de-excite largely through the emission of a photon, while compounds with poor quantum yields de-excite largely through nonradiative decay pathways. Another possibility is that the energy of the absorbed photon can undergo intersystem crossing to fill a triplet state, which it then photodeexcites from at on a significantly slower time scale. This phenomenon is phosphorescence, and is responsible for such things as glow-in-the-dark materials.

There are many commercially available fluorophores, and even more which are accessible to chemists with the appropriate means and capability in the lab. Two of the defining characteristics of fluorophores are their absorbance, and emission properties. How well they absorb and emit photons is important, but also where they effectively absorb and emit photons is of importance. Chemical structures of fluorophores play 
significant roles in this, and because of this, there is tremendous variety in fluorophore type, and structure, Figure 1.2.

\subsubsection{Fluorescence as a Tool for Visualization}

Because fluorescence emission is environmentally and structurally sensitive it allows for the use of fluorescence as a tool for monitoring intramolecular phenomenon. Fluorescent sensors and probes can monitor a variety of changes in systems, including but not limited to: changes in oxidation states, increases or decreases in concentration of analytes in solution, changes in local $\mathrm{pH}$, and monitoring protein-protein interactions $[3,4,5]$. This works through the fluorescence output being monitored as a signal output. This signal output can be quantified and allows for visualization and quantification of phenomena in cuvette, Figure 1.3.

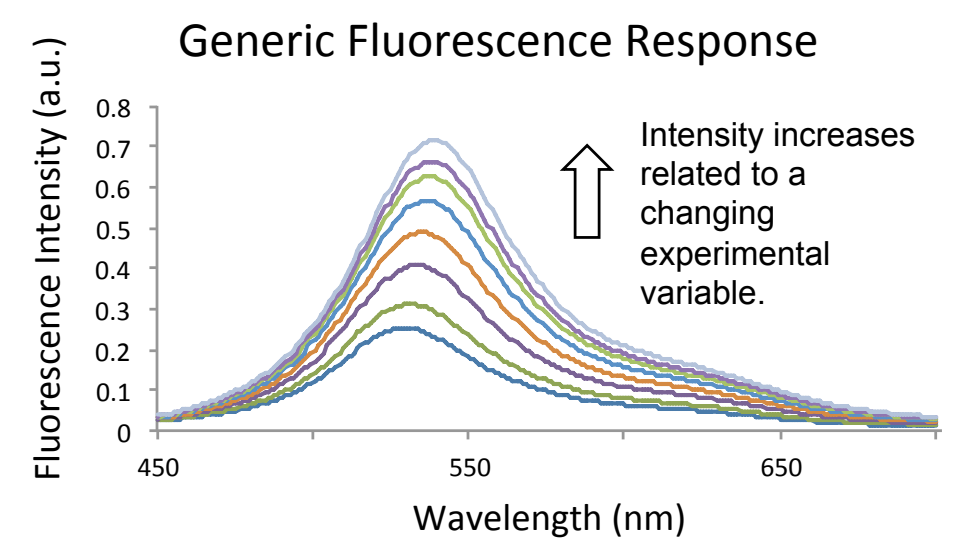

Figure 1.3: Stacked fluorescence emission spectra allow for visualization and quantification of emission intensities.

Cuvette experiments provide valuable information, but the goal of most fluorescent sensors are in vivo detection experiments. If the fluorescent sensor whose response 
is described in Figure 1.3 were designed to respond to Zinc, which is an essential neurotransmitter in healthy brain tissue, it would need to be tested in a cell model. That test would provide information somewhat like that shown in, Figure 1.4

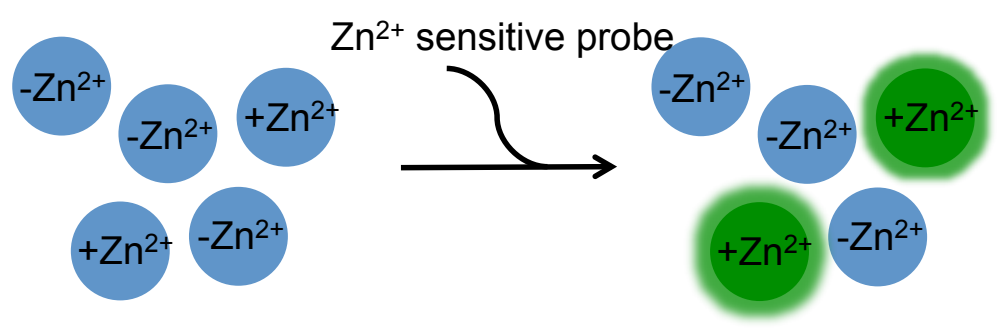

Figure 1.4: A fluorescent sensor designed to respond to zinc would ideally produce emission signals that allow for differentiation between $\mathrm{Zn}^{2+}$ containing cells, and those without.

Work by Komatsu et al. describes a fluorescent sensor used to quantify zinc, first in solution in cuvette, and then in model cells, and then in vivo in rat hippocampus cells [6]. Figures 1.3, 1.4 provide simple illustrations of how this data can be presented. Figure 1.3 illustrates the results of cuvette experiments are performed on a fluorometer, while Figure 1.4 provides a cartoon illustration of a fluorescence microscopy experiment.

Achilefu's group achieves specific visualization of different types of tumors using a cyanine based fluorophore which is $\mathrm{pH}$ sensitive, and injected into the mouse model, Figure 1.5. Over time the compound accumulates in various tissues, however because it is $\mathrm{pH}$ sensitive it gives distinct fluorescence responses to accumulation in tumors vs accumulation in healthy tissue. Further work by Achilefu's group shows that effective processing and quantification of the images captured from these in vivo experiments is itself not a trivial task. All types of tissue absorb light and that absorption can affect signal output from compounds used in vivo. Subjecting a well characterized 
NIR probe, accumulated in tumors, to a series of control experiments and analysis allowed for more accurate determination of signal origin location [7]. This is necessary as tissue is a scattering environment. NIR Fluorophores with functional groups that accurately localize the compound to tumors, or to specific organs can be used for visualization. This could serve solely to locate tumors in control experiments, for tumor cell quantification as a precursor to surgery, or as a visualization aid in determining the efficacy of drug delivery system, or targeting "warhead" groups designed to localization attached groups to tumors.

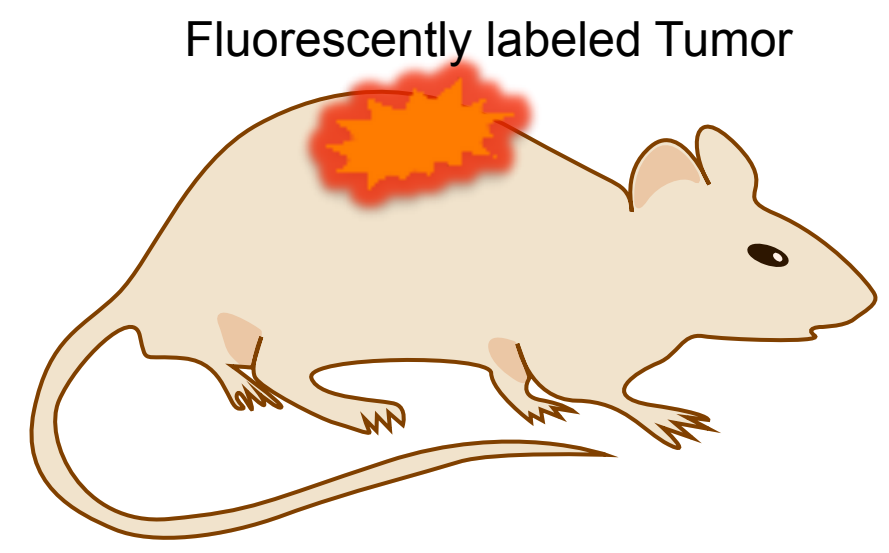

Figure 1.5: A successful fluorescent label for a tumor will show high intensity fluorescence when localized in the tumor, and little background fluorescence from the rest of the animal model.

Fluorescent probes are often also used for tumor visualization to either aid in diagnosis of tumors, or surgical removal. The difference in parameters for experiments in vivo from in vitro are significant. Work in cell cultures, though not trivial is simpler than designing a fluorescent sensors for in vivo work, one constant concern is that tissue is a scattering medium for light. This both limits the emission and excitation wavelengths that can be effective in vivo, and complicates signal detection. Figure 
1.6 graphically depicts the signal convolution caused by tissue scattering on signal output.

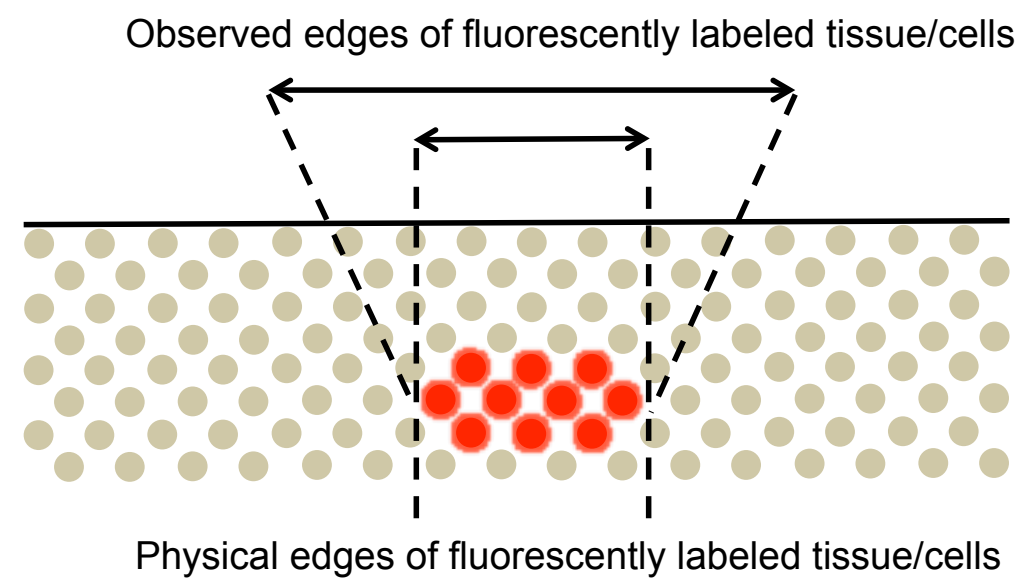

Figure 1.6: Depiction of light scattering in tissue and its effect on signal output.

\subsubsection{Sensing of Supramolecular Interactions}

There are a variety of ways through which fluorescence can be utilized as a sensing mechanism. Fluorophores are generally constructed around an internal push-pull system, where a conjugated system contains either formally charged groups, or polarized functional groups which form a 'Donor $-\pi$ - Acceptor' construct. Chemical events that effect the electronics of this construct cause a change in the fluorescent emission of the fluorophore. These events manifests as either a change in quantum yield, or a change in emission wavelength. These changes in emission intensity or shifts in emission max can then be quantified to provide information about the event that caused a change in the electronics of the conjugated system. In work by the Glass group this can be seen when a coumarin aldehyde, with an attached electron donor group - usually an amine - reacts with a primary amine to form a stable iminium 
compound see Figure 1.7 [8]. The iminium compound presents a different absorbance profile from the aldehyde, and the extent of the binding reaction can be measured by exciting the new absorbance max, and following the change in fluorescence.

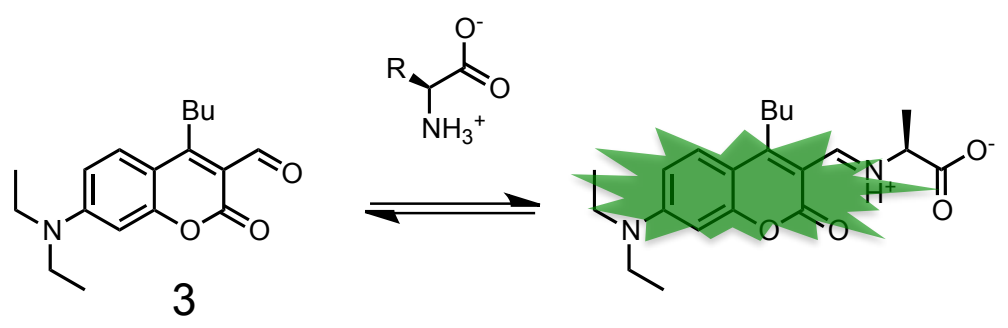

Figure 1.7: Formation of the iminium ion via condensation of a primary amine with a coumarin aldehyde. In this case the primary amino is provided by an amino acid.

The example of coumarin aldehydes binding to primary amines is an illustration of direct chemical modification of the D - $\pi$ - A system of the fluorophore. Because the signal outputs from the bound and unbound state can be clearly differentiated, it is an effective motif for amine binding and quantification. There are also other mechanisms of fluorescence changes that can be utilized. In work by Nagano's group, a Si-Rhodamine based sensor was utilized for NIR calcium sensing see Figure 1.8, [9].

This calcium sensor works through a photo-induced electron transfer (PET) mechanism, this means that in one state, which in this example is the state without calcium bound, a secondary conjugated system has HOMO and LUMO energy levels that align in such a way to inhibit radiative decay as a de-excitation pathway from the excited state. Figure 1.9 gives a graphical depiction of PET mechanisms, the work by Nagano's group is an example of A-PET.

Nagano's calcium sensor works through use of a BAPTA motif which is well characterized for calcium binding [10]. Calcium sensing itself has relevance due to the role of calcium in neuronal function [11]. 

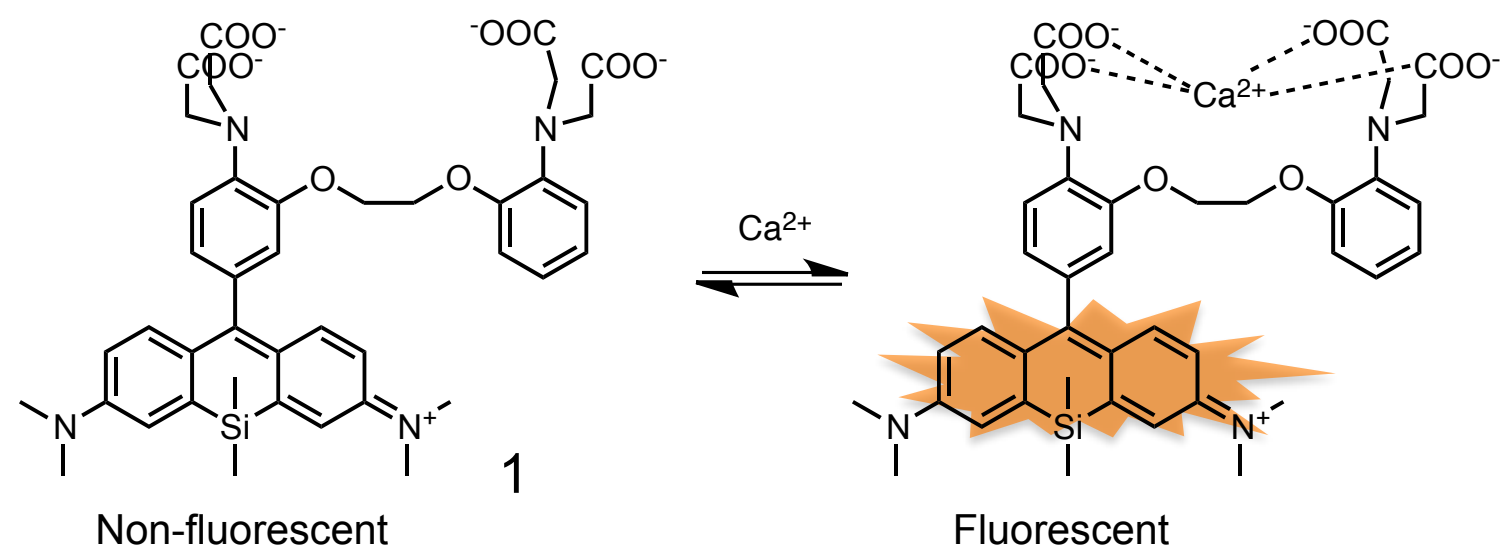

Fluorescent

Figure 1.8: : A NIR Si-Rhodamine with pendant BAPTA group, expresses little fluorescence on it's own, but in the presence of calcium, PET Quenching is relieved and fluorescence is turned "on".

Another mechanism through which fluorescence emission can be modulated is Förster Resonance Energy Transfer (FRET). FRET is the process by which upon de-excitation, instead of emitting a photon, the excited system transfers that energy to a second fluorophore, which then emits. The two involved fluorophores are referred to as a FRET pair. FRET has several contributing components, the two fluorophores must be localized together at a distance which allows this energy transfer to occur. Many FRET pair interactions are used to visualize binding interactions that modify large constructs. Binding events change cause the construct to chemically change, or structurally rearrange to change the distance between the two fluorophores, causing the FRET efficiency to change in a quantifiable way. More importantly for a FRET pair, there must be an overlap between the emission of the FRET donor, and the absorption of the FRET acceptor, see Figure 1.10 [12]. The amount of this overlap, combined with the discrete distance between the two fluorophores determines FRET efficiency. Changing either the distance involved, or the absorption or emission of either pair can allow for a change in the emission output which can be quantified. 

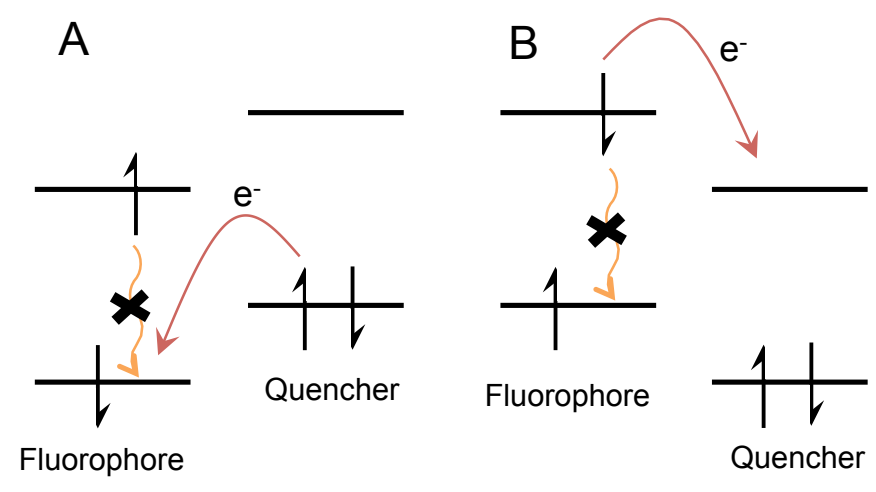

Figure 1.9: : A - Acceptor PET, where radiative decay from the excited state is blocked by electron transfer from a nearby HOMO, B - Donor PET, where radiative decay is a less favorable pathway of de-excitation because of a nearby LUMO that acts as an electron acceptor.

A cleverly conceived example of FRET can be illustrated in work by Chris Chang's group, where a Coumarin-Fluorescein FRET system was used to construct a Hydrogen Peroxide $\left(\mathrm{H}_{2} \mathrm{O}_{2}\right)$ probe, Figure 1.11. Chang's group constructed a coumarinprotected-fluorescein construct, with the fluorescein locked into the non-emissive spirolactone form via pinacol-borane protecting groups. Excitation of the coumarin with fluorescein still protected yields fluorescence from the coumarin. However, upon exposure to $\mathrm{H}_{2} \mathrm{O}_{2}$, the boronic acid groups are cleaved allowing the spirolactone to open, and the fluorescein to convert to a fluorescent form.

Methods of modulating fluorescence are diverse. As fluorescent sensors are environment sensitive, they are sensitive to changes in solvent polarity, and osmotic pressure along with their designed mode of analyte recognition. One of the many key requirements for fluorescent sensors, despite the mode of sensing, is that emission changes are quantifiable. This provides us with the two core pieces of a successful fluorescent sensor. It must have a change in emission signal due to a specific chemical event, and that signal must be measurable. 


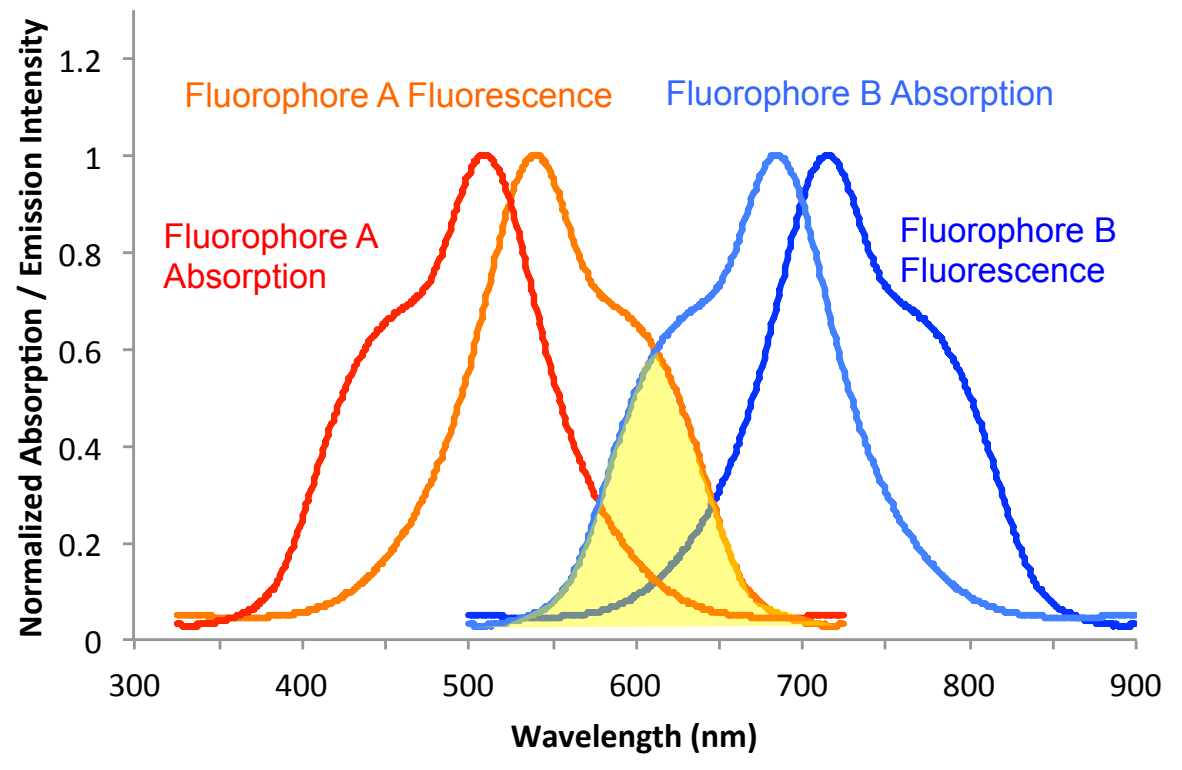

Figure 1.10: Fret relies on the overlapped energy levels of absorption and emission between tethered, or locally related fluorophores.

\subsubsection{Binding}

Fluorescent sensors, or any other type of sensor, must associate in some manner with a target analyte. Often, these interactions are supramolecular. Supramolecular interactions can be subcategorized into several types: ion pair interactions, ion-dipole interactions, dipole-dipole interactions, hydrogen bonding, cation- $\pi, \pi-\pi$, and hydrophobic interactions. All of these supramolecular interactions occur with varying strengths, see Figure 1.1 [13]. Sensors can also work through covalent chemistry, involving the forming and breaking of bonds as the structural mechanism for modulation of the change in fluorescence emission, or absorbance. Fluorescence is preferred over absorbance.

Work conducted by the Glass Group involving coumarin aldehydes relies on covalent chemistry to reversibly bind primary amines. Many dosimeters also rely on 


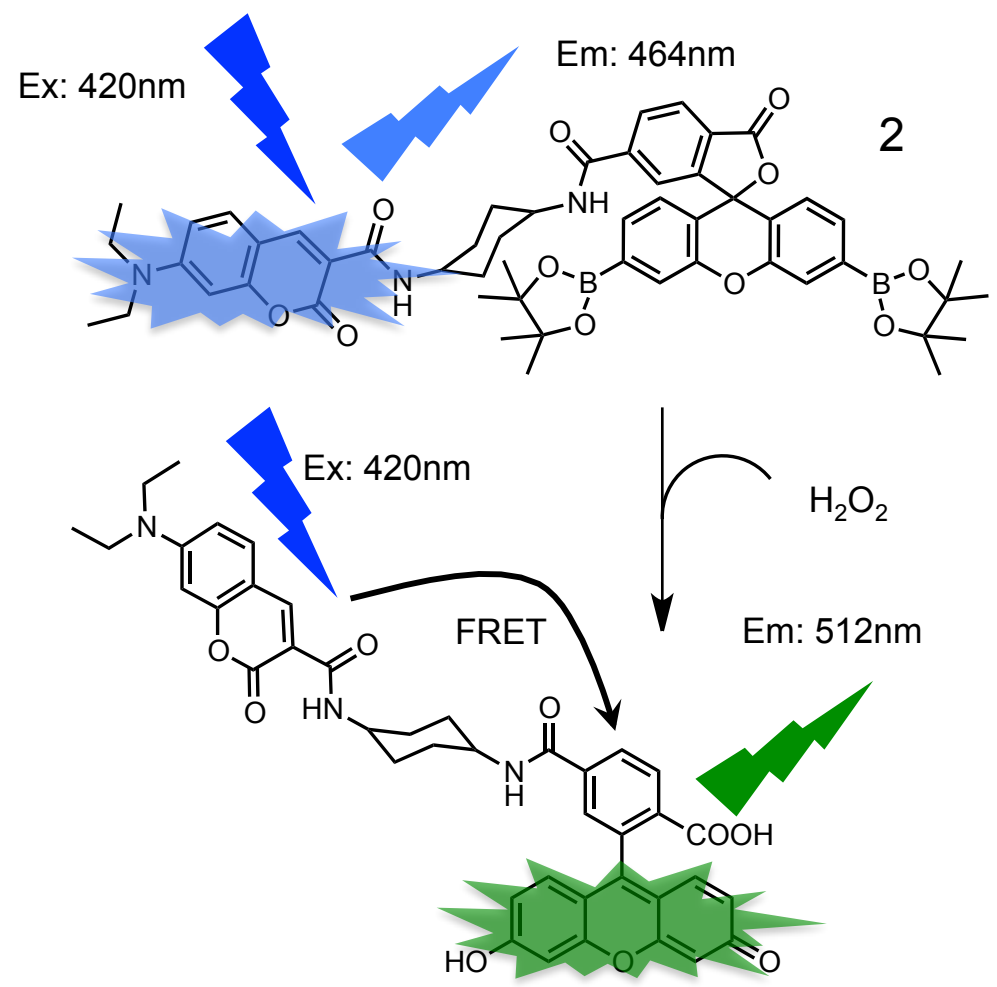

Figure 1.11: Before exposure to $\mathrm{H}_{2} \mathrm{O}_{2}$, excitation of the coumarin yields fluorescence from only the coumarin, but upon exposure to $\mathrm{H}_{2} \mathrm{O}_{2}$, and cleavage of the Pinacol Borane protecting groups, excitation of the coumarin yields fluorescence from the now deprotected fluorescein

covalent chemistry to produce an irreversible structural change that modulates fluorescence emission. Binding events cause changes in fluorescence or absorbance that can be described mathematically. The nature of the mathematical description of the data generated provides us with information about the binding event itself. The most common binding events in sensor chemistry involve 1:1, analyte:sensor stoichiometry, and are described mathematically with a one-site binding isotherm. Which is describe in Equation 1.1

$$
y=\frac{B_{\operatorname{Max}} \times x}{K_{d}+x}
$$




\begin{tabular}{|c|c|c|}
\hline Interaction Type & Strength (in KJ/mol) & Example \\
\hline Ion - Ion & $200-300$ & Acetate - Ammonium \\
\hline Ion - Dipole & $50-200$ & Crown ether - Cation \\
\hline Dipole - Dipole & $5-50$ & $\begin{array}{c}\text { Hemoglobin subunit } \\
\text { interactions }\end{array}$ \\
\hline Hydrogen Bonding & $4-120$ & DNA Base Pair interactions \\
\hline Cation $-\pi$ & $5-80$ & Cation solvation in Benzene \\
\hline$\pi-\pi$ & $0-50$ & Graphite \\
\hline Hydrophobic Interactions & Relative to solvent & Buckycatcher ${ }^{1}$ \\
\hline
\end{tabular}

Table 1.1: Table of Interatction Forces [14].

The one-site binding isotherm mathematically provides descriptions for both concentration of analyte that fully saturates the sensor, and the concentration of analyte that provides half saturation of analyte. The half saturation is alternatively described as the Dissociation Constant $\left(\mathrm{K}_{\mathrm{d}}\right)$ or Association Constant $\left(\mathrm{K}_{\mathrm{a}}\right)$. The divergence in terminology arises from different fields of study preferring to describe their data in different ways.

$\mathrm{K}_{\mathrm{d}}$ describes half saturation as a molar concentration, and uses $\mathrm{M}$ as units, while $\mathrm{K}_{\mathrm{a}}$ describes half saturation as inverse molar, using $\mathrm{M}^{-1}$ units. Both $\mathrm{K}_{\mathrm{d}}$ and $\mathrm{K}_{\mathrm{a}}$ provide measures of binding strength by denoting the half saturation point, Figure $1.12[15]$.

Another common binding mode observed in sensor chemistry is a sigmoidal fit. Sigmoidal fits are often recognizable from $\mathrm{pK}_{\mathrm{a}}$ titrations, or $\mathrm{EC}_{50}$ dose response experiments. In both cases, a log scale is used to describe concentration, while intensity is measured in absorbance or fluorescence. The general form of a sigmoid is described in Equation 1.2 [15]. 


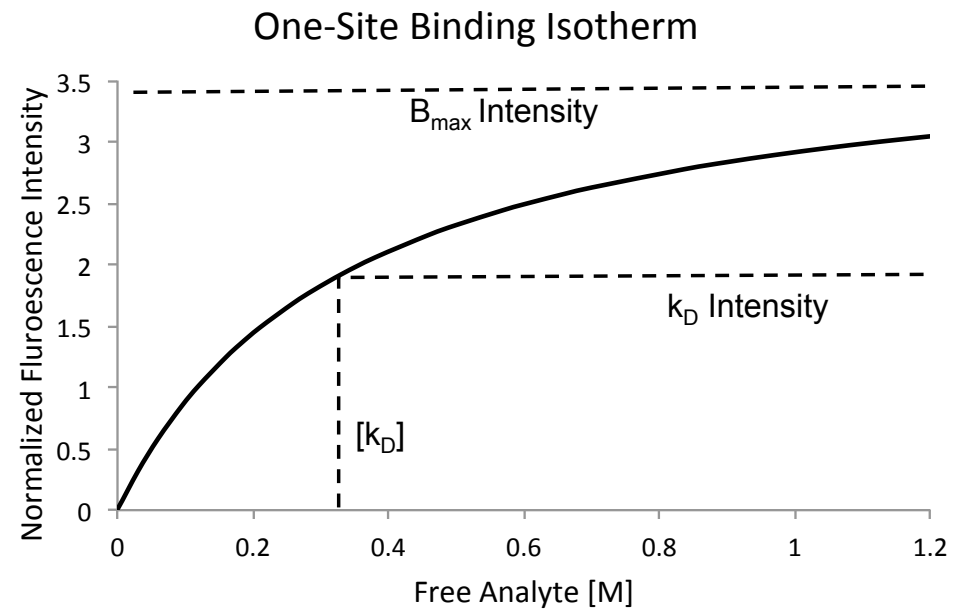

Figure 1.12: : A generic right hyberbola depicting the curve of a one site binding equation. The $\mathrm{K}_{\mathrm{d}}$, and $\mathrm{B}_{\mathrm{Max}}$ are labeled.

$$
y=\text { Minimum }+\frac{\text { Maximum }- \text { Minimum }}{1+\frac{x}{\text { InflectionPoint }}^{- \text {HillCoefficient }}}
$$

The maximum and minimum values describe limits of intensity measures by total saturation, or complete lack of binding, respectively. The hill coefficient is a description of cooperativity, or how individual binding events positively, or negatively effect other binding events. The inflection point translates to a $\mathrm{pK}_{\mathrm{a}}$ value, or $\mathrm{EC}_{50}$ value, an effective halfway value between the min and max, informed by the cooperativity inherent in the signal producing events being investigated.

$$
y=F L I n t_{\text {Min }}+\frac{F \operatorname{LInt}_{\text {Min }}-F \text { LInt }_{\text {Min }}}{1+\frac{x}{p K_{a}}-\text { HillCoefficient }}
$$

Equation 1.3 produces a sigmoidal curve as shown in Figure 1.13. When $\mathrm{pK}_{\mathrm{a}} \mathrm{s}$ are determined experimentally, the terms of Equation 1.2 are revised to provide Equation 1.3. Mathematically the same, the change of terms is helpful as the 'analyte' in this 


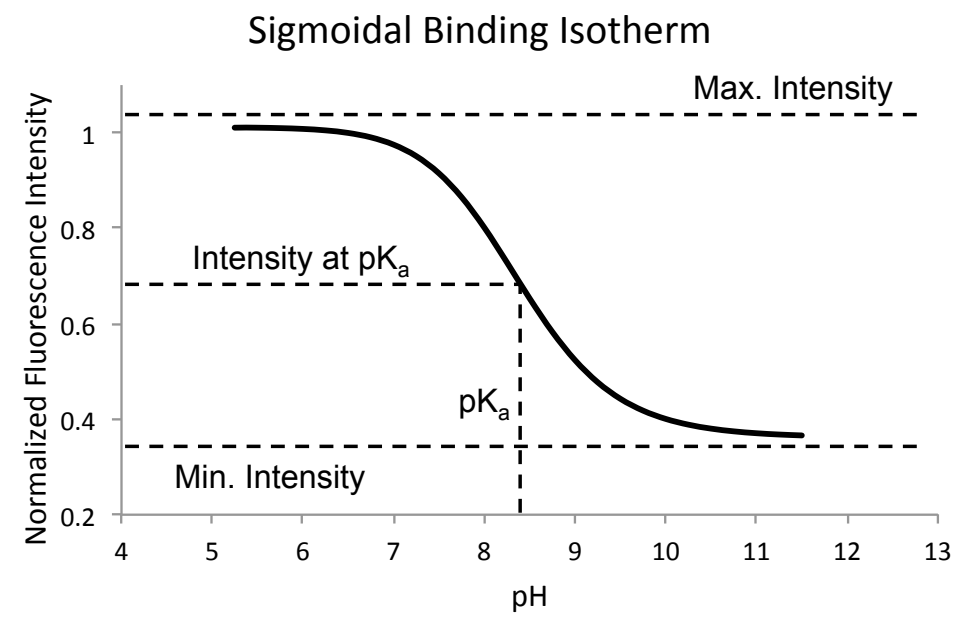

Figure 1.13: : A sigmoidal curve representing a generic $\mathrm{pK}_{\mathrm{a}}$ determined experimentally through fluorescence measurements.

case, a proton, is measured in concentration on a log scale.

Successful design of a sensor relies on tuning the binding characteristics such that changes in signal outputs can be achieved at changes in concentration of the target analyte that are relevant. A sensor that is designed for use in vivo, but is fully saturated at biologically relevant concentrations of it's target analyte will provide no useful changes in signal output. Similarly, if binding is too weak, or if the sensor binds competitively with compounds besides the target analyte, there will be confounding signal outputs rendering the designed sensor unusable.

\subsubsection{Biologically Relevant Analytes}

The number of molecular species for which to design chemical sensors are manifold. There is significant interest in monitoring specific ions in cells, such as zinc, calcium, sodium and potassium, as many are used in neuronal signaling or in signal cascades 
that trigger cell functions. Some ions, like zinc, copper, and iron are plentiful in the cell, but tightly bound in proteins and rarely free in solution. Dis-regulation of ions whose concentrations are tightly controlled can lead to disease states, or failure of cell functions to be triggered appropriately. Small molecules and proteins also garner interest, neurotransmitters and neurotoxins whose activities can be accurately described and quantified can be more clearly understood.

\subsubsection{Sugars}

Carbohydrates provide fertile targets for chemical sensors. Glucose metabolism disregulation is the lynchpin of diabetes mellitus in humans and other mammals. Accurate measures of blood glucose are currently simple, but are invasive, creating problems with monitoring fidelity. Protein glycosylation patterns help cells communicate, and can be targeted by antibiotics. Glycosylation motifs of glycolipids have been implicated as potential cancer therapeutic targets. Blood types are also determined by glycosylation patterns, with the A and B blood antigens differing by a single portion of a pentasaccharide. Fluorescent chemical sensors that can quickly provide information about the distribution or disruption of any of these analytes provide potential value in therapeutics and diagnostics.

\subsubsection{Aggregation}

Most fluorescent sensors, and fluorescent compounds in general are built on rigid, aromatic scaffolds. This general design motif, necessary for desired emission and absorption characteristics creates some drawbacks for compounds designed for use in 
aqueous systems, as rigid organic structures can often lack solubility in polar protic environments. Even when these systems are soluble, they can aggregate in solution. Changes from monomer to aggregate states can cause changes in fluorescence, which if unaccounted for can confound output signals in fluorescent sensors. Aggregation is a well studied phenomenon, and not limited to synthetic compounds; the molecular basis for Alzheimer's Disease is an aggregation of Amyloid- $\beta[16,17]$ proteins, leading to dysfunction in neurons.

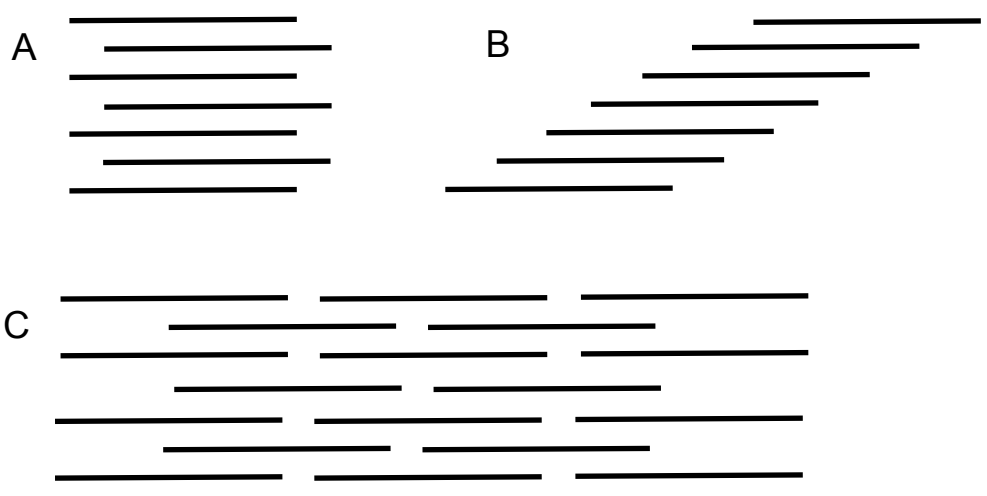

Figure 1.14: : A - Ladder type, B - Staircase type, C - Brickwork type

Formation of aggregates by compounds being used as fluorescent sensors confounds both the supramolecular interactions that drive binding, and the emission output being used as a signal. Aggregation affects to binding events will be specific to both the type of aggregate, and the individual binding event, but aggregation affects on emission are well understood and classified into either J-aggregates or H-aggregates. J-aggregates are named after E. E. Jelley who first observed their formation in Pseudoisocyanine (PIC), while H-aggregates are named after their hypsochromic effect on the absorption. Aggregates themselves are proposed to form one-dimensional assemblies in solution, Figure 1.14 [18, 19, 20].

Aggregation is divided into H- and J- types because of the different ways that ab- 


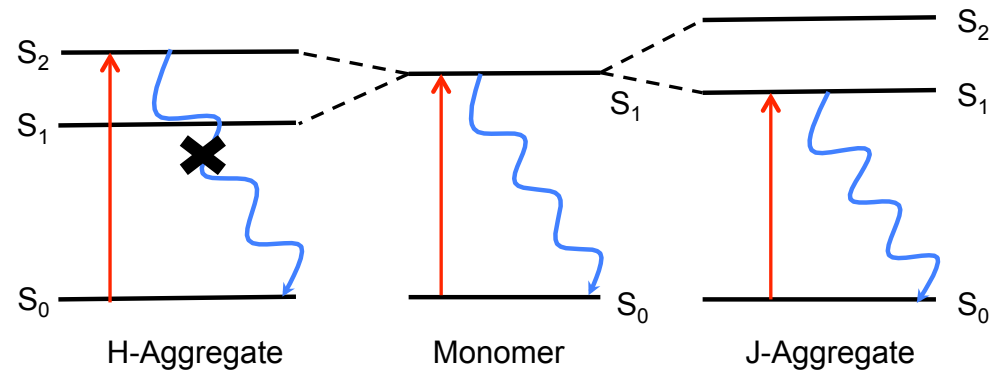

Figure 1.15: In $\mathrm{H}$-aggregates emission from an $\mathrm{S}_{2}$ state is non-emissive, while in J-aggregates emission from an $\mathrm{S}_{1}$ state is highly favored.

sorbance and fluorescence are affected. J-aggregates present a red shifted absorbance, and an increase in fluorescence intensity, while H-aggregates present a blue shifted absorbance and a loss of fluorescence intensity [21]. This is related to how the aggregates themselves form, and how the Acceptor - $\pi$ - Donor system is affected by the electronics of these environmental changes. Aggregation behavior can be described through molecular exciton theory, aggregate formation causes excited state energy levels to split. In cases where excitation to the higher energy split is allowed or favored, fluorescence becomes disfavored. This is related to Kasha's Rule, which states that fluorescence occurs from the lowest energy excited state [22]. In cases where excitation to the lower energy split level is favored, fluorescence is retained, and intensity increases compared to the monomer Figure 1.15 [23]. 


\section{Chapter 2}

\section{pH Sensing with Tetra Sulfonated Cyanine Compounds}

\section{$2.1 \mathrm{pH}$ in vivo}

Proton recognition and trafficking is integral to cellular health and function $[24,25$, 26]. Proton gradients across membranes can be used as the driving force for reactions in cells, and localized $\mathrm{pH}$ can be used to favor or disfavor metabolic and catabolic pathways, or intracellular processes [27, 28]. Because of this, it is advantageous to monitor regulation in physiological $\mathrm{pH}$. Insight into disregulation of $\mathrm{pH}$, or designed deviations in $\mathrm{pH}$ can provide insight into the cellular functions and processes that protonation states can affect.

Both intracellular and extracellular $\mathrm{pH}$ in humans is regulated tightly, with extracellular pH kept regulated around 7.4, while intracellular pH vary depending largely on cell type. Deviations in intracellular $\mathrm{pH}$ can be caused by disease states such as 
cancer, alzheimers or by disfunction in metabolic pathways $[29,30]$. One consequence of this is that extracellular $\mathrm{pH}$ in blood vessels can vary by location in the body, such as proximity to specific organs, or potentially a tumor [29]. A fluorescent $\mathrm{pH}$ sensor that circulates in the blood stream could potentially provide a minimally invasive means of monitoring metabolic changes which could lead to local or global changes in $\mathrm{pH}$. To achieve this end, this potential sensor would need to be water soluble. One of the central features of working with blood, its distinctive red color, comes from hemoglobin, which absorbs far into the red region of the visible spectrum. Any fluorescent sensor designed to work in vivo, in any close proximity to hemoglobin, must be excited, and emit at wavelengths beyond Hemoglobin absorption [31]. This potential $\mathrm{pH}$ sensor would therefore have to have excitation and emission bands in the near-infrared (NIR).

\subsection{1 pH Sensing in The Literature}

The standard for determining cytoplasmic $\mathrm{pH}$ in vivo is BCECF 4, a stable water soluble fluorescein derivative, 2.1 [32]. First published by Roger Tsien's group in 1982, BCECF was the beginning of a deluge of modified fluorescein compounds for use as fluorescent indicators [33].

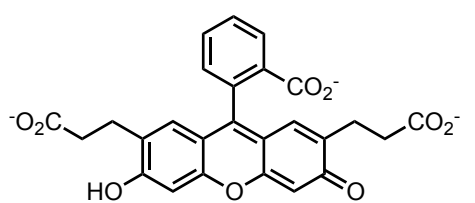

4

Figure 2.1: 2',7'-Bis-(2-carboxyethyl-5-(and-6-)carboxyfluorescein, (BCECF) 


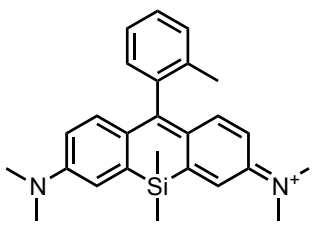

5

Figure 2.2: Simple Si-Rhodamine

Fluorescein, and similarly structured Rhodamine derivatives provide many significant advantages; they are chemically stable and photo-stable, and provide advantageous photo-physical properties for ratiometric fluorescent sensing [34]. The largest disadvantage of this family is that excitation wavelengths often lie below $600 \mathrm{~nm}$, which is disadvantageous for work in vivo [31]. Attempts to address this relatively low excitation wavelength have been made using Si-Rhodamine cores such as $\mathbf{5}$, which have shown some promise, but are currently synthetically arduous to access, Figure $2.2[35]$.
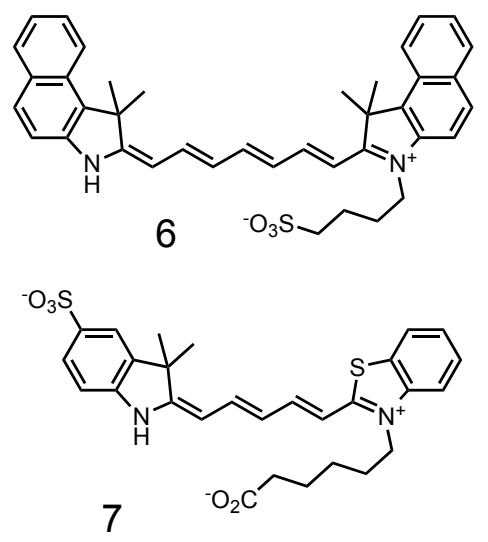

Figure 2.3: $\mathrm{pH}$ sensitive unsymmetrical cyanines, utilizing an un-alkylated indole.

In an attempt to perform $\mathrm{pH}$ sensing using higher excitation wavelengths, cyanine derivatives have been pursued [36, 37, 38]. Though providing higher excitation and 
emission wavelengths, cyanines often show poor stability, easily photo-bleach, and have poor quantum yields $[39,18]$. Unsymmetrical cyanines, with an un-alkylated indole present on one side have shown near-neutral $\mathrm{pK}_{\mathrm{a}} \mathrm{s}$, providing fluorescence increases upon protonation, Figure $2.3[40,41]$.

Additionally PET based pH sensors have been developed using cyanine scaffolds, where amine substituents are substituted for the meso-chloride, $2.4[36,42]$. The free lone pair on the amine functions as a PET quencher, and upon protonation, quenching is relieved, turning fluorescence on.

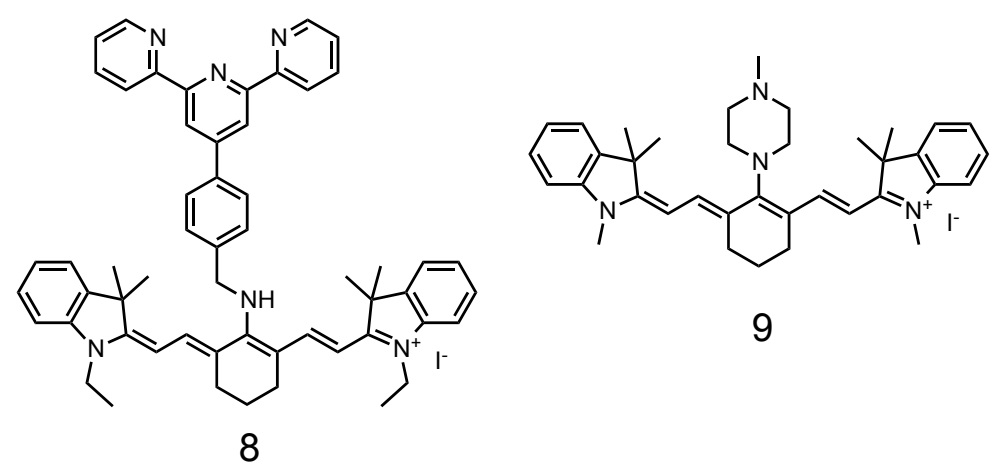

Figure 2.4: $\mathrm{pH}$ sensitive cyanines that use an amine lone pair as a PET mechanism for fluorescence quenching.

\subsubsection{Tetra-sulfonated Amino-Cyanine $\mathrm{pH}$ Sensors}

This project was originally pursued using IR- 820 derivates, and though some success was found, it was eventually determined that the compounds described were unsuitable for use in fully aqueous systems due to aggregation effects [43]. The central IR-820-piperazine adduct described in that work has recently been published by a separate group [38]. Therefore a tetra-sulfonated cyanine core was chosen as the basis to continue this project, in part because a substantial body of literature exists 
on performing chemistry on the meso-chloride of many commercially available cyanines. It was hypothesized that the addition of two extra sulfonate functional groups, specifically on the napthindole rings of the cyanine, would help to alleviate aggregation effects. Also, with a goal of performing studies in vivo it is important to note that cyanine dyes have been utilized safely in humans since Indocyanine Green (ICG) was first approved by the FDA in 1959. Though individual compounds will differ in efficacy in vivo, designing a sensor for use in humans based on scaffolds already approved by the FDA seems prudent.

The tetra-sulfonated core, compound $\mathbf{1 0}$ originally proved resistant to substitution chemistry at the chloride position. This was eventually circumvented through the use of a palladium catalyst. To date with this project it should be noted that no modification of the chloride has been achieved without palladium present, and to some extend the starting material is resistant to decomposition in the absence of palladium. This is somewhat unusual for a cyanine dye. Though favored for their optical properties, cyanines are known to oxidatively decompose relatively easily. An interesting aside in terms of reactivity has been observed with the tetra-sulfonated indole based cyanine, which is amenable to substitution chemistry without transition metal catalysis.
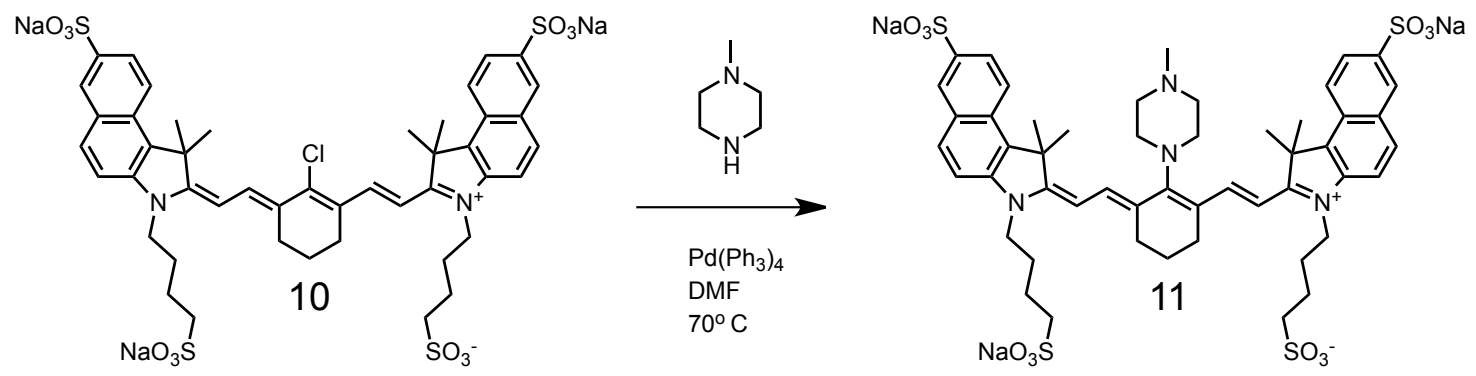

Figure 2.5: Synthesis of Methyl-Piperazine appended cyanine, Compound 11 
Compound 11, chosen as an initial starting point, was synthesized in reasonable yield, and isolated by preparative scale HPLC. Initial $\mathrm{pH}$ titration experiments revealed a single dominant absorption band at basic $\mathrm{pH}$, which is replaced by two red shifted absorption bands at acid $\mathrm{pH}$.

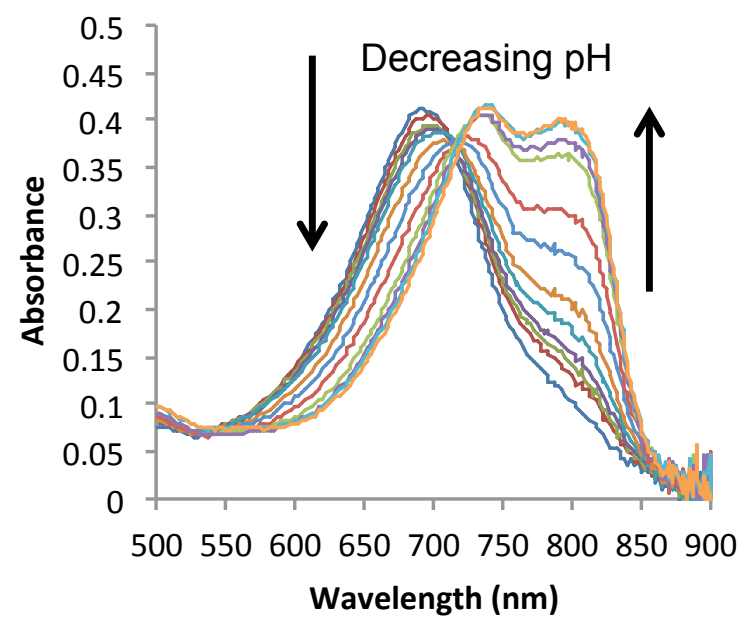

Figure 2.6: Compound $\mathbf{1 1} \mathrm{pH}$ titration by absorbance spectroscopy, $10 \mu \mathrm{M} .165 \mathrm{mM}$ PBS, $\mathrm{pH}$ adjustments with $1 \mathrm{M} \mathrm{HCl}$.

When followed by fluorescence and exciting at $670 \mathrm{~nm}$, compound 11 provides a $\mathrm{pK}_{\mathrm{a}}$ of 6.9 , when fitting fluorescence intensity at $820 \mathrm{~nm}$. Which is advantageous for monitoring $\mathrm{pH}$ changes in vivo. Because prior work on these compounds indicated that cyanine derivates can present aggregation issues in aqueous systems, titration experiments were repeated at various concentrations.

The consistency of the data collected from exciting at $670 \mathrm{~nm}$ and observing fluorescence intensity at $820 \mathrm{~nm}$ was not reflected in other excitations, and intensity observations. For instance, when exciting at $740 \mathrm{~nm}$, near what appears to be an isosbestic point in the absorbance spectra, fluorescence intensity observations, regardless 

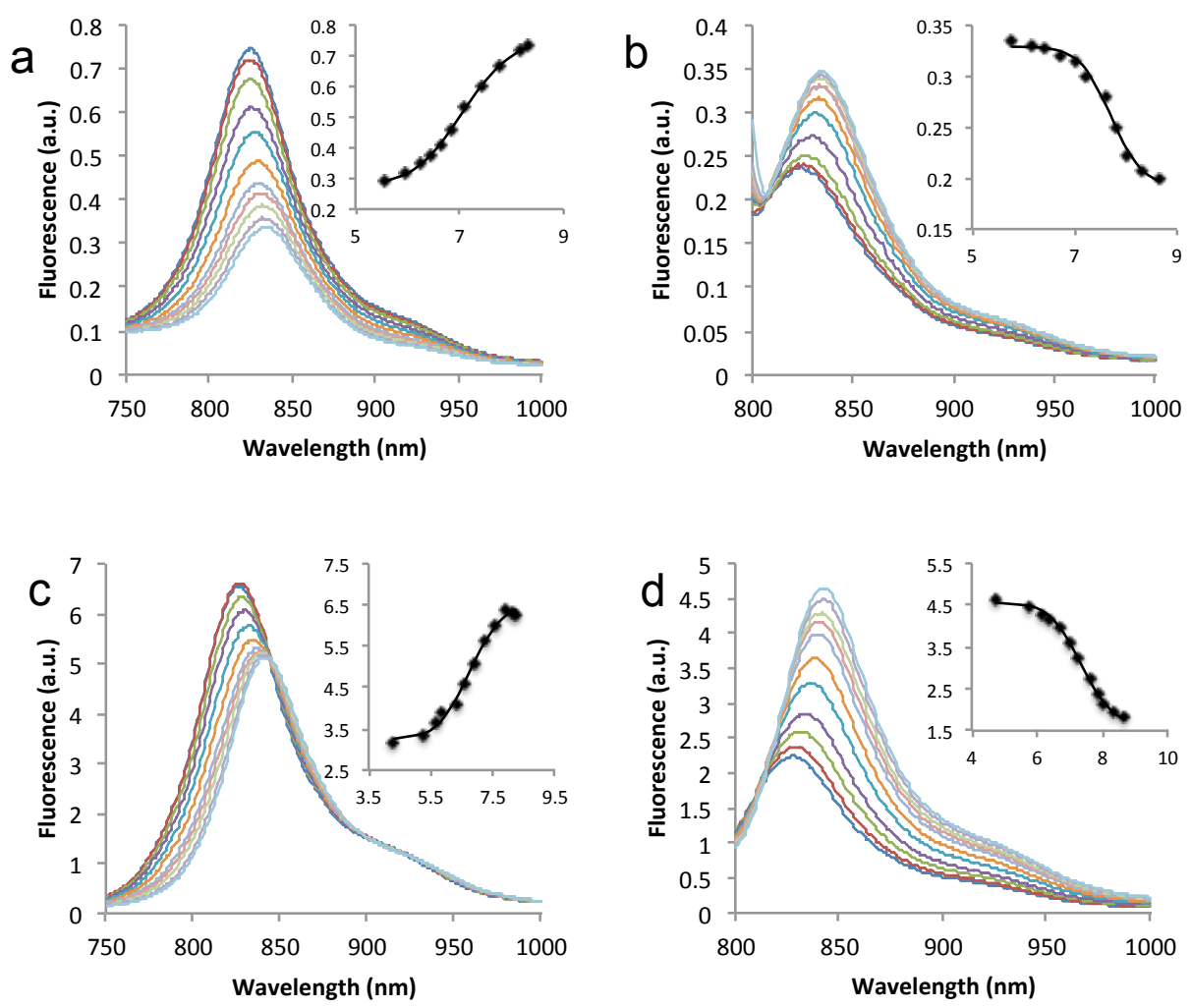

Figure 2.7: Compound $11 \mathrm{pH}$ titration by fluorescence spectroscopy $2.5 \mu \mathrm{M}(\mathrm{a}, \mathrm{b})$ and $20 \mu \mathrm{M}(\mathrm{c}, \mathrm{d}) .165 \mathrm{mM}$ PBS, pH adjustments with $1 \mathrm{M} \mathrm{HCl}$. Slit widths were $15 \mathrm{~nm}$. Excitation at $670 \mathrm{~nm}$ (a) and $780 \mathrm{~nm}$ (b), insets are intensity at $820 \mathrm{~nm}$ (a,c) and 840nm (b,d) plotted against $\mathrm{pH}$.

of location do not provide consistent $\mathrm{pK}_{\mathrm{a}}$ fits across all concentrations where titrations were performed. In some cases, fluorescence intensity observations do not even provide fit-able data. However for excitations performed at 780nm, while observing at $840 \mathrm{~nm}$, a modest trend emerges of an increasing $\mathrm{pK}_{\mathrm{a}}$ as concentration increases.

Observation of fluorescence as a whole shows that even for excitations at $670 \mathrm{~nm}$, which provide consistent $\mathrm{pK}_{\mathrm{a}} \mathrm{s}$ when specific intensity wavelengths are observed, emission profiles change drastically as concentration changes. This implies aggregation, however neither the fluorescence nor absorbance behavior is completely consistent 


\begin{tabular}{|c|c|c|c|c|c|}
\hline \multicolumn{6}{|c|}{$\mathrm{pK}_{\mathrm{a}}$ by Concentration for 11} \\
\hline & $1 \mu \mathrm{M}$ & $2.5 \mu \mathrm{M}$ & $5 \mu \mathrm{M}$ & $10 \mu \mathrm{M}$ & $20 \mu \mathrm{M}$ \\
\hline $\begin{array}{c}\text { Ex: 670nm } \\
\text { Em: 820nm }\end{array}$ & $\begin{array}{c}7.0 \\
(+/-0.1)\end{array}$ & $7.0(+/-0.1)$ & $6.8(+/-0.2)$ & $7.0(+/-0.1)$ & $6.9(+/-0.2)$ \\
\hline $\begin{array}{c}\text { Ex: 780nm } \\
\text { Em: 840nm }\end{array}$ & & $7.2(+/-0.1)$ & $7.1(+/-0.2)$ & $7.3(+/-0.1)$ & $7.6(+/-0.1)$ \\
\hline
\end{tabular}

Table 2.1: $\mathrm{pK}_{\mathrm{a}}$ of $\mathbf{1 1}$ by Concentration

with either H- or J- aggregation effects. Also, given that the compound contains 4 sulfonate groups, which would remain deprotonated at the lowest pHs used in titration experiments, it seems difficult to invoke aggregation of such a charged molecule. The observed fluorescence and absorbance changes could be a result of $\mathrm{pH}$ induced aggregation becoming more favored as concentration increases, but the data collected does not allow for that to be determined with significant certainty. Aggregation affects would provide a convenient explanation for the second absorbance band observed in acidic conditions.
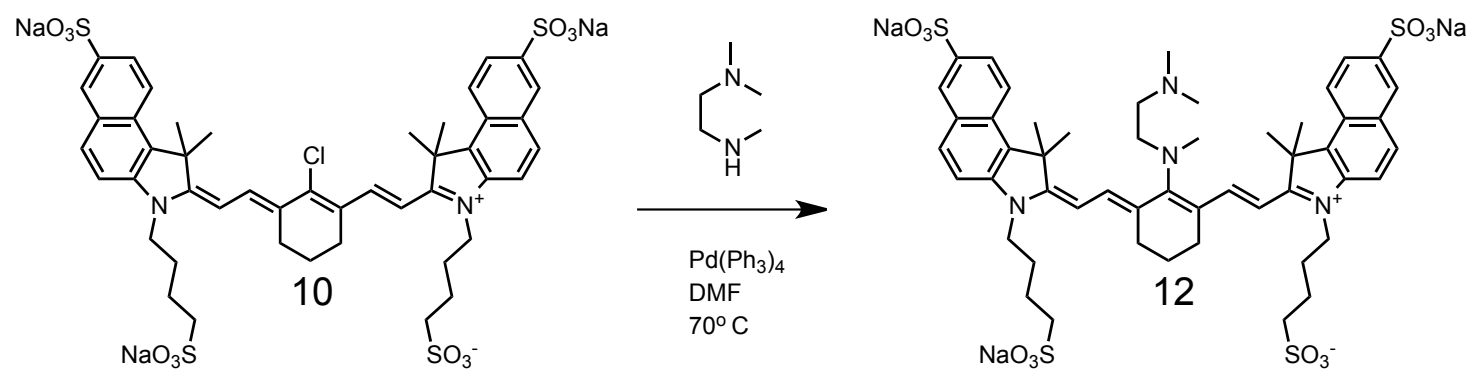

Figure 2.8: Synthesis of trimethylethylenediamine appended cyanine, Compound 12

Sensor 12 was pursued to determine how the distal nitrogen affects the $\mathrm{pK}_{\mathrm{a}}$ of the sensor overall. In previously published work, it was determined that having the second nitrogen attached through a chain, instead of being included in a ring, causes the $\mathrm{pK}_{\mathrm{a}}$ of the sensor to increase. That trend was found to continue with this analogue. Unlike 
Sensor 11, Sensor 12 did not show deviations between in observed $\mathrm{pK}_{\mathrm{a}}$ from separate excitation wavelengths.
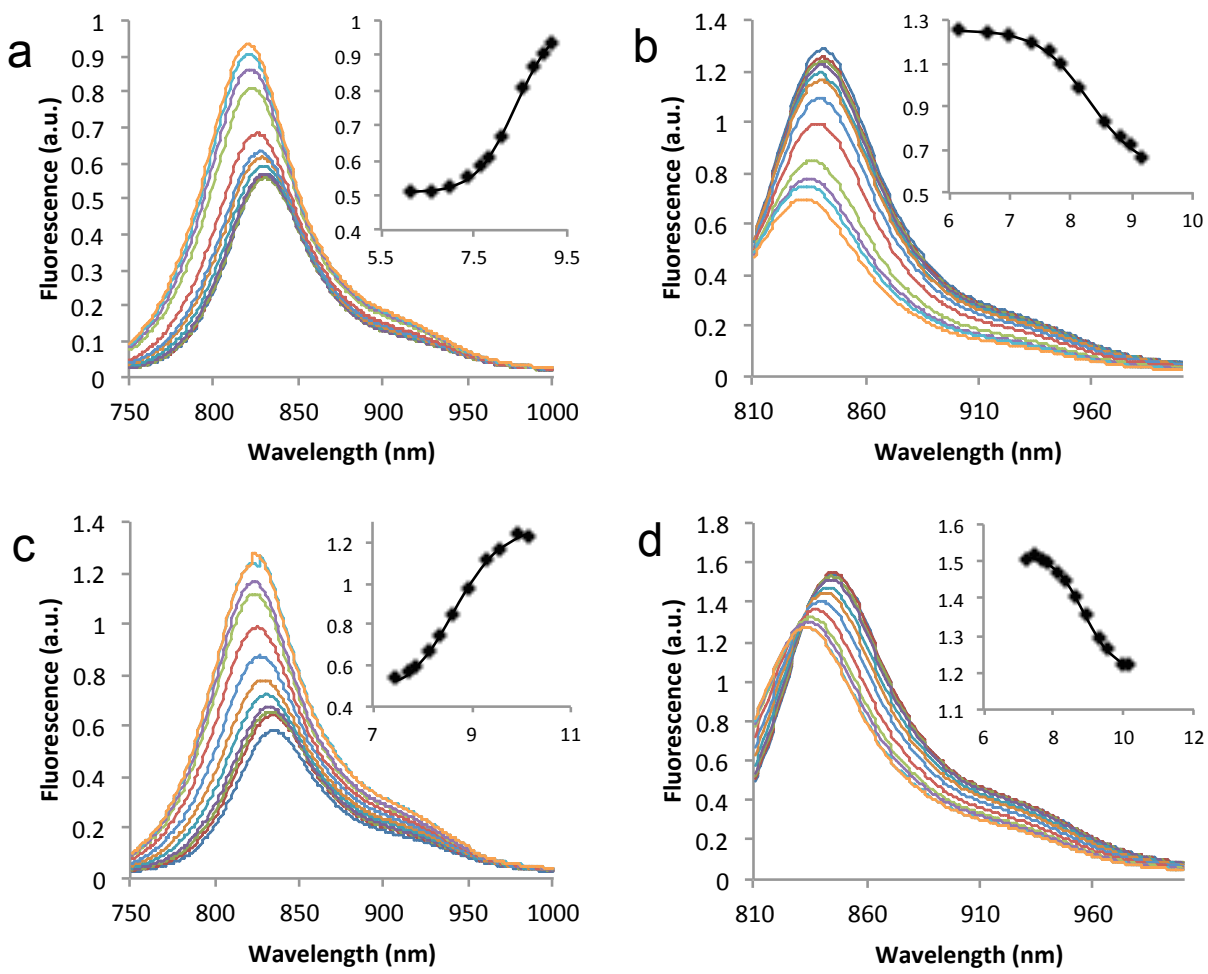

Figure 2.9: Sensor $12 \mathrm{pH}$ titration by fluorescence spectroscopy $2.5 \mu \mathrm{M}(\mathrm{a}, \mathrm{b})$ and $20 \mu \mathrm{M}(\mathrm{c}, \mathrm{d}) .165 \mathrm{mM}$ PBS, pH adjustments with $1 \mathrm{M} \mathrm{HCl}$. Slit widths were $15 \mathrm{~nm}$. Excitation at $670 \mathrm{~nm}$ (a) and $780 \mathrm{~nm}$ (b), insets are intensity at $820 \mathrm{~nm}(\mathrm{a}, \mathrm{c})$ and 840nm (b,d) plotted against $\mathrm{pH}$.

Sensor 12, when titrated against changing $\mathrm{pH}$, and observed by absorbance provided a profile that differed more than expected from 11. Although it should be expected that absorbance profiles would be unique, the dissimilarity between the absorbance maxima of the two compounds at their most acid is interesting. When interrogated via fluorescence, 12 maintained a constant $\mathrm{pK}_{\mathrm{a}}$ of 8.1 across three of the concentrations chosen. However at the highest concentration, $20 \mu \mathrm{M}$, that consistent 
$\mathrm{pK}_{\mathrm{a}}$ is lost and $\mathbf{1 2}$ provides a $\mathrm{pK}_{\mathrm{a}}$ of 8.9 .

\begin{tabular}{|c|c|c|c|c|}
\hline \multicolumn{5}{|c|}{$\mathrm{pK}_{\mathrm{a}}$ by Concentration for 12} \\
\hline & $2.5 \mu \mathrm{M}$ & $5 \mu \mathrm{M}$ & $10 \mu \mathrm{M}$ & $20 \mu \mathrm{M}$ \\
\hline $\begin{array}{c}\text { Ex: 670nm } \\
\text { Em: 820nm }\end{array}$ & $8.4(+/-0.1)$ & $8.3(+/-0.2)$ & $8.5(+/-0.1)$ & 8.9 \\
\hline $\begin{array}{c}\text { Ex: 780nm } \\
\text { Em: 840nm }\end{array}$ & $8.4(+/-0.1)$ & $8.4(+/-0.2)$ & $8.5(+/-0.1)$ & 8.9 \\
\hline
\end{tabular}

Table 2.2: $\mathrm{pK}_{\mathrm{a}}$ of $\mathbf{1 2}$ by Concentration

The next compound in the series, $\mathbf{1 3}$ was chosen to determine if the system would still function as a $\mathrm{pK}_{\mathrm{a}}$ sensor without the distal nitrogen. This derivative would probe whether the distal nitrogen is necessary for a fluorescence response, or only provides a change in $\mathrm{pK}_{\mathrm{a}}$.

Though fluorescence emission changes were observed as $\mathrm{pH}$ was modulated, $\mathbf{1 3}$ did not provide intensity data that could be fit to determine a $\mathrm{pK}_{\mathrm{a}}$, over the $\mathrm{pH}$ ranges tested for Sensor 11 and Sensor 12. Given that a vinyl amine would potentially present a $\mathrm{pK}_{\mathrm{a}}$ similar to that of an aniline, titrations were attempted under more acidic conditions, but $\mathbf{1 3}$ proved to be too unstable under $\mathrm{pH} 4$ to perform such titrations and gain an accurate $\mathrm{pK}_{\mathrm{a}}$.
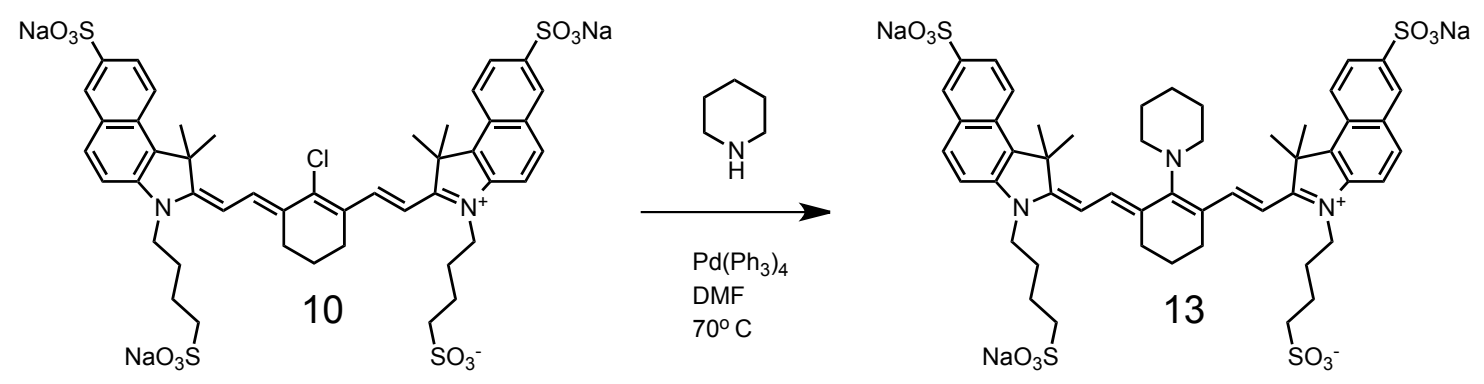

Figure 2.10: Synthesis of Piperidine appended cyanine, Compound 4

Sensor 14 was pursued with the intention of further exploring the nature of the 
$\mathrm{pH}$ induced change in fluorescence. In 11 and 12 two mechanisms can be proposed as causing $\mathrm{pH}$ associated fluorescence emission changes; either the lone pair of the nitrogen attached directly to the $\pi$ system becomes tied up in an $\mathrm{N}-\mathrm{H}$ bond, causing fluorescence modulation through Internal Charge Transfer, or the lone pair of the distal nitrogen becomes tied up in an N-H bond and modulates fluorescence through the relief of PET quenching. It is assumed that if PET is the responsible mechanism, 14 would provide a similar $\mathrm{pK}_{\mathrm{a}}$, and fluorescence behavior to $\mathbf{2}$.
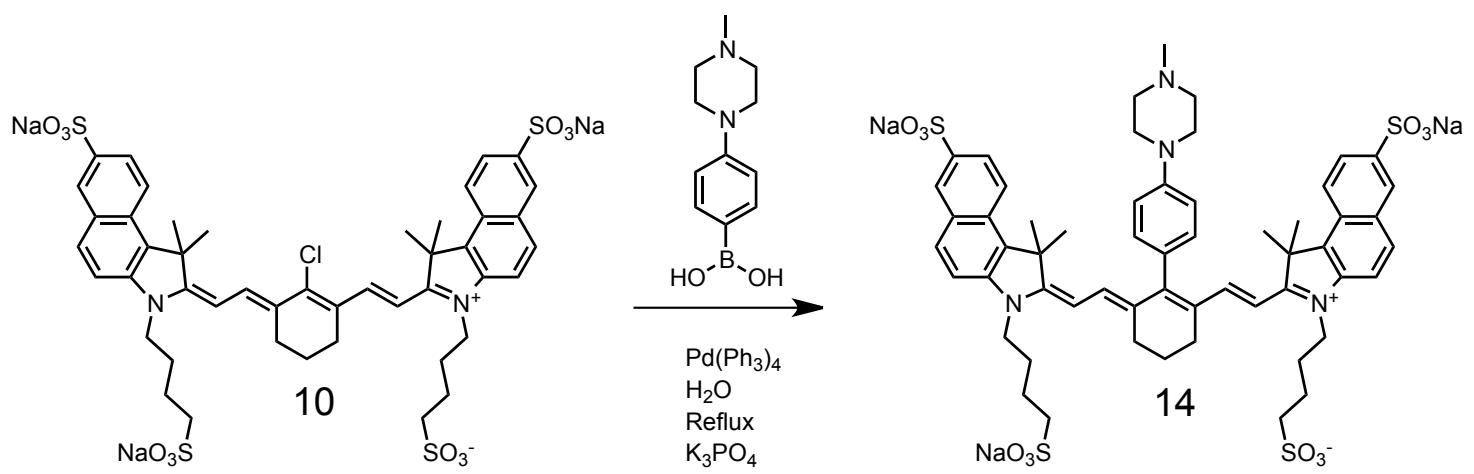

Figure 2.11: Synthesis of phenyl-methylpiperazine appended cyanine, Compound 5

Interestingly, though qualitative comparison of emission spectras alone may give the indication that 14 supports this hypothesis, analysis of emission intensity presents data that complicates the overall picture of the mechanism of fluorescence modulation. Whereas 11 and 12 present data that gives only some indication of aggregation at higher concentrations, 14 provides fluorescence behavior that indicates aggregation. Again the overall absorption and emission behavior does not fully match that described for $\mathrm{H}$ - or $\mathrm{J}$ - type aggregates, but a clear trend of an increasing $\mathrm{pK}_{\mathrm{a}}$ as the concentration of sensor is increased is best described by non-monomer behavior.

Sensor 14 presents further complicated fluorescence behavior versus concentration, in that exciting the absorbance band at $720 \mathrm{~nm}$, data that fits appropriately to a 

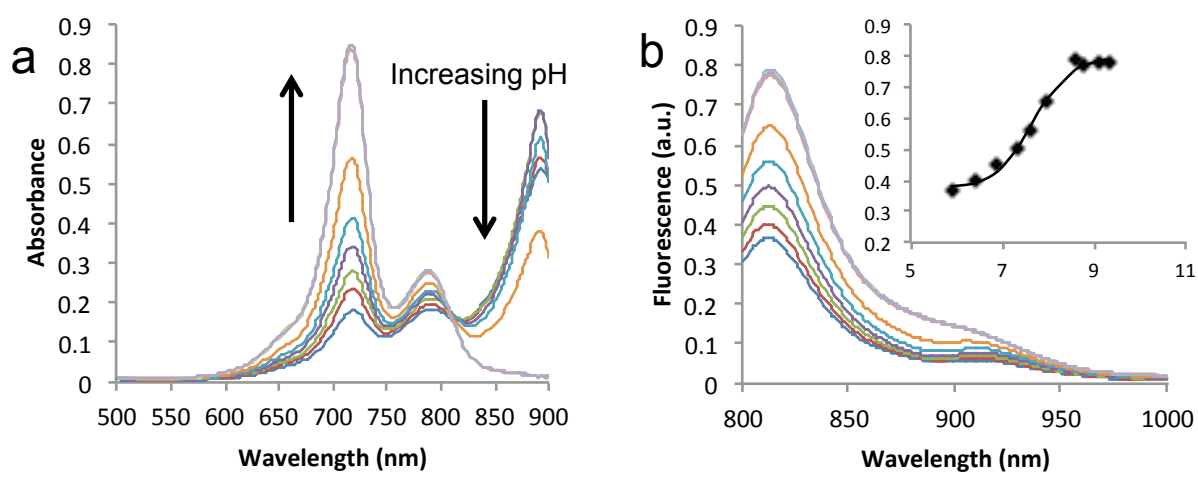

Figure 2.12: Absorbance (a) and Fluorescence (b) $\mathrm{pH}$ Titration of $\mathbf{1 4}$ at $5 \mu \mathrm{M}$, inset is fluorescence intensity at $810 \mathrm{~nm}$ plotted against $\mathrm{pH}$

\begin{tabular}{|c|c|c|c|c|}
\hline \multicolumn{5}{|c|}{$\mathrm{pK}_{\mathrm{a}}$ by Concentration for 14} \\
\hline & $2.5 \mu \mathrm{M}$ & $5 \mu \mathrm{M}$ & $10 \mu \mathrm{M}$ & $20 \mu \mathrm{M}$ \\
\hline $\begin{array}{c}\text { Ex: 770nm } \\
\text { Em: 810nm }\end{array}$ & - & $7.8(+/-0.1)$ & $8.1(+/-0.1)$ & $8.5(+/-0.1)$ \\
\hline $\begin{array}{c}\text { Ex:720nm } \\
\text { Em:810nm }\end{array}$ & - & $7.5(+/-0.2)$ & - & - \\
\hline
\end{tabular}

Table 2.3: $\mathrm{pK}_{\mathrm{a}}$ of $\mathbf{1 4}$ by Concentration

sigmoid at $5 \mu \mathrm{M}$ is collected, however the other concentrations interrogated do not present data that can be fit to a $\mathrm{pK}_{\mathrm{a}}$. Exciting the minor absorbance band at $790 \mathrm{~nm}$ provides data that fits well to a sigmoid to give a $\mathrm{pk}_{\mathrm{a}} 5 \mu \mathrm{M}, 10 \mu \mathrm{M}$, and $20 \mu \mathrm{M}$, and provides the data presented in Table 2.3. Further, neither $\mathrm{pH}$ titrations at $2.5 \mu$, or interrogating the far red absorbance band at 890nm - at any concentration - provides data that can be fit to a $\mathrm{pK}_{\mathrm{a}}$.

Additionally, as opposed to 11 and $\mathbf{1 2}$, which present the same $\mathrm{pK}_{\mathrm{a}}$, and reliable data when they are titrated either from basic to acidic, or acidic to basic conditions, 14 does not present clear fit data when titration from basic conditions downward. 
All replicates had to be performed from acid conditions upward. In the context of the molecules explored in this work this was unusual.

\subsubsection{Unsuccessful Modifications}

With data describing both $\mathrm{pH}$ sensitivity, and concentration dependence in the fluorescence responses of $\mathbf{1 1}, \mathbf{1 2}$, and $\mathbf{1 4}$ and data that indicated a $\mathrm{pK}_{\mathrm{a}}$ could not be determined for $\mathbf{1 3}$, it seemed prudent to explore different methods of attaching either primary, or diamine groups to the cyanine scaffold. Diamines directly attached to the cyanine scaffold present a potentially interesting comparison in concentration dependence and $\mathrm{pH}$ dependence. Attaching the distal nitrogen through a ring, instead of an open chain, would provide a lower $\mathrm{pK}_{\mathrm{a}}$ in line with previously published work. However that work did not explore concentration dependence in the compounds synthesized therein, and titrations were performed in mixed aqueous/organic systems. The specific nature of the differences in concentration dependence between 11 and 12 solicits some interest, but the complete concentration dependent behavior of $\mathbf{1 4}$ was decidedly more interesting.

Distancing the $\mathrm{pH}$ sensitive diamine group from the cyanine core seemed to be a reasonable proposal, and the concentration dependence of $\mathbf{1 4}$ was not expected. To attempt to provide perspective on the data presented by $\mathbf{1 4}$ it was proposed to synthesize compounds which similarly placed a $\mathrm{pH}$ sensitive diamine group near, but not directly attached to, the conjugated $\pi$ system of the cyanine. Herein some of the difficulty of working with starting material $\mathbf{1 0}$ became more apparent. Being exceptionally robust chemically, $\mathbf{1 0}$ was also relatively resistant to modification at the meso-chloride position, which proved simple to circumvent through the addition 
of a transition metal catalyst. With the chloride being amenable to transition metal catalysis, it was proposed that any reasonable coupling partner could be utilized to modify the dye scaffold, with the caveat that the chemistry would restricted in solubility to aqueous, or highly polar solvents such as DMF or NMP. Further, as of this writing, this will be the first published report of modification of $\mathbf{1 0}$ in any manner, though reports of similarly tetra-sulfonated cyanines are present in the literature.

With the behavior of the phenyl-linked compound established, the obvious choices for potential analogs that would provide perspective on 14's behavior were compounds utilizing an array of chemically and spatially disparate linkers. Specifically, attaching a diamine group through either an alkyl, alkene, and alkyne functional group was first explored. The Negishi, Heck, and Sonogashira chemistry - respectively - that describes coupling of those various functional groups is well established, especially for the relatively simple coupling partners. Ideally 15, 16, and $\mathbf{1 7}$ would be the preferred compounds to explore the relationship between linker-type and behavior.

Of the three conceptually ideal compounds only $\mathbf{1 7}$ had a clearly available singlestep route to synthesis from commercially available starting materials. This was important functionally as all of the successfully synthesized derivatives of $\mathbf{1 0}$ showed tremendous insolubility in organic solvents, limiting the array of chemistry available to perform modification post coupling. Attempts to synthesize $\mathbf{1 6}$ was never undertaken directly, but attempts at compounds containing an alkene functional groups were made.

18 and 19 were the simplest and most coupling-friendly analogs of 15 and 16 that could be envisioned, being hypothetically accessible from palladium catalyzed coupling of phenyl acetylene or vinyl benzene to 10, respectively. Access to 18 proved 


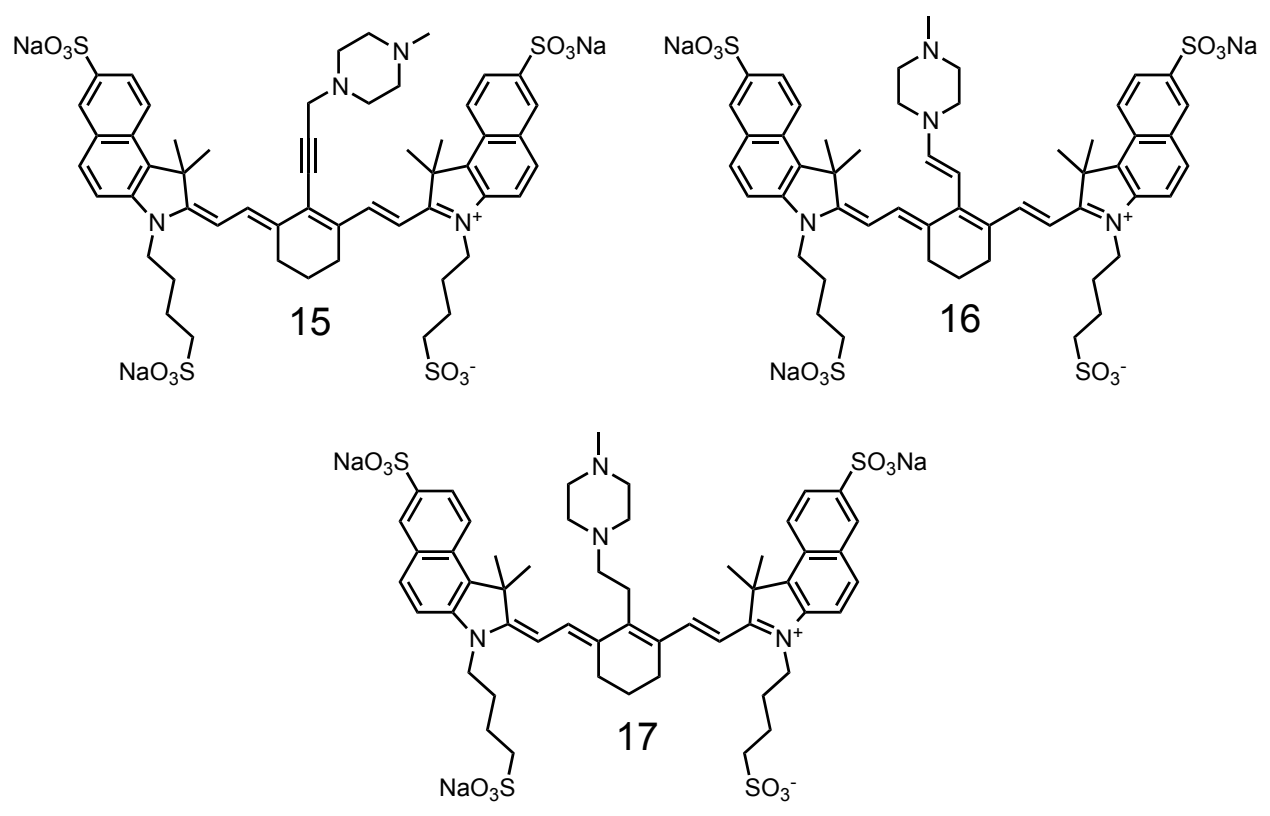

Figure 2.13: Proposed Methypiperazine-Cyanines

relatively simple under routine Sonogashira coupling conditions, however attempts at 19 provided little evidence of coupling, yielding only starting material. Though 18 was accessible, further Sonogashira coupling products proved elusive, Figure 2.15.

Attempts to synthesize $\mathbf{1 7}$ under both aqueous Negishi coupling conditions, and more standard conditions in DMF also proved unsuccessful. In an attempt to achieve a similarly $\mathrm{sp}^{3}$ carbon linked amine attached to the cyanine scafford, 22 was pursued from the alkyl boronic ester starting material, 2.16. Attempts at synthesizing 22 proved particularly frustrating, as the boronic ester coupling partner proved immiscible in aqueous conditions that provided $\mathbf{1 4}$. When the reaction was attempted in mixed $\mathrm{H}_{2} \mathrm{O} /$ DMF conditions the result was apparent rapid degradation of the starting material. This was consistent with attempts at optimizing the yield of $\mathbf{1 4}$ with use of DMF as a co-solvent. DMF is traditionally more friendly to coupling conditions that may be $\mathrm{O}_{2}$ sensitive, so effect of reducing reaction efficiency was unanticipated. 


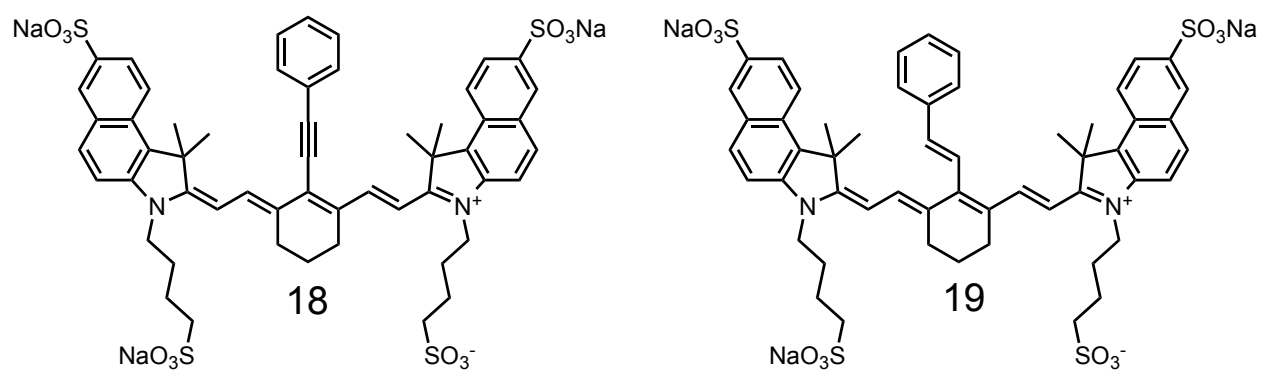

Figure 2.14: Proposed Test Modifications of 10

It could be hypothesized that converting the pinacol ester to the free boronic acid would provide a more water soluble coupling partner, but that conversion was never attempted due to the harsh conditions required to remove pinacol esters.

\section{$2.2 \quad$ Future Work}

Part of the problem with working with $\mathbf{1 0}$ is a lack of specific literature utilizing it as a starting material. There is a wealth of literature surrounding chemistry on cyanine type dyes of all flavors, but as of this writing, there are no published reports describing

chemistry on $\mathbf{1 0}$. The requirement of a transition metal catalyst to modify $\mathbf{1 0}$ at all was surprising, however it is a possibility that the resistance of the chloride position to addition-elimination chemistry that causes that necessity is also a contributing factor in $\mathbf{1 0}$ and it's derivatives being apparently more bench stable than cyanine derivatives handled in this lab previously.

The pursuit of an $\mathrm{sp}^{3}$ carbon as the initial atom appended to the $\pi$ system seems worthwhile, as it potentially allows for the design of complex binding groups which can be assembled initially, and then placed onto the cyanine scaffold. This relies on as yet unsuccessful attempts at coupling an alkyl-boron species to 10, Figure 2.16. 

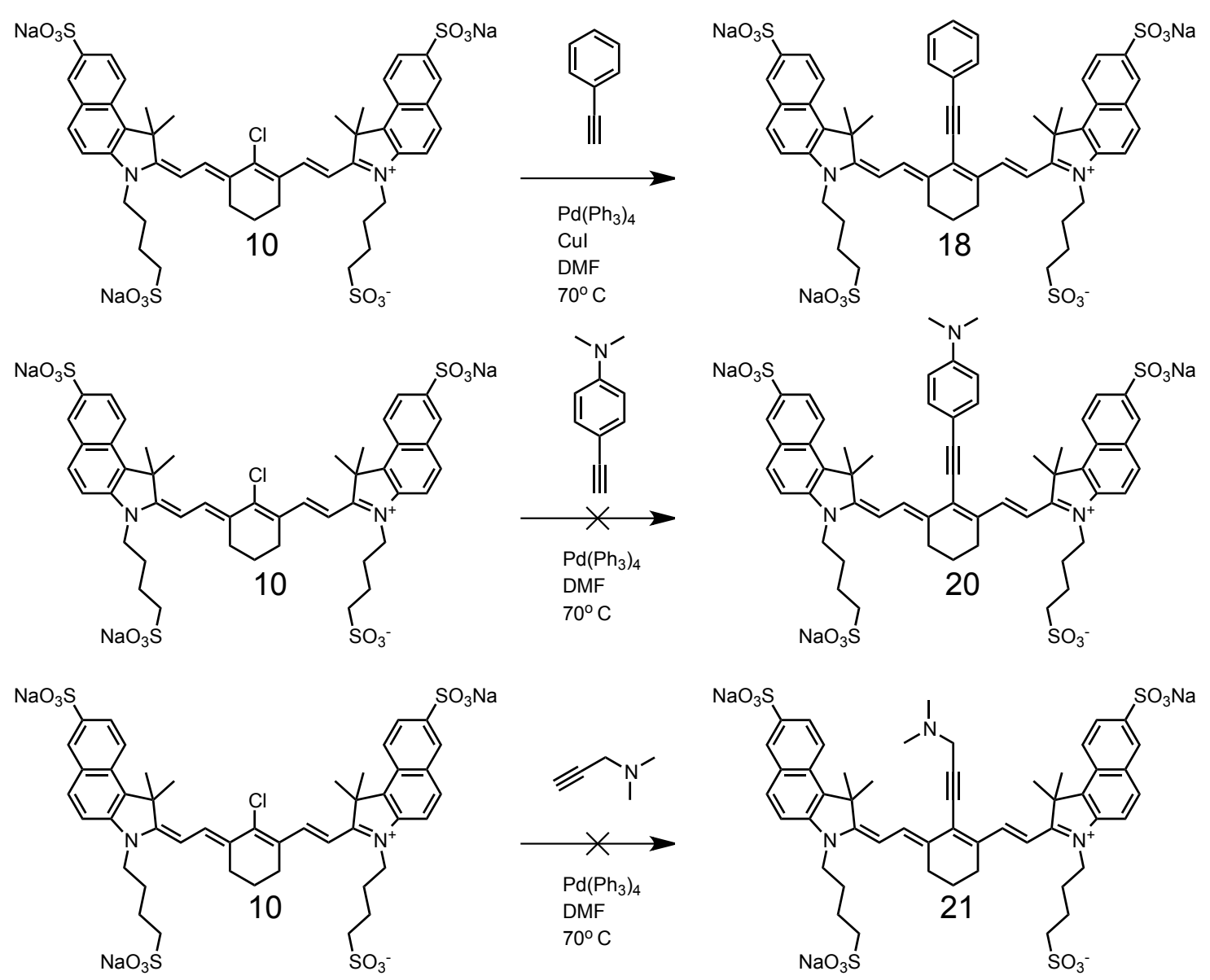

Figure 2.15: Sonogashira Chemistry on $\mathbf{1 0}$

Pursuing the successful coupling of relatively simple alkyl- and phenyl-boronic acids to increase the scope and understanding of potential coupling conditions is the most efficient approach at developing a knowledge base that would benefit future work utilizing $\mathbf{1 0}$ as a scaffold, Figure 2.17.

Another avenue of interest is 10's apparent instability to coupling with primary, as opposed to secondary amines, and an apparent instability to some diamine and triamine substrates. Attempts at coupling these amines to the cyanine scaffold either led to direct decomposition of the starting material, or isolation of a compound that 

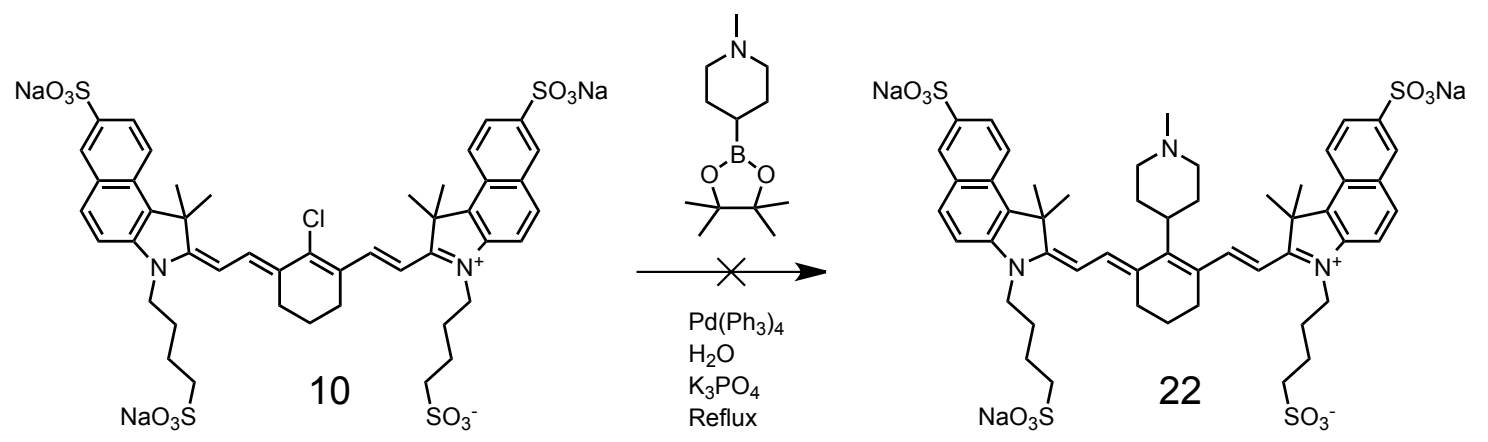

Figure 2.16: Attempts at Alkylboronic Ester Coupling to 10.

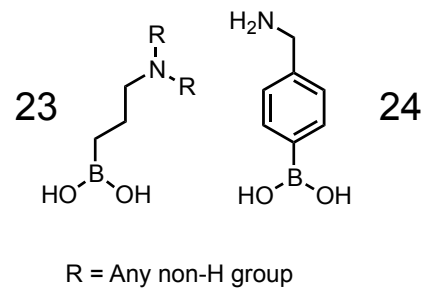

Figure 2.17: Examples of Boronic Acids whose coupling to 10 might be advantageous to establish.

exhibited absorbance profiles, and retention time under HPLC isolation conditions, that indicated successful coupling, but was not bench stable enough to characterize, Figure 2.18.

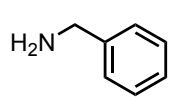

25

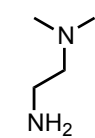

26<smiles>NCc1ccccn1</smiles>

27

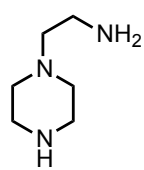

28

Figure 2.18: Examples of amines that decomposed 10 upon attempted coupling.

On the other hand, some amines would not couple to $\mathbf{1 0}$, despite attempts across a variety of coupling conditions. Pushing the coupling conditions to extremes in temperature, and varying ligand and catalyst loadings all returned starting matieral for these compounds, Figure 2.19. 


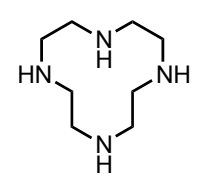

29

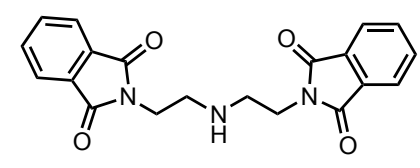

30

Figure 2.19: Examples of amines that returned solely starting material 10 upon attempted coupling.

Understanding the mechanisms of what leads some of these compounds to decompose $\mathbf{1 0}$ and that prevent others from coupling successfully would provide insight into how to expand the library of potential $\mathrm{pH}$ sensors based on this scaffold.

\subsection{1 $\mathrm{pH}$ Sensing as a Route Sensing Sugars}

Though there are many merits to sensing intracellular $\mathrm{pH}$, part of the goal of this work was to establish synthetic precedent for building complex sugar sensors out of a water soluble, near-IR scaffold. A pH sensitive cyanine serves as a reasonable starting point for this type of work because both the mechanism of fluorescence modulation for $\mathrm{pH}$ sensitivity, and the mechanism of fluorescence modulation for many sugar receptors is based on a PET mechanism, Figure 2.20.

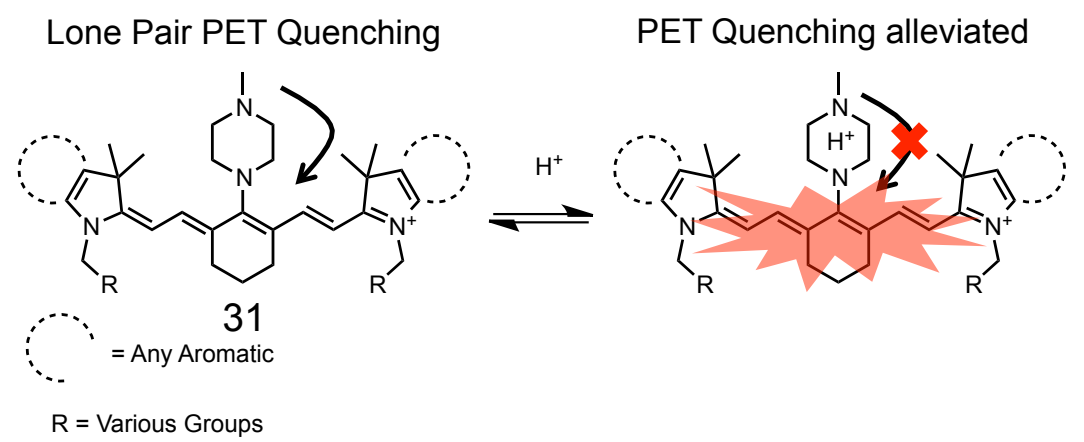

Figure 2.20: PET quenching in $\mathrm{pH}$ sensitive cyanines. 
Many fluorescent sugar sensors use boronic acid groups as a binding motif. Other examples exist, but boronic acids have become a favored route to sugar recognition. In $\mathrm{pH}$ sensitive fluorophores which rely on protonation of an amine as the mode of $\mathrm{pH}$ sensing, the availability of the amine lone pair to quench a fluorophore is directly related to concentration of protons in the environment that the fluorophore is surrounded by. As this concentration changes, the fluorescence is predictably modulated as the equilibrium between the amine and ammonium is shifted.

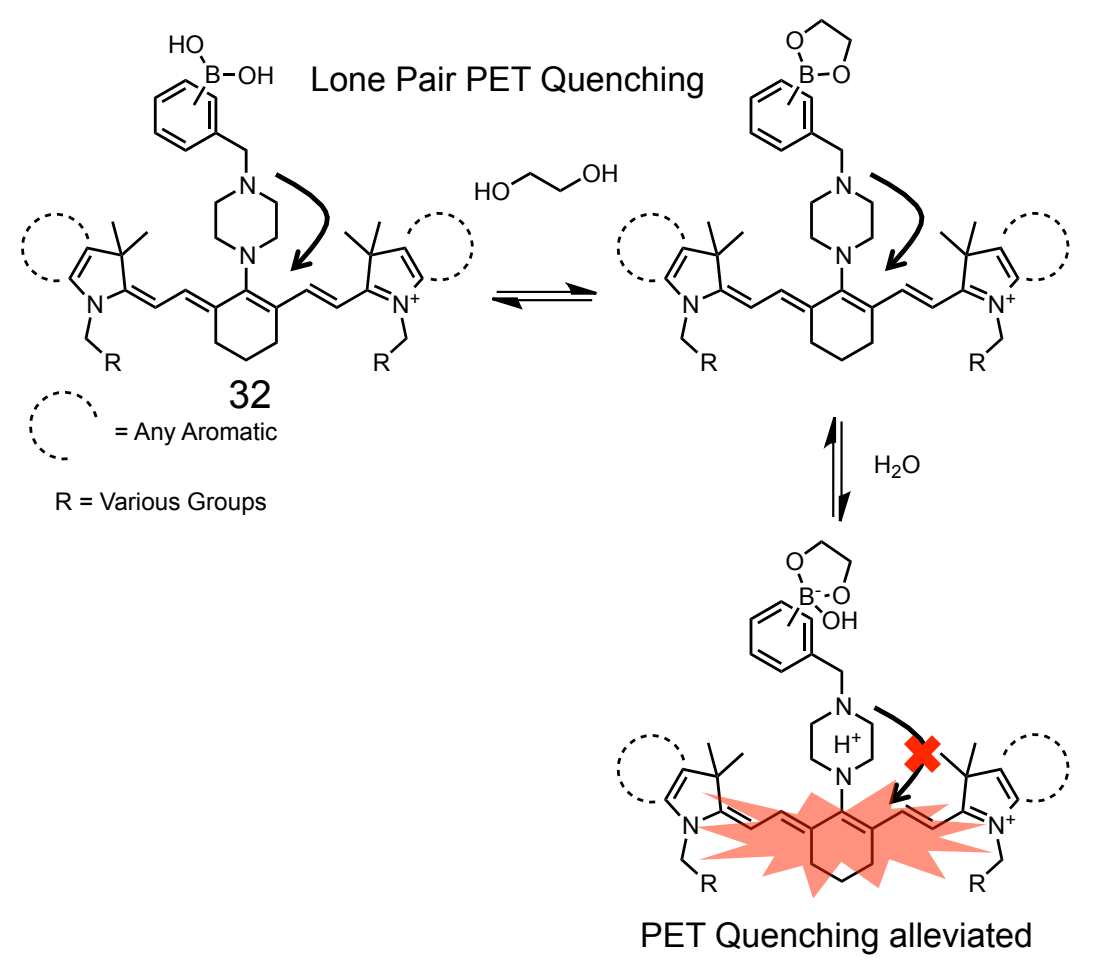

Figure 2.21: Alleviation of PET quenching from phenylboronic acid - diol interactions.

Phenylboronic acid based sugar sensors work under a similar mechanism. Attachment of a benzyl amine functional group provides a readily available lone pair to pet quench an attached fluorophore. Alcohols can replace the hydroxyl groups on boronic acids to form boronic esters relatively easily. Boronic acids and esters also readily 
convert to boronates. As sugars are functionally a series of polyols, boronic acids can bind to a single sugar or monosaccharide through hydroxyl displacement. Formation of the boronic ester increases the favorability of forming the boronate ester, which leads to protonation of a pendant amine. If the benzyl amine is located close enough to the boron, formation of a boronate will modulate the local $\mathrm{pH}$ enough to increase the favorability of protonating the amine, which then alleviates PET quenching, Figure 2.21 . 


\section{Chapter 3}

\section{Near IR Sugar Sensing}

\subsection{Fluorescent Chemical Detection of Sugars}

Detection of sugars in vivo is largely devoted to the detection of blood glucose levels in patients living with diabetes. Optical detection with a NIR sensor would provide an effective method of non-invasive glucose detection. To put this type of goal into perspective it is necessary to briefly examine why this hasn't yet been achieved, and what has been achieved in terms of monitoring blood glucose levels, which is significant.

The current method for self-monitoring blood glucose levels relies on obtaining a capillary blood sample at regular intervals. This sample is applied to a test strip which is impregnated with glucose oxidase, and a peroxide sensitive chromophore. The oxidation of glucose releases peroxide, which induces a color change in the test strip. The color change can then be monitored by a simple hand held detector. 
Though accurate, this method presents some problems. As it is an active sampling method, it cannot be performed while asleep, or performing complicated tasks such as driving a car. Additionally, as the test itself is invasive, requiring multiple samplings per day, the discomfort of the actual act of obtaining blood impinges upon patient sampling fidelity.

Development of a minimally invasive method of monitoring blood glucose levels would alleviate the aforementioned concerns. Improving fidelity of monitoring of blood glucose levels would decrease instances of hypoglycemia and hyperglycemia, which lead to the chronic complications and morbidities associated with diabetes.

This has been approached in a multitude of ways. Implanted amperometric sensors have been explored, but require frequent re-calibration against finger-prick test blood glucose levels $[44,45]$. Reverse Iontophoresis has also been explored, but similary suffers from accuracy issues, and inflicts significant skin irritation [46, 47]. Both of these methods have produced commercially available devices, but the drawbacks associated with them have hindered widespread acceptance. Also, electrochemical sensors for glucose embedded in contact lenses have been explored recently. Being based on the same enzymatic methods as glucose test strips, they are similarly accurate, unfortunately glucose concentrations in tear films are significantly lower than serum glucose levels, $0.1-0.6 \mu \mathrm{M}$ as opposed to 4-6 $\mu \mathrm{M}[48,49]$. Implantable nanosensors based on hydrogels, and "smart-tattoo" biosensors have also been explored, but are notably in their infancy $[50,51,52]$.

Electrochemical and enzymatic detection of glucose are popular in part because of the accuracy associated with sensing mechanisms, and the simplicity associated with detection events. The aforementioned methods all have the advantage of being easily 
performed by anyone, regardless of technical training. However they present little in the way of decreased invasiveness as a method of monitoring. Fluorescent sensors, particularly NIR sensors afford a clear path to minimally, or non-invasive detection of glucose. NIR fluorescence would be ideal because it would place excitation, and emission wavelengths above the absorption of hemoglobin. Detection can be achieved optically, as long wavelength light has sufficient tissue penetration to excite a fluorophore deeply embedded in vivo. To this end, fluorescent detection of sugars in general, and glucose in particular has been pursued $[53,54]$.

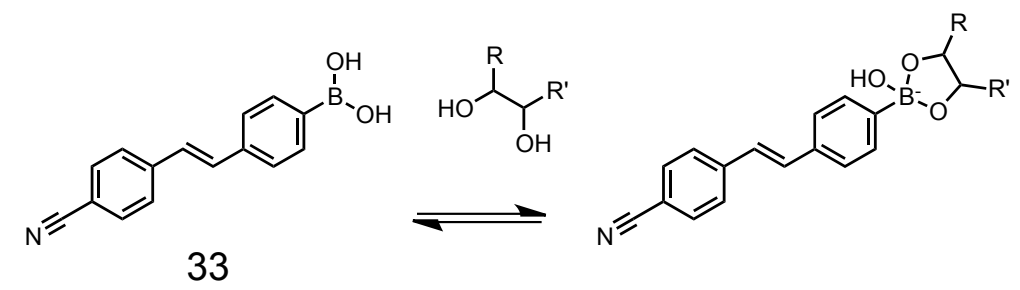

Figure 3.1: 4'-cyanostilbene-4-boronic acid, (CSTBA)

Fluorescent detection of glucose is largely pursued through phenylboronic acids, as they allow for reversible binding of diols in general, and sugars in particular [55, 56]. This interaction can be exploited to provide either PET-based, or Internal Charge Transfer (ICT)-based sensors for sugars. ICT-based fluorescent detection of glucose can be illustrated by a pair of examples of stilbene derivatives published by Lakowicz and coworkers. 33, an electron poor stilbene derivative named CSTBA after its structure provides a significant redshift in fluorescent emission upon forming a boronate ester when titrated with sugars. Emission shifts from $388 \mathrm{~nm}$ to $455 \mathrm{~nm}$, when excited at 325nm, Figure 3.1 [57]. Meanwhile, an electron-rich stilbene behaves differently. When appended with a dimethylamino group, upon binding a sugar, fluorescent emission blue shifts from $515 \mathrm{~nm}$ to $450 \mathrm{~nm}$, when excited at 330nm, Figure 3.2 [58]. 


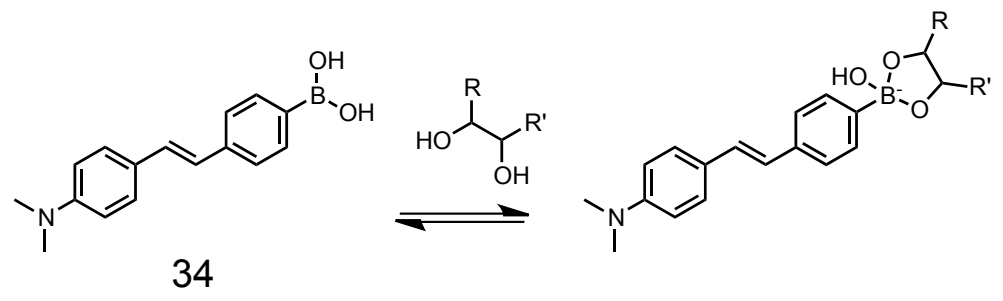

Figure 3.2: 4'-(dimethylamino)stilbene-4- boronic acid, (DSTBA)

For 34, DSTBA, the blue shift in fluorescence emission is accompanied by a slight increase in fluorescence intensity. This is attributed by Lakowicz and coworkers to a decrease in the boronic acid's ability to serve as an electron withdrawing group when the boronate is formed.

Fluorescence based sugar detection can also be performed via a PET mechanism, as opposed to an ICT mechanism. Shinkai and coworkers published $\mathbf{3 5}$ describing a scheme where formation of a boronate-sugar complex decreases the local $\mathrm{pH}$ to favor protonation of an amine. This protonation leads to alleviation of PET quenching of an anthracene fluorophore, Figure 3.3 [59].
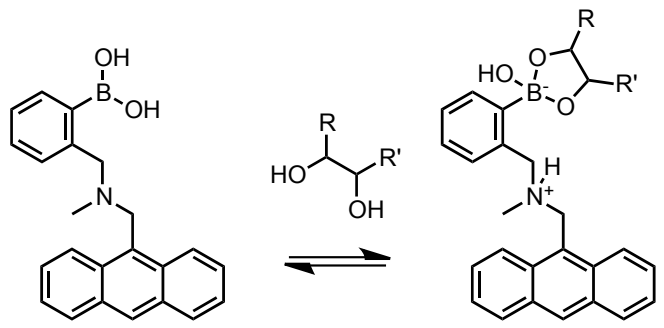

35

Figure 3.3: Shinkai's Anthracene Based PET System

Shinkai's sensor 35 provided a $\mathrm{K}_{\mathrm{d}}$ of $15 \mathrm{mM}$ for glucose in $33 \% \mathrm{MeOH}$. 35, like most mono-boronic acid based receptors however showed stronger selectivity for fructose than for glucose. Shinkai and coworkers therefore pursued a bis-boronic acid 
based receptor based on the same platform. 36 was obtained relatively simply and showed both greater selectivity for glucose than $\mathbf{3 5}$ and a stronger binding constant for glucose under similar titration conditions, Figure 3.4 [60].
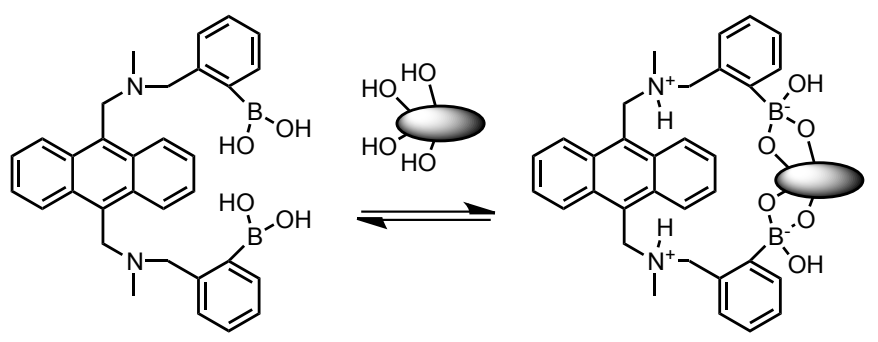

36

Figure 3.4: Shinkai's Anthracene Based Bisboronic Acid Receptor

Designing a sensor which selectively binds glucose is a slightly more difficult task than designing a sensor which just binds glucose well. It turns out that phenylboronic acid receptors with a single boronic acid group have a stronger affinity for both fructose and galactose than glucose. Skinkai's sensor received fairly quick competition from James and coworkers who designed a series of fluorescent sensors using a pair of phenylboronic acid groups linked by alkane chains, Figure $3.5[61,62]$.
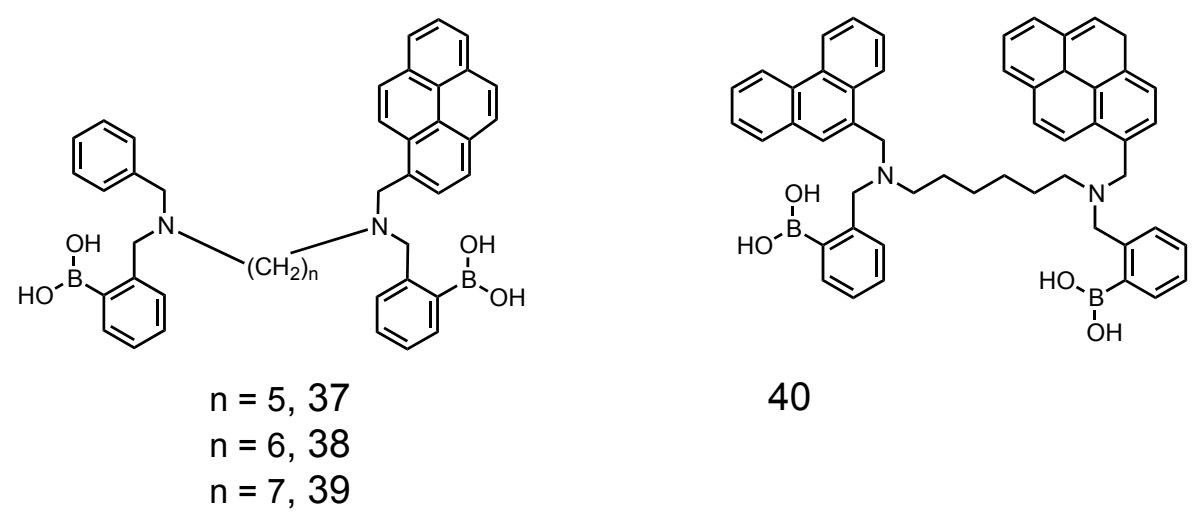

40

Figure 3.5: Intramolecular Energy Transfer Based Glucose Sensors 
James' sensors provide a fluorescence increase upon binding simple monosaccharide sugars, and provides some qualitative information about designing glucose $s e$ lective receptors. Varying the lengths of the alkane spacer in $\mathbf{3 7}, \mathbf{3 8}$, and 39 give a varying degree of selectivity for glucose over fructose and galactose, as well as the varying direct sensitivity for glucose. 38 with a six carbon linker between the nitrogens provides the strongest selectivity for glucose of this group of compounds. 40 was eventually synthesized based on the flexible six carbon linker, pairing a phenanthrene with the pyrene, increasing fluorescence intensity and emission wavelengths.

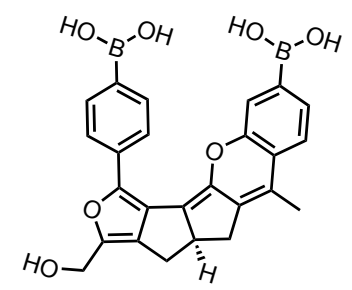

41

Figure 3.6: Intramolecular Energy Transfer Based Glucose Sensors

Another approach towards designing a glucose selective fluorescent phenylboronic acid based sensor, highlighted by Drueckhammer and coworkers, was to utilize computer modeling to predict the most effective binding motif possible [63]. Using a model based on the optimal bonding geomtry of two generic phenylboronic acids to glucopyranose, Sensor 41 was conceived and synthesized in seven steps to produce what is currently the most selective chemical sensor for glucose, Figure 3.6.

Drueckhammer's sensor is doubly interesting though, as binding events favor the pyranose form of glucose. Previous work by Norrild and coworkers determined that, at least with $\mathbf{3 6}$ bisboronic acids bind glucose initially as the pyranose, but isomerization 
occurs leading to the stably bound sugar being in the furanose form[64].

\begin{tabular}{|c|c|c|c|c|c|c|c|c|c|}
\hline & 33 & 34 & 35 & 36 & 37 & 38 & 39 & 40 & 41 \\
\hline $\mathrm{K}_{\mathrm{d}} \mathrm{Glu}$ & $\begin{array}{l}98.0 \\
\mathrm{mM}\end{array}$ & $\begin{array}{l}43.0 \\
\mathrm{mM}\end{array}$ & $\begin{array}{l}15.8 \\
\mathrm{mM}\end{array}$ & $\begin{array}{c}251.1 \\
\mu \mathrm{M}\end{array}$ & $3.0 \mathrm{mM}$ & $1.0 \mathrm{mM}$ & $2.9 \mathrm{mM}$ & $9.6 \mathrm{mM}$ & $25 \mu \mathrm{M}$ \\
\hline $\begin{array}{l}\mathrm{K}_{\mathrm{d}} \\
\text { Fru }\end{array}$ & $2.5 \mathrm{mM}$ & $1.0 \mathrm{mM}$ & $1.0 \mathrm{mM}$ & $3.1 \mathrm{mM}$ & $2.3 \mathrm{mM}$ & $1.2 \mathrm{mM}$ & $1.3 \mathrm{mM}$ & $8.0 \mathrm{mM}$ & - \\
\hline $\begin{array}{c}\text { Titration } \\
\text { Solvent }\end{array}$ & $\begin{array}{c}33.3 \% \\
\mathrm{MeOH} \\
@ \mathrm{pH}= \\
8.0\end{array}$ & $\begin{array}{c}33.3 \% \\
\mathrm{MeOH} \\
@ \mathrm{pH}= \\
8.0\end{array}$ & $\begin{array}{c}33.3 \% \\
\mathrm{MeOH} \\
@ \mathrm{pH}= \\
7.7\end{array}$ & $\begin{array}{c}33.3 \% \\
\mathrm{MeOH} \\
@ \mathrm{pH}= \\
7.7\end{array}$ & $\begin{array}{c}52 \% \\
\mathrm{MeOH} \\
@ \mathrm{pH}= \\
8.2\end{array}$ & $\begin{array}{c}52 \% \\
\mathrm{MeOH} \\
@ \mathrm{pH}= \\
8.2\end{array}$ & $\begin{array}{c}52 \% \\
\mathrm{MeOH} \\
@ \mathrm{pH}= \\
8.2\end{array}$ & $\begin{array}{c}52 \% \\
\mathrm{MeOH} \\
@ \mathrm{pH}= \\
8.2\end{array}$ & $\begin{array}{l}30 \% \\
\mathrm{MeOH} \\
@ \mathrm{pH} \\
=7.5\end{array}$ \\
\hline $\operatorname{Ex} \lambda:$ & $330 \mathrm{~nm}$ & $335 \mathrm{~nm}$ & $370 \mathrm{~nm}$ & $370 \mathrm{~nm}$ & $342 \mathrm{~nm}$ & $342 \mathrm{~nm}$ & $342 \mathrm{~nm}$ & $342 \mathrm{~nm}$ & $375 \mathrm{~nm}$ \\
\hline $\operatorname{Em} \lambda:$ & $\begin{array}{c}450 \mathrm{~nm} / \\
515 \mathrm{~nm}\end{array}$ & $\begin{array}{c}388 \mathrm{~nm} / \\
455 \mathrm{~nm}\end{array}$ & $423 \mathrm{~nm}$ & $423 \mathrm{~nm}$ & $397 \mathrm{~nm}$ & $397 \mathrm{~nm}$ & $397 \mathrm{~nm}$ & $417 \mathrm{~nm}$ & $447 \mathrm{~nm}$ \\
\hline
\end{tabular}

Table 3.1: Experimentally determined $\mathrm{K}_{\mathrm{d}} \mathrm{s}$ and conditions for cited sugar sensors

As a relatively brief overview of fluorescent chemical sensing for glucose, several things become apparent from the work cited here. Glucose is difficult to sense chemically, with fructose, galactose, and ascorbic acid binding to phenylboronic acids being more favored. Though only Drueckhammer's sensor 41 requires an even remotely lengthy synthesis, all of the sensors cited append the phenylboronic acid moiety at the end of their synthesis, indicating that performing further chemistry after that step, or appending the binding motif early in a synthesis is inadvisable. The cited fluorescent sensors, with the exception of CSTBA 33 present fluorescence intensity increases upon titration with sugars, however all titrations were performed in fairly high concentrations of organic co-solvents. Additionally many of the titrations with sugars were performed at non-physiologically relevant pHs, Table 3.1. 


\subsection{NIR Sugar Sensing}

The current literature on glucose detection specifically, or sugars in general, is devoid of examples of chemical NIR fluorescent sensors. However, several nanosensor approaches to NIR glucose detection have been undertaken, including using modified Single Walled Carbon Nanotubes (SWCNs), fluorescent microgels, and quantum dots $[65,66,67]$.

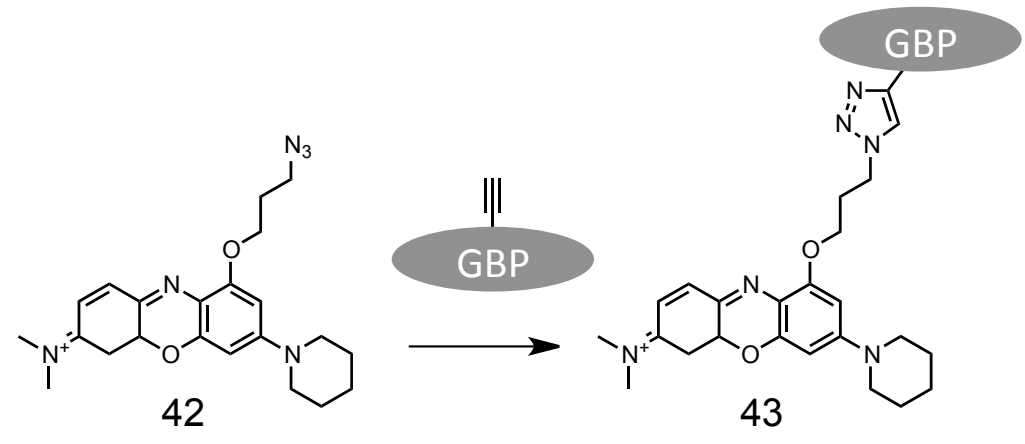

Figure 3.7: NIR Glucose Sensitive Bioconjugate

Though these sets of examples rely on some of the same principles as the chemical sensors described previously, the SWCNs and quantum dots, which are large macromolecular assemblies behave very differently in solution from small molecule fluorophores, limiting direct comparisons. At least one series of NIR bioconjugate sensors has been published utilizing CuAAC chemistry to append 651-Blue Oxazine 42 to various positions on glucose/galactose binding protein (GBP) to provide bioconjugates represented by $\mathbf{4 3}$, Figure 3.7 [68]. Changes in conformation of the protein as it binds glucose change the bulk solvent environment around the fluorophore - which is environment sensitive. As the environment changes, fluorescence intensity changes. 


\subsubsection{Designing a NIR Cyanine Based Sugar Sensor}
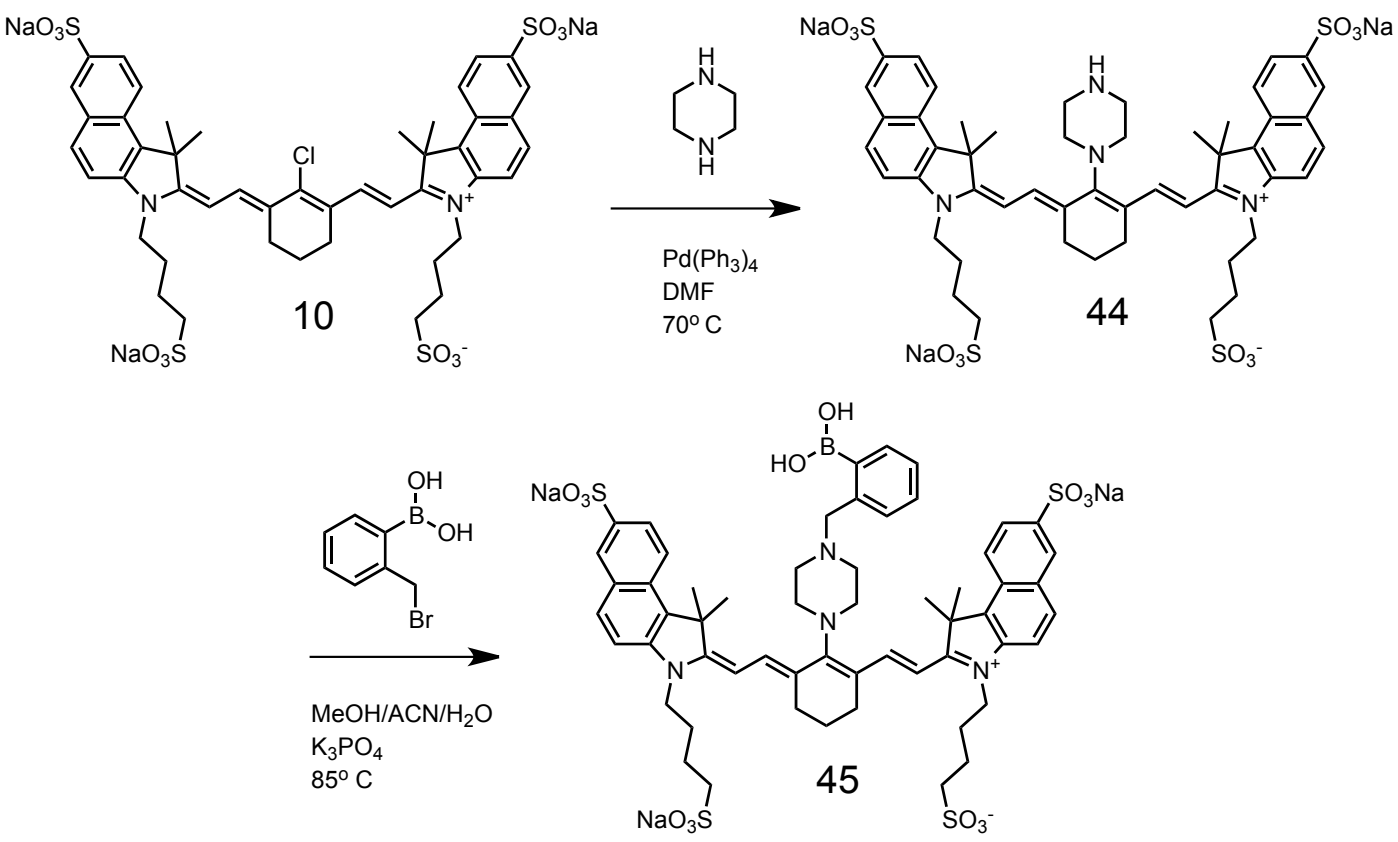

Figure 3.8: Cyanine Based Sugar Sensor

Sensor 45 was synthesized in two steps from 10, with 44 isolated crude and identified by H-NMR before being used as is. The specific reasons for picking $\mathbf{1 0}$ as the starting material, being tetra-sulfonated, limits the methods for purification to almost solely HPLC. At least one group has published procedures that indicate partially successful purification of a different tetra-sulfonated cyanine core with Solid Phase Extraction (SPE), but that is a single example, and HPLC is still used for final purification [69]. Also, even though 10 shows at least effective solubility in DMF, derivatives of $\mathbf{1 0}$ are extremely insoluble in organic solvent systems and mixed systems with high percentages of organic solvents.

Attempts at dealing with this solubility issue can been seen in the synthesis of $\mathbf{4 5}$, where a three solvent system of Acetonitrile, Methanol, and Water was used to both 
solubilize the starting material, and ensure an environment conducive to alkylation of a secondary amine. With these various hurdles in synthesis, $\mathbf{4 5}$ was the sole sugar binding compound that was achieved during the course of this work. In some sense, it was surprising that $\mathbf{4 5}$ was the only compound that could be made, considering that it was relatively easy to synthesize once the alkylation was accomplished. It was only two steps, both purified via HPLC, neither with exceptionally complex mechanistic chemistry, or harsh or complicated conditions.

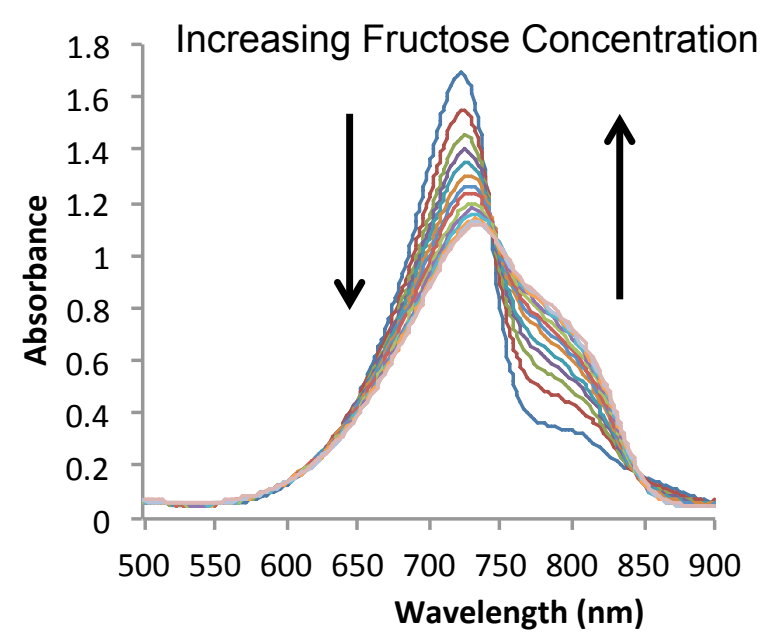

Figure 3.9: Absorbance Titration of $\mathbf{4 5}$ with Fructose

Absorbance titrations of $\mathbf{4 5}$ with fructose provide noticeable differences from it's closest $\mathrm{pH}$ sensor analog, 11, though a single absorbance peak dominates in the unbound state, which mirrors the deprotonated state of $\mathbf{1 1}$, upon binding and assumedly protonation of the piperazine amines, only a single absorbance band is observed gaining intensity. Also unlike $\mathbf{1 1}$ the dominant absorption band from the starting material does not completely disappear when $\mathbf{4 5}$ appears fully saturated by fluorescence.

When titrated with glucose and fructose $\mathbf{4 5}$ provides relatively poor binding bind- 

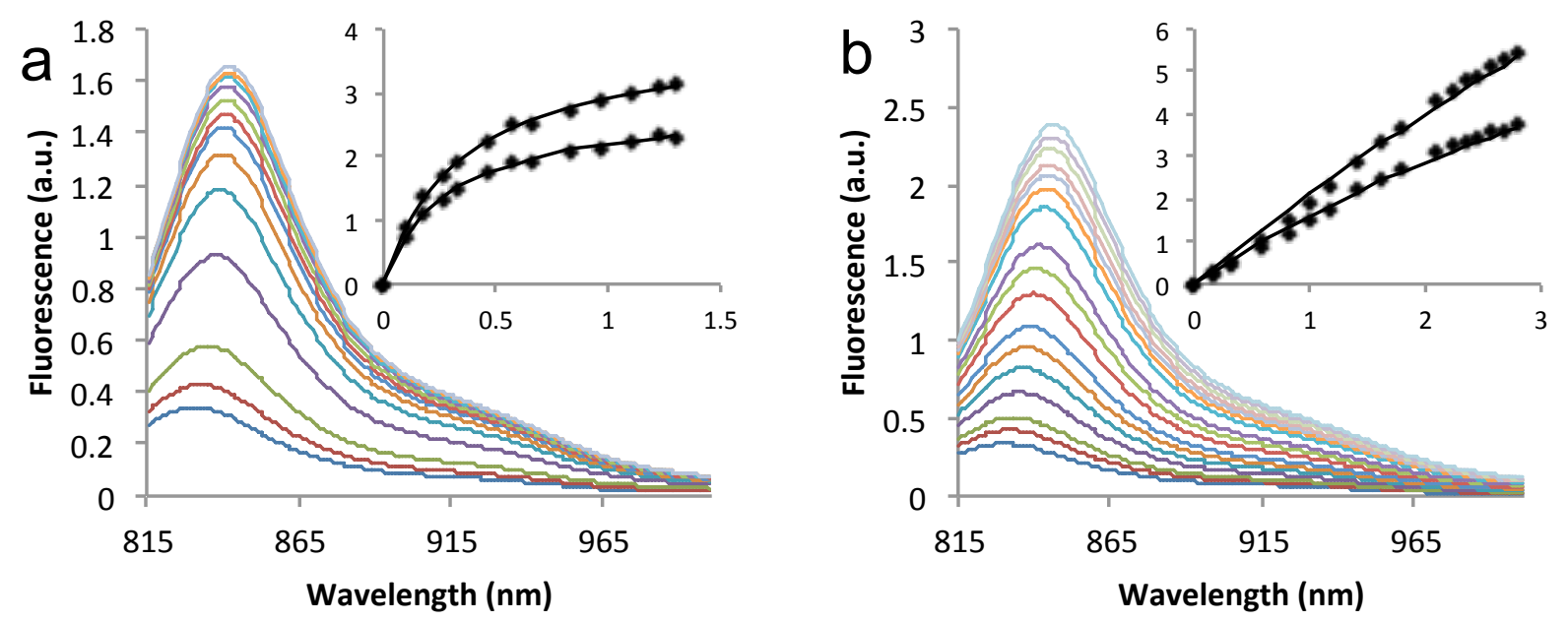

Figure 3.10: Fluorescence titrations of $\mathbf{4 5}$ with Glucose (b) and Fructose (a), in 165 $\mathrm{mM}$ PBS, excitation wavelength at $780 \mathrm{~nm}$. Insets are Fluorescence intensity $\left(\mathrm{I} / \mathrm{I}_{\mathrm{o}}\right)$ at $825 \mathrm{~nm}$ and $835 \mathrm{~nm}$ plotted against concentration of free analyte (M).

ing constants compared to previously published sugar sensors. As expected being a mono-boronic acid based sensor, 45 provided a stronger binding constant for fructose than for glucose, but the binding constant for fructose provided was more than an order of magnitude weaker than previously published sensors, Figures 3.9, 3.10. Binding of Glucose in particular is so weak that it is unclear whether the fluorescence changes observed are actual binding events, or solely a result of viscosity changes, Table 3.2, Figure 3.10. It would be logical to assume that the observed fluorescence changes are potentially a combination of both, but sufficient controls to determine how viscosity effects are affecting fluorescence were not performed.

Though the fluorescence data for fructose is strongly indicative of binding, an unusual quirk of the data acquired is how dependent the measured binding constant is upon where emission is measured. This suggests there are two events occuring within the titration system that are affecting the fluorescence output. The simplest 
scenario is one in which the apparent red shift in the fluorescence emission upon addition of glucose and fructose is dependent upon one event, and the changes in fluorescence intensity are dependent upon another.

Considering the exceptionally high concentration of fructose and glucose being used in the titrations, viscosity effects cannot be discounted as affecting the results presented.

\begin{tabular}{|c|c|c|c|c|}
\cline { 2 - 5 } \multicolumn{1}{c|}{} & \multicolumn{2}{c|}{ Glucose } & \multicolumn{2}{c|}{ Fructose } \\
\hline$E m \lambda:$ & $825 \mathrm{~nm}$ & $835 \mathrm{~nm}$ & $825 \mathrm{~nm}$ & $835 \mathrm{~nm}$ \\
\hline $\mathrm{K}_{\mathrm{d}}$ & $6.6 \mathrm{M}$ & $15.5 \mathrm{M}$ & $282.2 \mathrm{mM}$ & $343.2 \mathrm{mM}$ \\
\hline $\mathrm{B}_{\max }$ & $12.3 \mathrm{M}$ & $34.4 \mathrm{M}$ & $2.8 \mathrm{M}$ & $3.9 \mathrm{M}$ \\
\hline
\end{tabular}

Table 3.2: Binding and Saturation Constants for $\mathbf{4 5}$

The exceptionally poor binding constants can possibly be attributed to the $\mathrm{pK}_{\mathrm{a}}$ of the boronic acid, or the fact that sugar binding titrations were performed in entirely aqueous conditions. The effect of boronic acid $\mathrm{pK}_{\mathrm{a}}$ on boronate, and boronate ester formation has been explored extensively [70], and this seems a likely contributor to poor binding. However, few chemical sensors for sugars have been interrogated in fully aqueous systems, leaving this possibility unexplored.

\subsubsection{Attempts at Building Complex Sugar Binding Motifs}

Sensor 45 was the only sugar binder achieved in this work, but it was not the only one pursued. Sensor 46 was pursued in an effort to install a phenylboronic acid group with a lower $\mathrm{pK}_{\mathrm{a}}$, which would assumedly improve binding to sugars. Sensor $\mathbf{4 6}$ was achieved in much the same manner as $\mathbf{4 5}$, but initial titration experiments indicated 
even poorer binding results than it's predecessor, so it was not fully characterized.

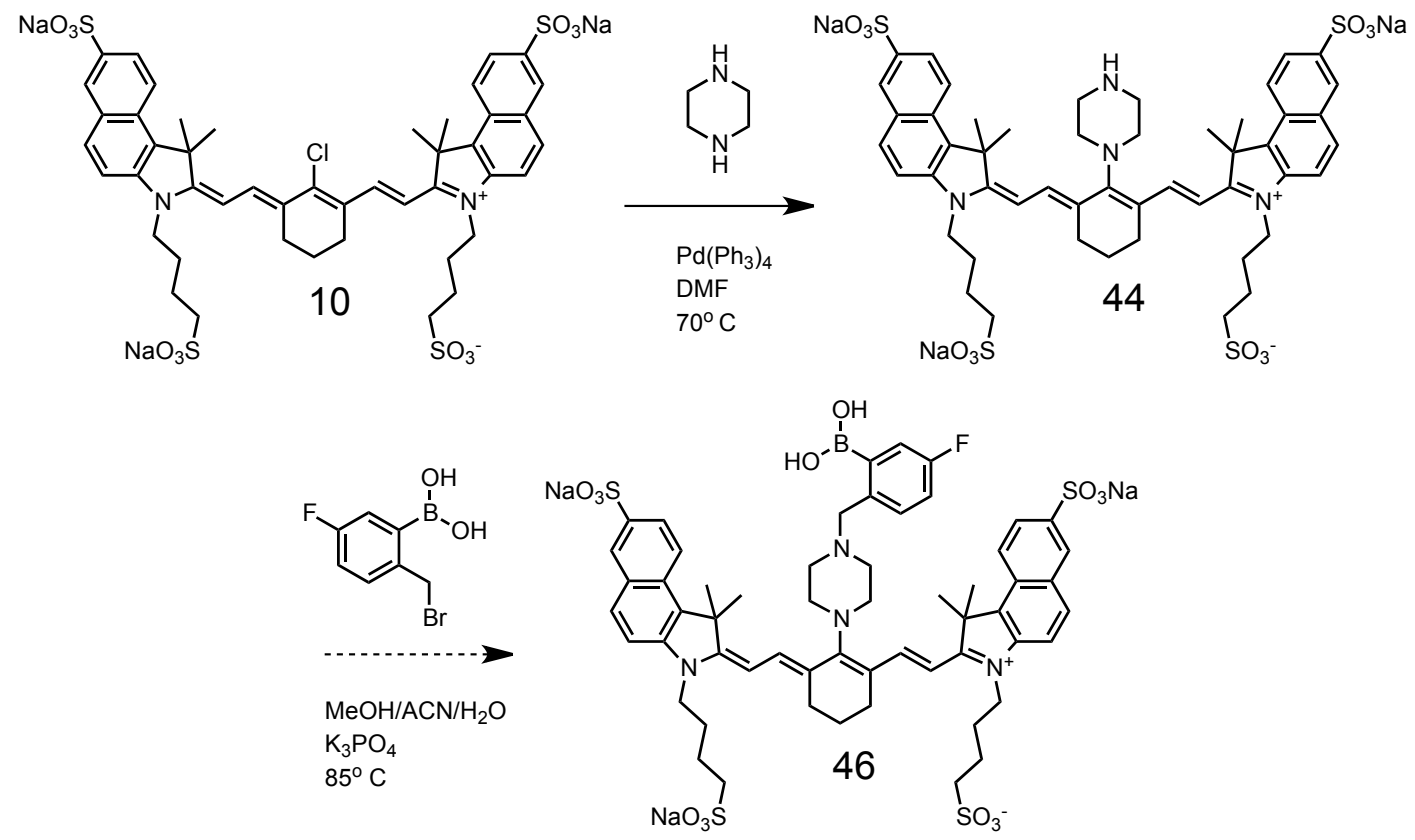

Figure 3.11: Cyanine Based Sugar Sensor

Attempting to build a simple bis-boronic acid sensor proved difficult. Proposed Sensor 48 was envisioned as a simple test case, being a close analog to $\mathbf{4 5}$. Intermediate 47 proved more difficult than expected to isolate, but was eventually isolated to provide relatively convincing spectroscopic characterization. Unfortunately, attempts at alkylating 47 only yielded decomposition.
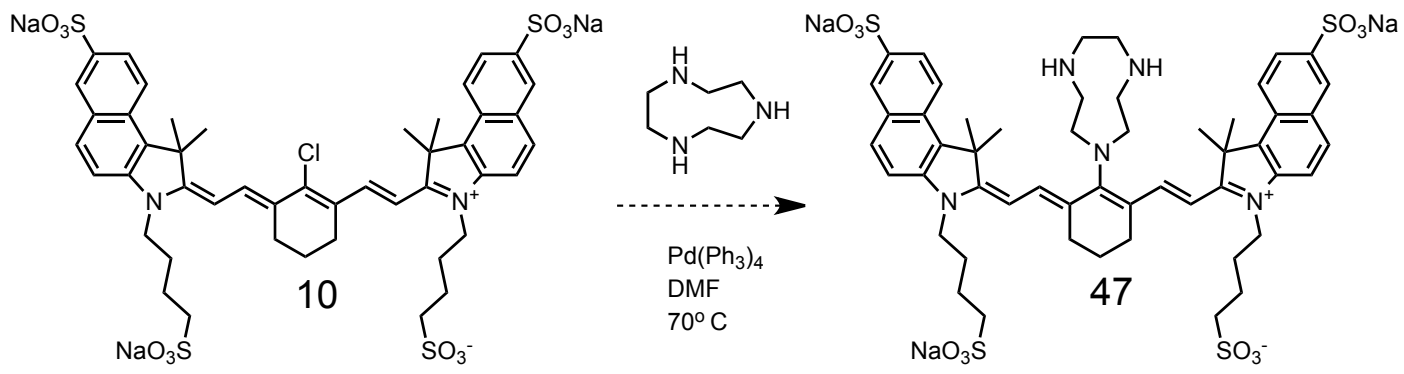

Figure 3.12: Bis-secondary amine intermediate 
Another bis-amine precursor was envisioned in 49, appending phthalimide protected amines which could be deprotected and either alkylated, or coupled to. However 49 could not be achieved despite attempting a wide range of conditions and surveying other palladium catalysts. It was hypothesized that the protected bisphthalimide may have been too bulky of a ligand for palladium catalysis at such a crowded center.

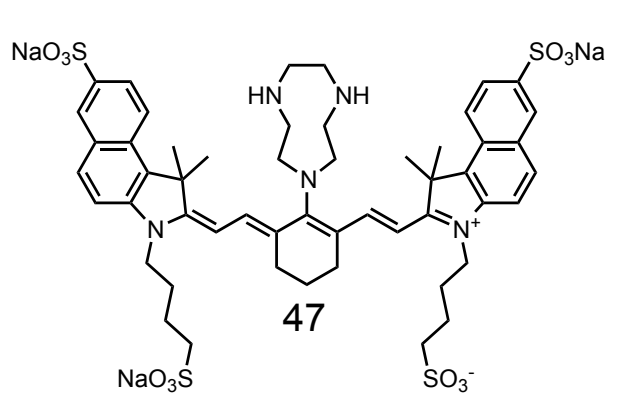

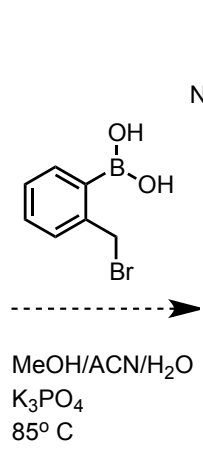
$85^{\circ} \mathrm{C}$

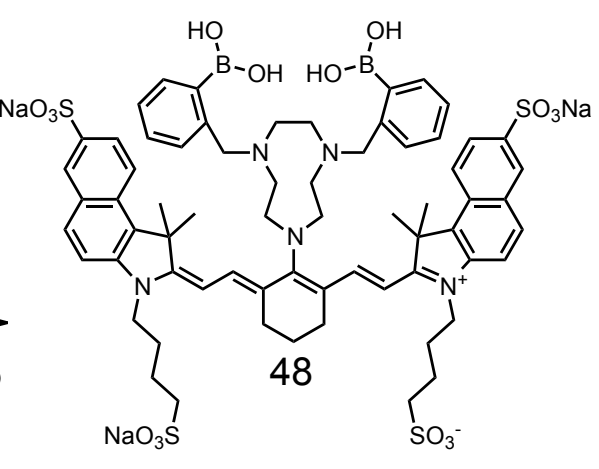

Figure 3.13: Unsuccessful Bisboronic Acid 48

50 was also envisioned, but only as a compound which could potentially put the concentration dependence of $\mathbf{1 5}$ into context. Initially, the reaction appeared successful, providing an absorbance spectra indicative of successful coupling, during reaction monitoring, and providing a single product by HPLC. However despite several attempts with indications of the desired product, H-NMR confirmation of the product could be achieved.

Similarly, $\mathbf{5 2}$ was attempted along the same lines as 45 and $\mathbf{4 6}$, unfortunately elutions obtained off the HPLC, though providing absorbance profiles indicative of the compound of interest, were acceptably pure by H-NMR. As with $\mathbf{5 0}$ single isolates off the HPLC were achieved with absorbance profiles indicative of a single cyanine derivative, but significant aromatic impurities could not be effectively removed 


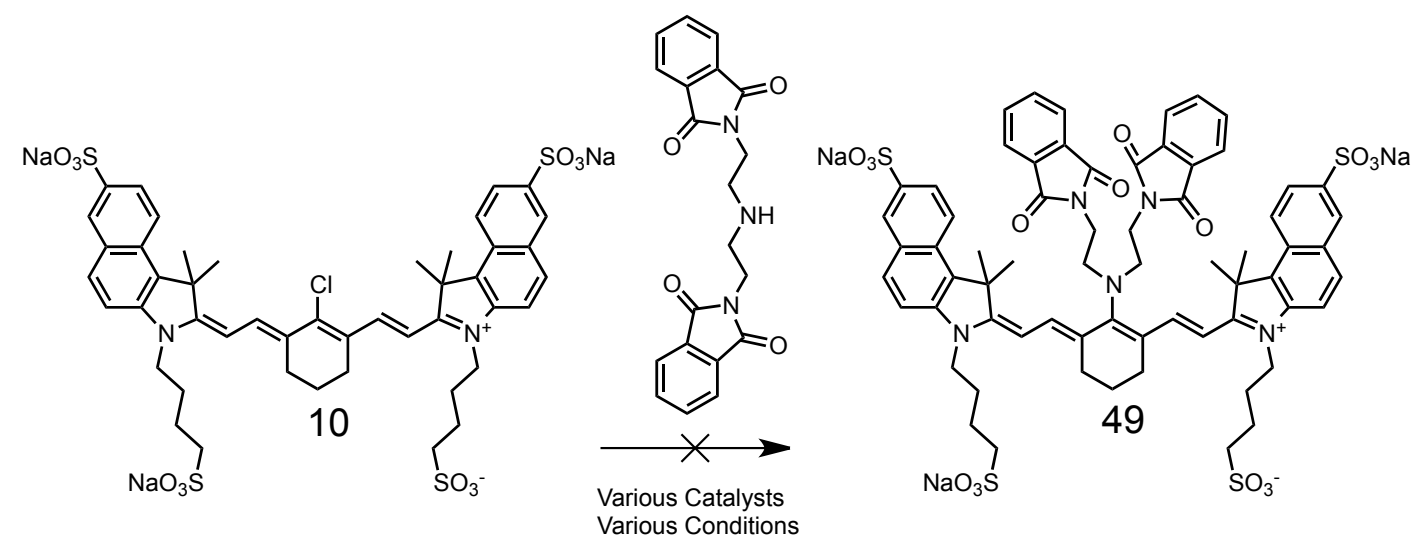

Figure 3.14: Unsuccessful Bis-secondary Amine Intermediate 49

through chromatography.
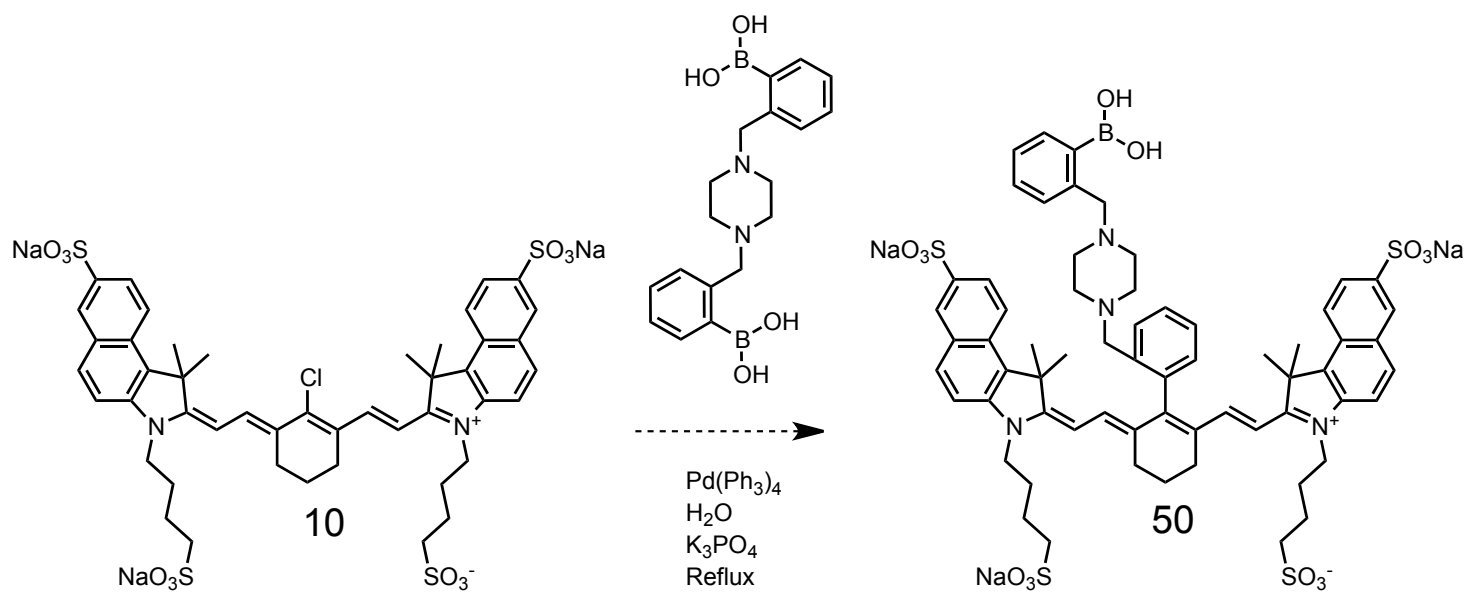

Figure 3.15: Unsuccessful Phenylboronic Acid Compound 50

The array of chemistry presented here highlights a few of the difficulties of working with 10 as a starting material. Some reactions work exceptionally well, and in the case of 11 and other compounds achieved with very simple secondary amines, the chemistry appears robust to adventitious oxygen or water during the reaction.

Similarly, $\mathbf{1 5}$ is achieved through Suzuki chemistry in a fully aqueous system, although performing this reaction under air free conditions was never attempted, it 

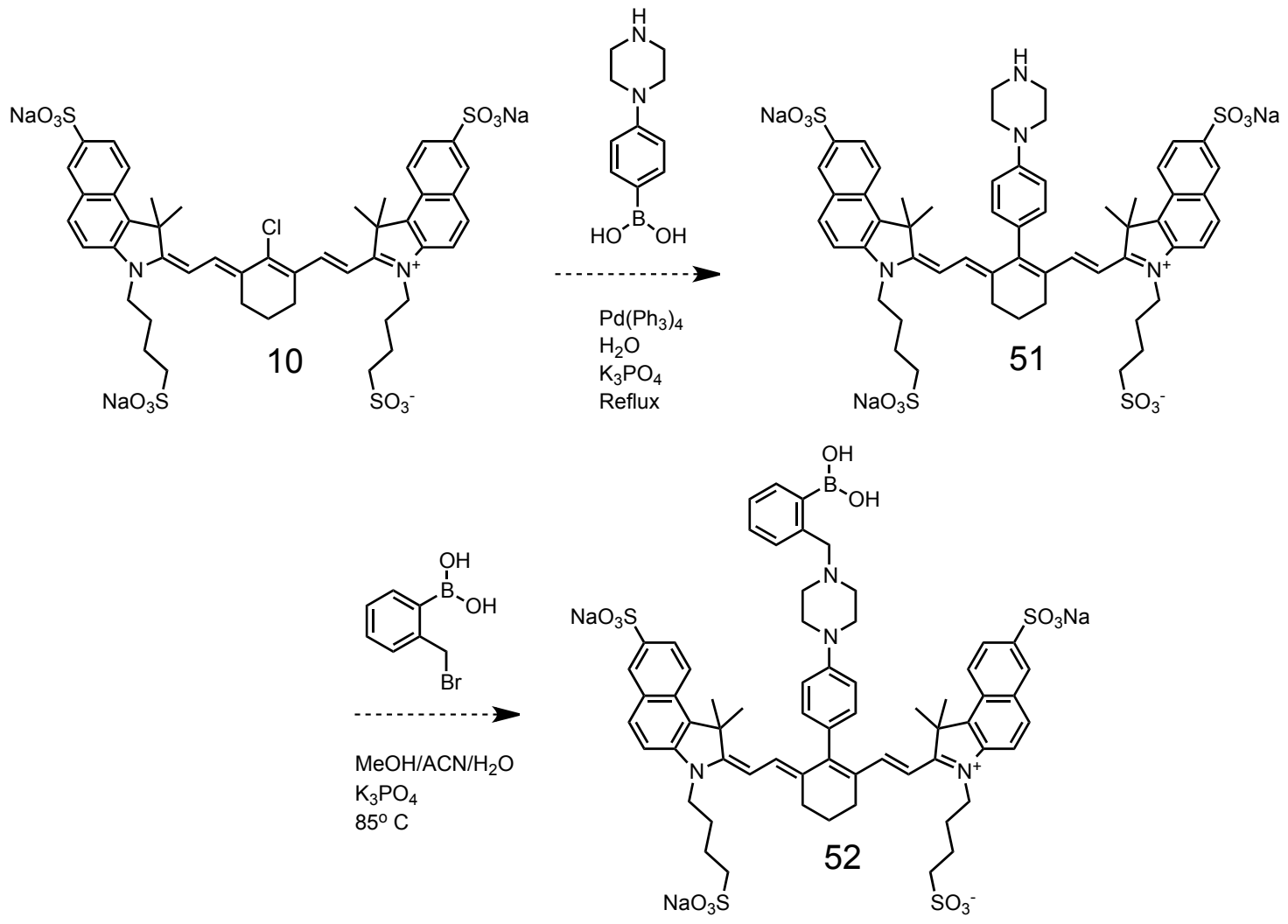

Figure 3.16: Unsuccessful Phenylboronic Acid Compound 52

is assumed that it would improve yields. However, in the cases where the reactions either failed to proceed at all, or produced mixtures of potential product and side product, it is unlikely that refining this aspect of the reaction would alleviate these difficulties.

Better isolation and purification techniques would prove advantageous for the continuation of this work, but aside from the inclusion of SPE, or other similar phase extraction workups, there are few obvious paths forward. Additionally, expansion of the available range of coupling partner types might provide a better understand of the reactivity of $\mathbf{1 0}$. Access to other methods of appending pieces onto $\mathbf{1 0}$ could allow for easier access to bis-boronic acid motifs. Additionally, though Palladium catalysis has 
proved robust and simple, if the hypothesis that some of these reactions failed due to steric bulk at the catalytic center is accurate, attempts at other transition metal catalysts may provide more success. Along those lines, the degradation products of 10 that were observed during both successful and unsuccessful coupling reactions were never observed in the initial attempts at appending amines to $\mathbf{1 0}$ without a catalyst present.

Another note of interest is that despite the considerable difficulty in achieving coupling outside the noted examples, derivatives of $\mathbf{1 0}$ that were achieved provide surprisingly clean H-NMR and C-NMR spectra for a large naphthindole cyanine.

\subsection{Future Work}

The goal of this project is synthesis of a NIR cyanine based glucose sensor. Although that end was not achieved in this work, some progress has been made. 45 indicates that the piperazine moiety appended to the cyanine functions at least in a manner resembling that predicted from the $\mathrm{pH}$ sensitive cyanines. Due to 45 's poor binding, and the difficulty in accessing compounds with which it can be compared, it is unclear how amenable this binding event is to tuning through the addition of extra functional groups, or a second boronic acid.

Some of this is currently being addressed. Efforts to append a tris-secondary amine motif $\mathbf{5 3}$ are currently under way, and attempts at achieving intermediates which provide access to chemistry besides amine alkylation for appending boronic acids are also under way $\mathbf{5 4 .}$

Additionally, cyanine derivatives are not the only NIR fluorophores available for 

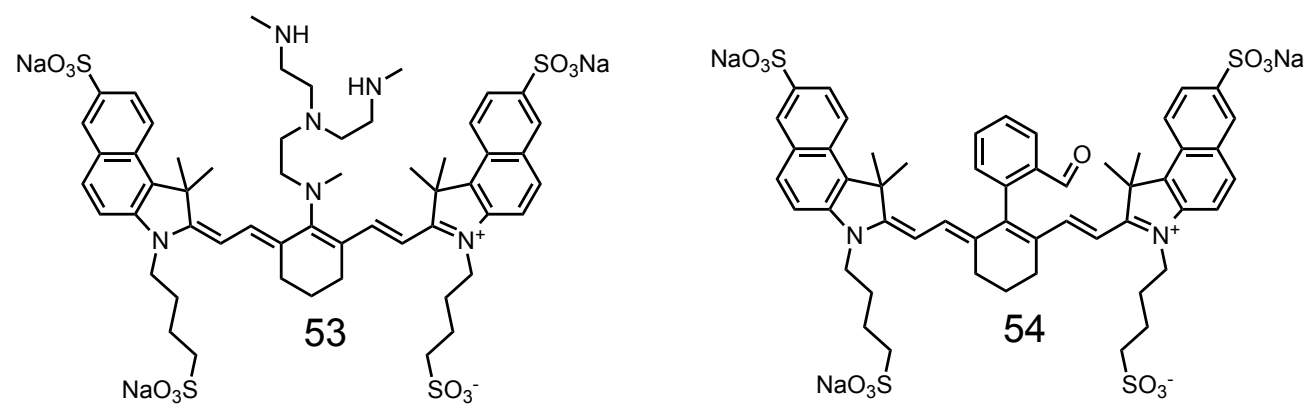

Figure 3.17: Potential Intermediates for achieving a bis boronic acid based receptor.

this type of work, both BODIPY, and Rhodamine based fluorophores present NIR excitation and emission bands that would be acceptable for this type of project. However, like most cyanines, both BODIPY and Rhodamine compounds in general require organic co-solvent additions to be kept soluble [71, 72]. 


\section{Chapter 4}

\section{Erythrocyte Loading}

\subsection{Erythrosensors}

Current methods of monitoring blood glucose levels are accurate but invasive. Under the most popular method, each monitoring event requires a finger prick to obtain a capillary blood sample. Monitoring blood glucose for diabetes mellitus requires frequent monitoring events, or continuous monitoring. As diabetes cases become more advanced or severe, the frequency of monitoring events must increase to enable tighter control of blood glucose levels. Making monitoring events non-invasive presents an opportunity to improve fidelity in patients who may experience negative outcomes should they fail to accurately monitor and control blood glucose levels.

One way that the invasiveness of monitoring can be reduced is through the use of fluorescent sensors circulating in the blood stream that can be interrogated either manually, or automatically with no invasive finger pricks. Foreign small molecules 


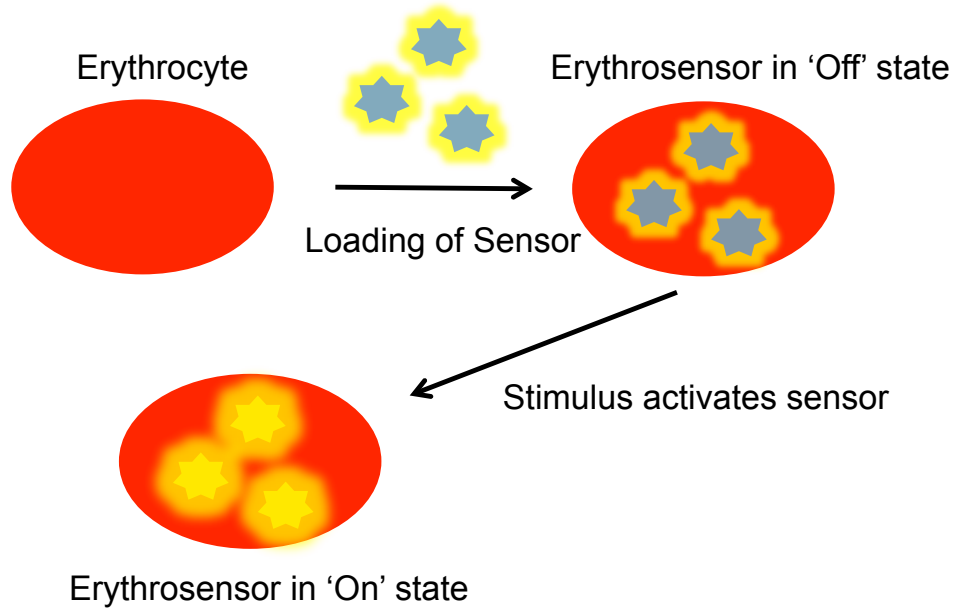

Figure 4.1: Generic Erythrosensor

in the blood stream are cleared quickly by the kidneys, so to improve retention of a fluorescent sensor, the idea of loading into a red blood cell as a carrier was devised. This sensor carrying red blood cell has been colloquially referred to as a Erythrosensor, Figure $4.1[73,74,75]$.

To successfully bind glucose at concentrations relevant to blood sugar perturbations in patients with diabetes mellitus, a potential glucose sensor must bind with a $\mathrm{K}_{\mathrm{d}}$ around 7-10 $\mathrm{mM}$, and would ideally have a significantly weaker $\mathrm{K}_{\mathrm{d}}$ for potential competitors, such as fructose or ascorbic acid.

\subsubsection{Erythrocyte Loading Methods}

Loading of small molecules or proteins into erythrocytes for delivery into humans as therapeutics, or animals for research purposes is well established and clinical trials investigating the efficacy of erythrocytes as drug delivery systems for a variety of compounds is currently underway [76]. Loading of foreign compounds into, or onto 
erythrocytes can be achieved through a variety of means. Currently one of the most frequently utilized methods for loading of cargo molecules is a hypotonic swelling procedure that induces transient pores in the plasma membrane of the treated erythrocytes [77]. Though well characterized, and relatively effective, incubating erythrocytes in hypotonic conditions can have unwanted effects on cell morphology and retention in vivo [78].

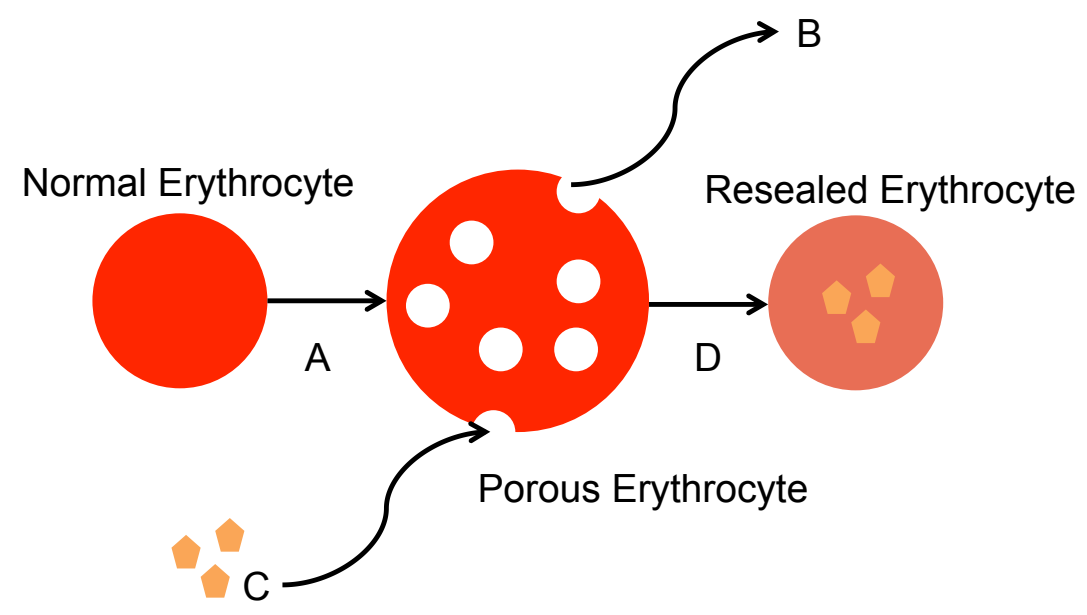

Figure 4.2: A - Hypo-osmotic conditions induce swelling, and pore formation, B Intracellular components diffuse across pores in cell membrane, including Hemoglobin, C - Potential cargo diffuses through membrane pores into erythrocyte, D - Restoration of iso-osmotic conditions reseals erythrocyte, now with depleted Hemoglobin, and carrying cargo.

Due to these detriments, other methods of loading into erythrocytes are currently being pursued. Fusion with liposomes bearing cargos intended for encapsulation in the erythrocyte has been studied, and appears to provide healthier erythrocytes post loading, but as of this writing, has not appeared in clinical trials [79]. Attempts at using cell penetrating peptides bearing cargo for encapsulation have also been explored [80]. 
Loading cargo into the erythrocyte has some advantages, but presents the distinct disadvantage that, whatever the role of the cargo, it must act within the erythrocyte, as once it is encapsulated, diffusion into serum is unlikely. Therefore affixing cargo onto erythrocyte membranes has also been explored. Genetically engineered erythrocytes bearing sites for covalent coupling of cargo have been explored [81, 82]. With the capability to culture erythrocytes ex vivo, engineered erythrocytes are attractive tools for delivery of therapeutics [83].

\subsubsection{Applications}

Erythrocytes as drug delivery systems have been used to deliver dexamethasone sodium phosphate as a therapy for children with Ataxia-telangiectasia, a neurodegenerative genetic disorder [84, 85]. Dexamethasone is a lipophilic steroid, when modified with a charged phosphate group, leakage through membranes is reduced significantly. Overtime the phosphate is hydrolyzed, allowing the dexamethasone to escape from its carrier erythrocyte and into the body, allowing for steady slow release.

Similarly encapsulation of L-Asparaginase into erythrocytes has been explored to improve outcomes in elderly patients suffering from acute lymphoblastic leukemia $[86,87]$. Decreases in serum asparagine has been associated with improvements in patient outcomes, and intravenous treatment with L-asparaginase effectively achieves this in young adults. However in elderly patients, direct intravenous treatment with L-asparaginase leads to negative outcomes. Encapsulation into erythrocytes was attempted to address this issue and trials still appear to be ongoing.

Both of the prior examples rely on using erythrocytes as a system to protect a therapeutic from either quick clearance from serum, or to prevent side effects 


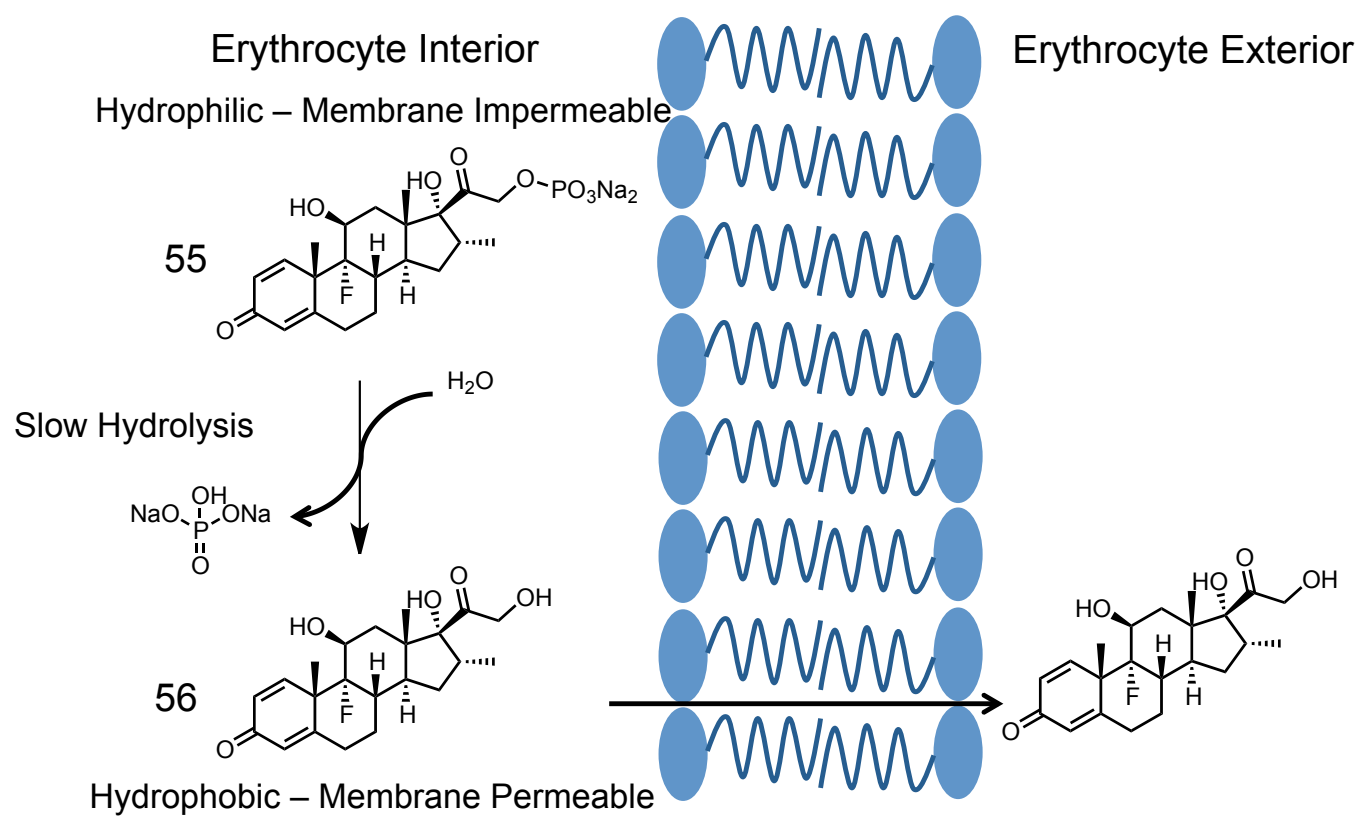

Figure 4.3: Dexamethasone Phosphate hydrolyses slowly in neutral media, allowing controlled release of Dexamethasone from carrier erythrocytes.

from a therapeutic being free in solution. Loading Dexamethasone Phosphate, or L-Asparaginase into the erythrocyte essentially employs the erythrocyte as a trojan horse for the delivery of a steroid therapeutic in the former, and a working enzyme catalyzing chemistry in the latter. Loading substrate onto erythrocyte membranes has also been explored [81] 4.4 .

Engineered erythrocytes expressing a transmembrane protein which possesses an LPXTG motif on the cell exterior serve as a target for Sortase proteins. Sortases selectively join N-terminal glycines to the C-terminal LPXTG motif [88]. As this method covalently and selectively appends cargo to the exterior of erythrocyte, it is therefore limited to cargo which can remain attached to the erythrocyte exterior.

Loading cargo onto erythrocyte cell surfaces can also be performed through erythrocyte specific antibodies, which have an advantage of experimental simplicity, but 


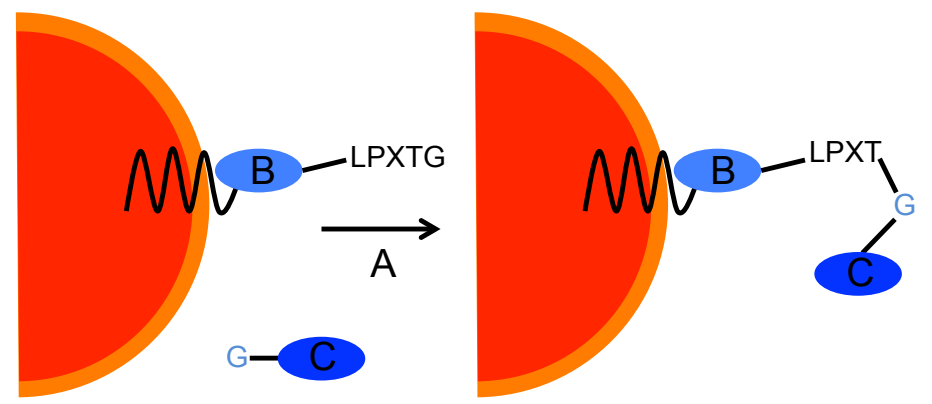

Figure 4.4: A - Sortase proteins target C-terminal LPXTG motifs and append available N-Terminal Glycines in a 2 step sequence, B - Modified transmembrane protein expressing a C-terminal LPXTG motif, C - Intended cargo for attachment, possessing an N-terminal Glycine.

are not attached to the erythrocyte covalently. Lacking a covalent bond to the erythrocyte surface leads to slow dissociation of the antibody, and slow dissociation of the antibody tagged cargo in vivo [89, 90]. If this dissociation is intended, such as for slow, non-specific release of a therapeutic, it is an advantageous feature.

\subsection{Erythrocyte Loading With Tetra-Sulfonated Cya- nine Derivates}

With a significant body of literature describing hypo-osmotic loading of small molecules, and proteins into erythrocytes, it was proposed to determine whether a NIR sensor could be loaded into erythrocytes, injected into a model system, and successfully excited and detected. Collaborators at the University of Swansea had designed a system using a laser diode and silicon detectors, to both excite, and detect, in the NIR, Figures 4.5, 4.6 [74].

Erythrocyte loading through osmotic modulation was determined to be the most accessible procedure for loading of the sensors described herein. Literature on the mor- 


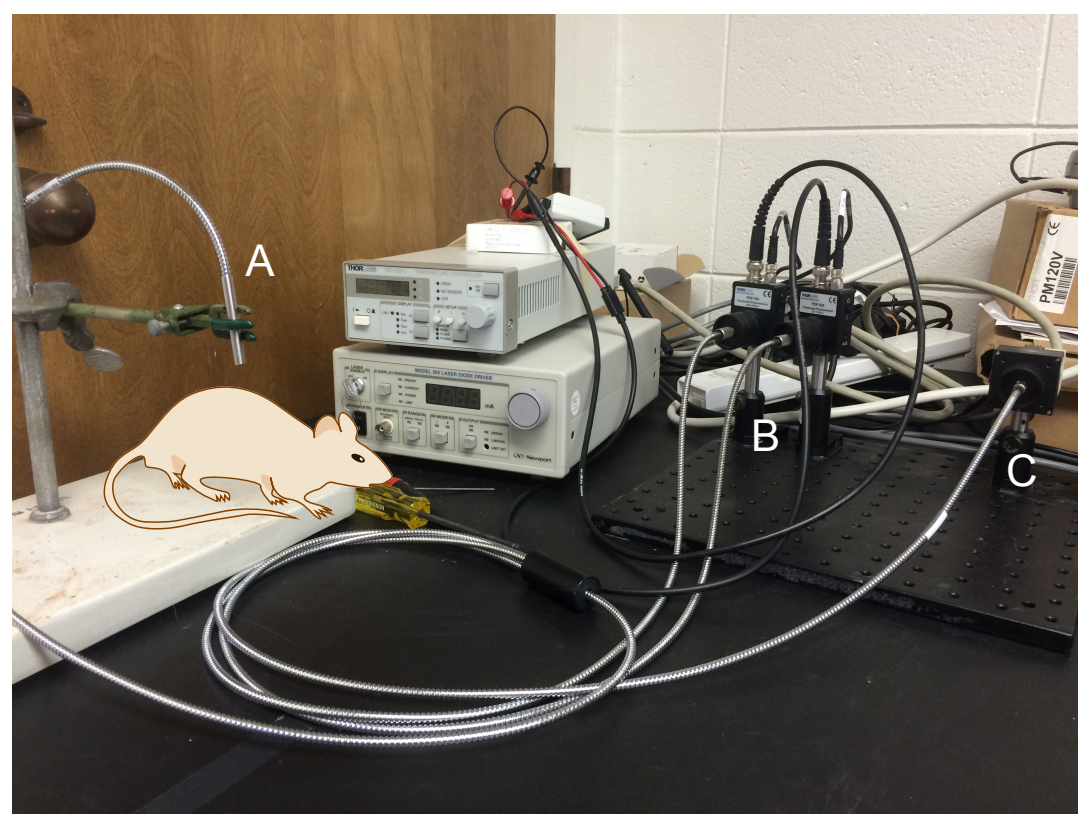

Figure 4.5: A - Probe Head, B - Silicon Detectors equipped with bandpass filters, C - Laser Diode.

phological and physiological changes to erythrocytes that occur during hypo-osmotic loading conditions is diverse. It covers different species, different types of cargo intended for loading, and different methodologies for performing hypo-osmotic loading [91, 92, 93, 94]. The literature surrounding hypo-osmotic loading of compounds into erythrocytes for use in vivo includes both animal model systems, and humans during clinical trials.

This range in model systems has roots in the robustness of erythrocytes of different species to osmotic shock, the robustness of the model animal itself to modulations in whole blood volumes, the easy of surgical access to blood vessels for withdrawl and injection of blood, and the ease of access to healthy, fresh, and sometimes autologous erythrocytes.

For instance, one group at the University of Pittsburg, studying enzyme therapies 


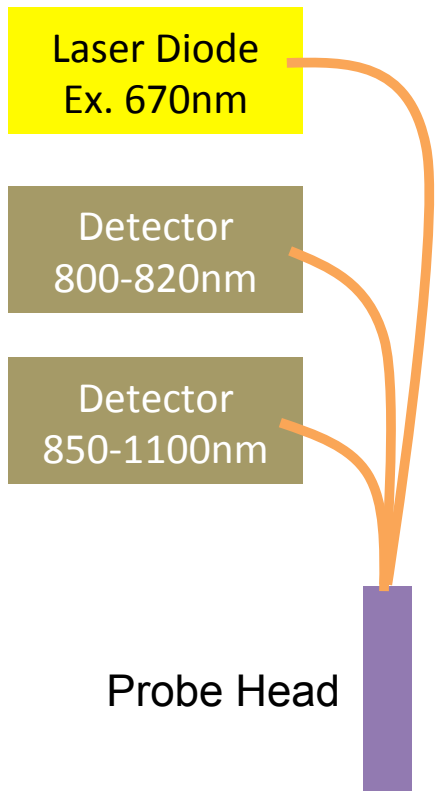

Figure 4.6: Schematic of Instrument for taking Fluorescence Measurements in vivo for Gaucher's Disease, simply withdrew blood from members of the research group for experiments ex vivo [92]. Groups studying acetylcholinesterase (AChE) activity employ erythrocytes from a wide variety of species, as well as human for ex vivo studies, including rat, guinea pig, horse, cow, and rabbit, [95, 96, 97]. As some of this work targets AChE Inhibitors, it would be impractical to perform in vivo. Osmotic loading and modification of erythrocytes to study pathogenesis and resistance mechanisms in Plasmodium sp. and Adenovirus activity, and E. coli interaction with erythrocytes show a preference for performing studies on a range of erythrocytes beyond just human, including rhesus and guinea pig [98, 99, 100].

Two different loading procedures were used over the course of the experiments described herein. A dilution based procedure which produces low-hemoglobin cargo loaded erythrocytes, sometimes referred to as 'ghosts' for their translucent pink color after losing high percentages of hemoglobin, and a dialysis based procedure which 
produced erythrocytes which ideally retained $80 \%$ of original hemoglobin concentrations. Dilution based procedures have the advantage of experimental simplicity, but produce erythrocytes which are often deformed and potentially quickly cleared from circulation by the spleen. Dialysis based procedures which are used clinically, are experimentally more arduous, and were mostly performed ad-hoc in this work, Figure 4.7. At least two companies have designed specialty dialysis instruments for loading small molecules and proteins into erythrocytes for clinical use $[85,84,86]$.

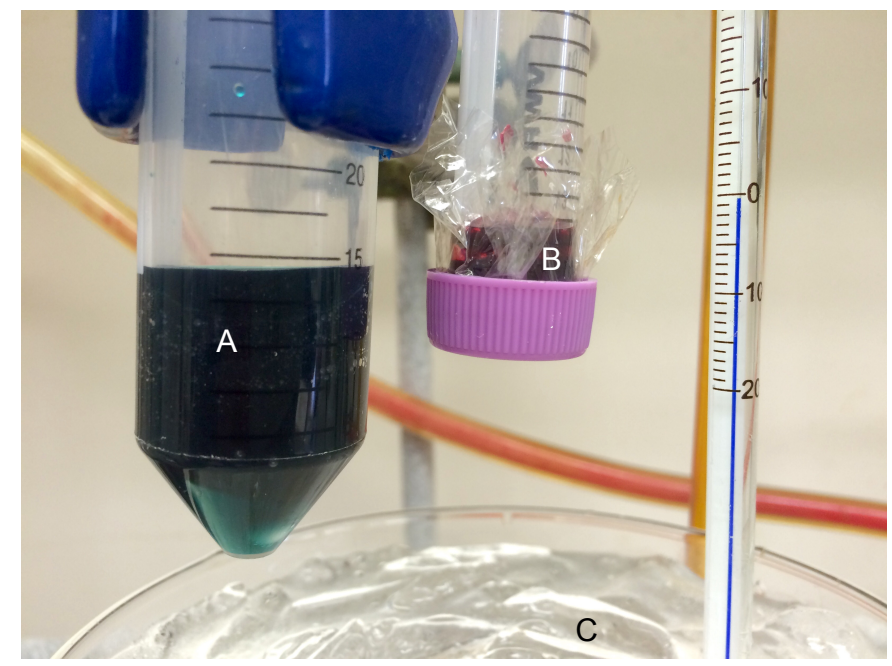

Figure 4.7: Erythrocyte Loading: A - Hypo-osmotic loading solution containing 11, B - Isolated and washed erythrocytes in ad-hoc dialysis chamber, $80 \%$ Hematoctrit, C - Ice Bath.

Loading cargo into erythrocytes using hypo-osmotic conditions which produced hemoglobin deficient erythrocytes is straightforward. Loading buffer $(10 \mathrm{~mL}, 10 \mathrm{mM}$ $\mathrm{NaH}_{2} \mathrm{PO}_{4}, 20 \mathrm{mM}$ glucose, $4 \mathrm{mM} \mathrm{MgCl}_{2}, 3 \mathrm{mM}$ reduced glutathione, $2 \mathrm{mM}$ ATP, and intended cargo, $\mathrm{pH}=7.4$ ) was cooled to between $0-4^{\circ} \mathrm{C}$, and $1 \mathrm{~mL}$ of isolated, washed, and pelleted erythrocytes were added. The mixture was allowed to stir for 45 minutes, and then returned to iso-osmotic conditions through slow addition of 
resealing buffer $\left(1.1 \mathrm{~mL}, 35 \mathrm{mM} \mathrm{Na} \mathrm{HPO}_{4}, 1.606 \mathrm{M} \mathrm{KCl}, 194 \mathrm{mM} \mathrm{NaCl}, 4 \mathrm{mM}\right.$ $\mathrm{MgCl}_{2}, 100 \mathrm{mM}$ glucose, $2 \mathrm{mM}$ ATP, $\mathrm{pH}=7.4$ ), and kept in ice bath for additional 20 minutes. The solution was then moved to a $37^{\circ} \mathrm{C}$ oil bath, and diluted with wash buffer (7.9 mL, $10 \mathrm{mM}$ HEPES, $140 \mathrm{mM} \mathrm{NaCl}, 5 \mathrm{mM}$ glucose, $\mathrm{pH}=7.4)$, and allowed to stir for additional 30 minutes. Resealed erythrocytes were then isolated, pelleted, and resuspended in 1x PBS, or wash buffer for use. Throughout the literature there is little variation on this procedure except for some groups preferring to use $10 \mathrm{x}$ PBS $(1.65 \mathrm{M})$ and $0.1 \mathrm{x}$ PBS $(16.5 \mathrm{mM})$ in favor of the resealing and loading buffers, respectively.

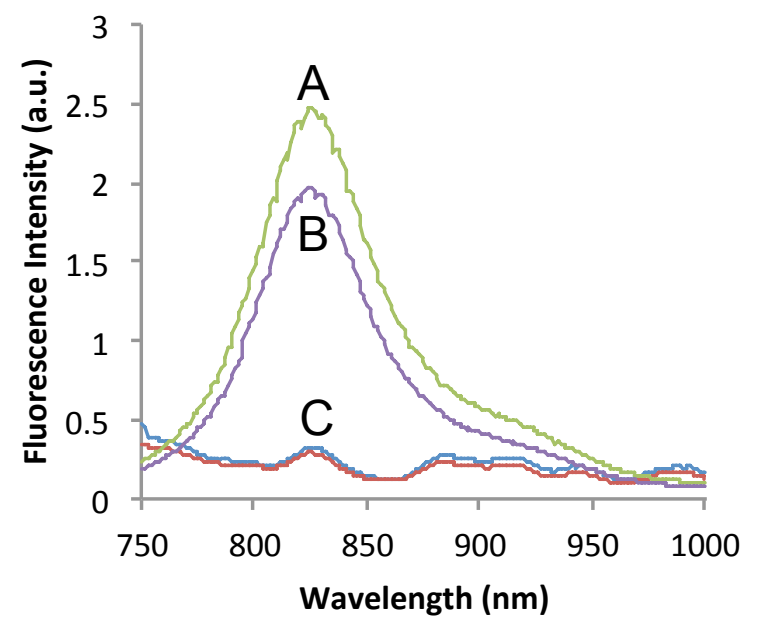

Figure 4.8: A - 10\% hematocrit horse erythrocytes loaded with $\mathbf{1 1}$ under low hemoglobin loading conditions, B- $5 \%$ hematocrit horse erythrocytes loaded with 11 under low hemoglobin loading conditions, $\mathrm{C}-10 \%$ and $5 \%$ hematocrit horse erythrocytes, all dilutions in $165 \mathrm{mM}$ PBS, at $\mathrm{pH} 7.4, \lambda$ ex: $670 \mathrm{~nm}$, slits $15 \mathrm{~nm} / 15 \mathrm{~nm}$.

Dilution based loading provide a clear fluorescence output indicating presence of the loaded sensor, Figure 4.8. Erythrocytes loaded with Sensor 11 can even be subjected to $\mathrm{pH}$ titrations, where it appears that the sensor provides the same $\mathrm{pK}_{\mathrm{a}}$ 
as it does in bulk solution titrations, Figure 4.9.

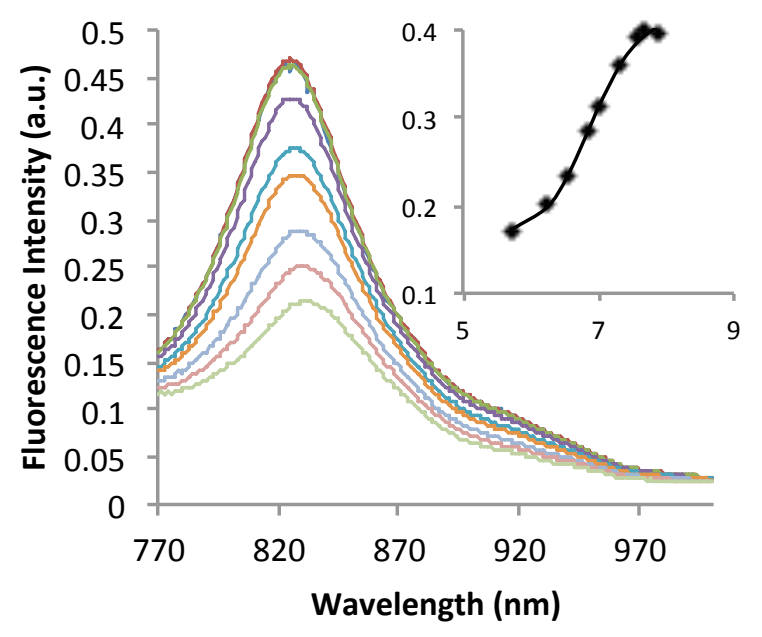

Figure 4.9: Erythrocytes loaded with Sensor $\mathbf{1 1}$ titrated from basic to acidic conditions with aliqouts of $1 \mathrm{M} \mathrm{HCl}$. Erythrocytes were loaded with dilution loading procedure, and diluted to $5 \%$ hematocrit. $\lambda$ ex: $670 \mathrm{~nm}$, slits $15 \mathrm{~nm} / 15 \mathrm{~nm}$, inset is fluorescence intensity at $810 \mathrm{~nm}$. Final concentration of Sensor $\mathbf{1 1}$ in erythrocytes was $18.1 \mu \mathrm{M}$, and final concentration in cuvette is $0.9 \mathrm{nM}, \mathrm{pK}_{\mathrm{a}}$ was determined to be 6.9.

In order to gain some measure of the robustness of sensor loaded erythrocytes, they were subjected to an extended series of the same washing and centrifugation steps required to isolate erythrocytes pre-loading procedure, and post-loading, Figure 4.10. The fluorescence signal present in the supernatants of these wash steps could indicate that either Sensor $\mathbf{1 1}$ is not fully internalized into the erythrocytes, and is being washed off during these step. Or that the treated erythrocytes are fragile to these handling steps post treatment. Neither option is ideal, and the latter is more likely.

Loading cargo into erythrocytes using hypo-osmotic conditions which produce normal hemoglobin erythrocytes is similar, but less straight forward. Instead of direct dilution of erythrocytes into the Loading Buffer, they are added to the buffer in an inner dialysis chamber of a two chamber apparatus. As pelleted erythrocytes 


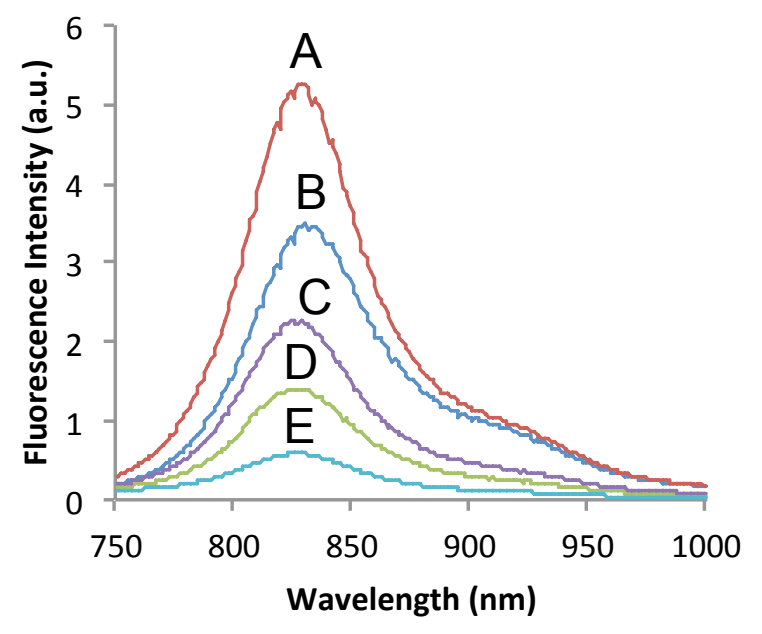

Figure 4.10: Fluorometer scans of supernatants collected from separate washing steps of the dilution loading process, and from washing steps after completing the loading procedure. A - Supernatant from first wash step following loading, B - Supernatant from second wash step following loading, C - Supernatant from third wash step following loading, D - supernatant of erythrocyte incubation in $165 \mathrm{mM}$ PBS for $1 \mathrm{hr}, \mathrm{E}$ - supernatant of erythrocyte incubation in $165 \mathrm{mM}$ PBS for an additional hour, $\lambda$ ex: $670 \mathrm{~nm}$, slits $15 \mathrm{~nm} / 15 \mathrm{~nm}$.

are relatively viscous, this requires that both the loading buffer, and the dialysis chamber have some mechanism of gentle stirring. The whole solution is returned to iso-osmotic conditions through adding the Resealing Buffer to the outer chamber, and the temperature variations are the same as for the prior procedure.

Healthy erythrocytes even at low hematocrit concentrations are highly scattering. Figure 4.11 shows the results of fluorometer scans of sensor $\mathbf{1 1}$ loaded into erythrocytes via the dialysis procedure. Little difference between the erythrocytes subjected to loading, and control erythrocytes could be determined. Though there are differences in the collected spectra, it is not clear whether direct comparison of the fluorescence scattering can provide clear indication of presence of $\mathbf{1 1}$ or just 


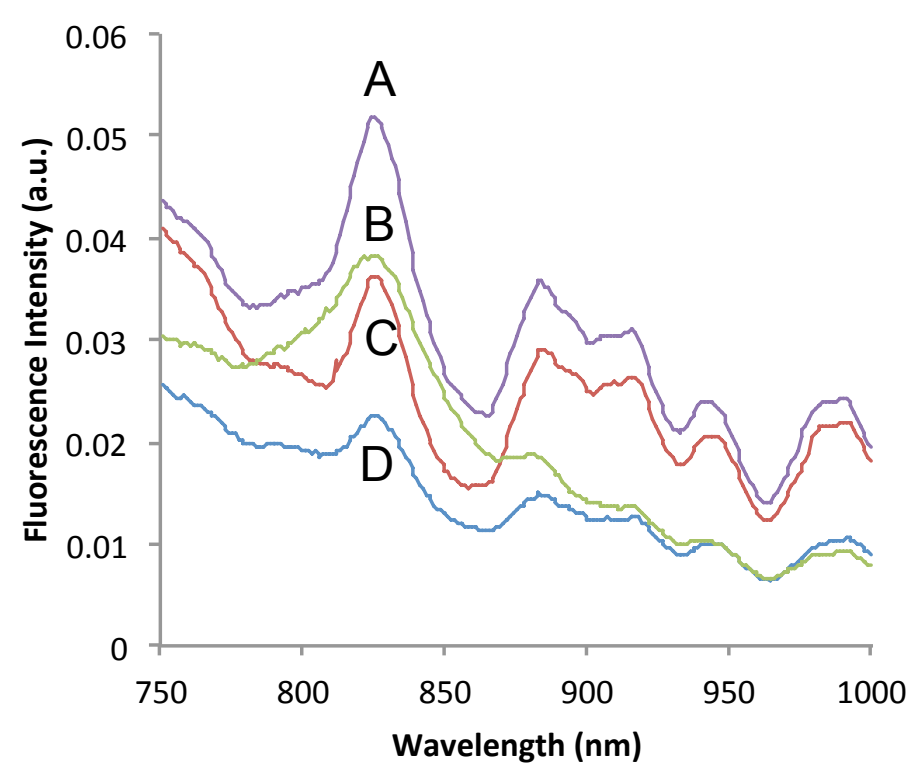

Figure 4.11: A - 10\% hematocrit horse erythrocytes, B- 10\% hematocrit horse erythrocytes loaded with 11, C - 5\% hematocrit horse erythrocytes, D - 5\% hematocrit horse erythrocytes loaded with 11, all dilutions in $165 \mathrm{mM} \mathrm{PBS,} \mathrm{at} \mathrm{pH} \mathrm{7.4,} \lambda$ ex: 670nm (slits 15nm/15nm).

morphological changes from the osmotic stress the erythrocyte was subjected to.

Even though erythrocytes obtained from dialysis loading only provide scattering, lysis of these same erythrocytes via repeated freeze/thaw cycles rescues fluorescence output in the supernatant of the freeze thaw experiment, Figure 4.12 .

Another necessary control is determining the stability of the loaded erythrocytes, as Figure 4.10 indicates post-dilution loading, the cells appear fragile. However, subjecting dialysis-loaded erythrocytes to these same experiments provides little indication of this same phenomenon, Figure 4.13.

Meissner and Ritter's instrument was used in a set of cuvette experiments, with the probe head being directed at a cuvette containing diluted erythrocytes. Signal output indicates that the presence of sensor $\mathbf{1 1}$ in the dialysis loaded erythrocytes 


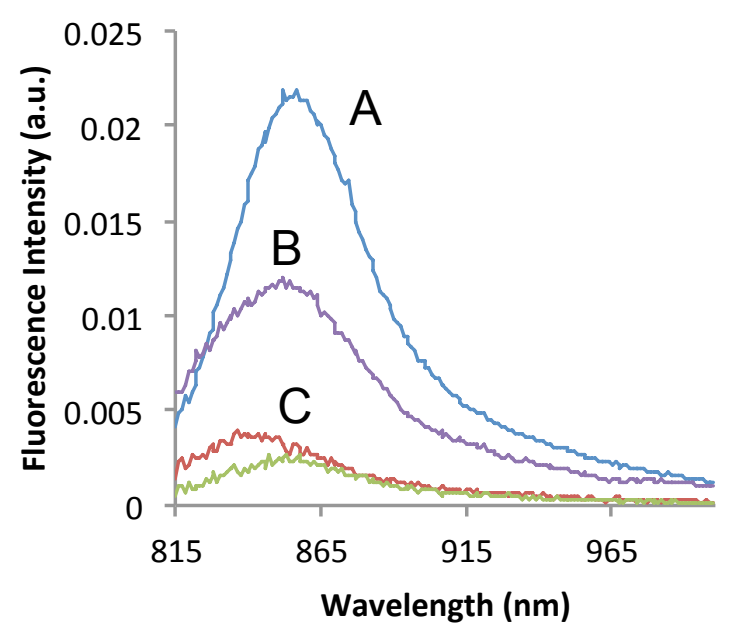

Figure 4.12: Fluorescence detected from the supernatant of freez/thaw lysis of dialysis loaded erythrocytes. A - Supernatant from first wash step following loading, B Signal from Freeze/Thaw Lysis of loaded erythrocytes, C - Supernatant from second and third wash step following loading, $\lambda$ ex: 670nm, slits 15nm/15nm.

is likely, Figure 4.14. This set of experiments, though providing assurance that 11 is loaded in the erythrocytes, does not provide a measure of the effectiveness of the loading. It is likely that the cuvette experiment used to produce Figure 4.14 could be modified to provide clearer data. Combined, the laser and fluorometer experiments provide qualitative indication that the dialysis loading is successful.

All of these experiments were performed using horse blood, for cost and safety reasons. The dialysis and dilution loading procedures of Sensor $\mathbf{1 1}$ as shown in Figures $4.8,4.10,4.11,4.13,4.12$, and 4.14 were designed to provide a concentration of $55 \mu \mathrm{M}$ in the erythrocyte interior. This interior concentration, when diluted by hematocrit concentration in the cuvette should give final concentrations of $5.5 \mu \mathrm{M}$ and $2.75 \mu \mathrm{M}$ for the $10 \%$ and $5 \%$ hematocrit scans, respectively.

An advantageous direction for this project to take following this work is the es- 


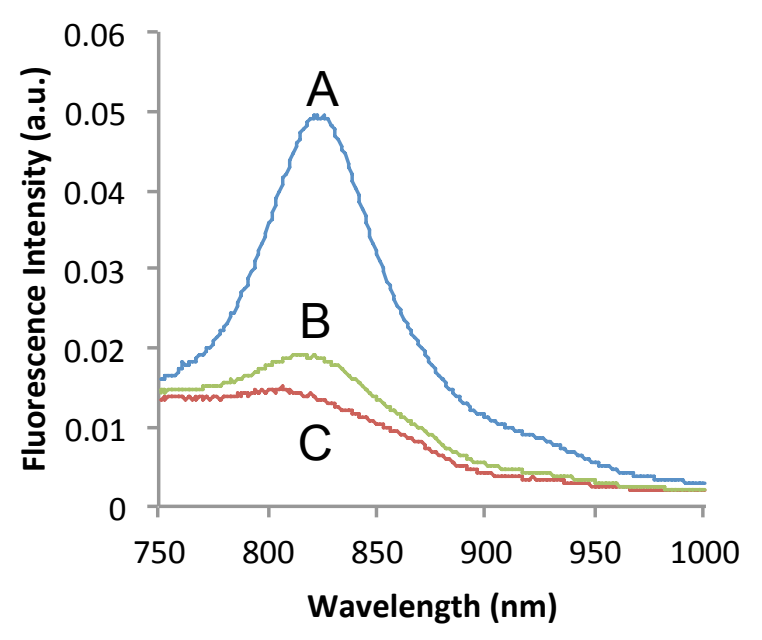

Figure 4.13: Fluorescence detected from the supernatant of iterative was steps for dialysis loaded erythrocytes. A - Supernatant from first wash step following loading, B - Supernatant from third wash step following loading, C - Supernatant from second wash step following loading, $\lambda$ ex: $670 \mathrm{~nm}$, slits $15 \mathrm{~nm} / 15 \mathrm{~nm}$.

tablishment of a system determine erythrocyte loading and efficiency. The inability to provide a standard curve for erythrocyte loading and health due to lack of appropriate microscopy tools was a significant hindrance on the further studies described herein. 


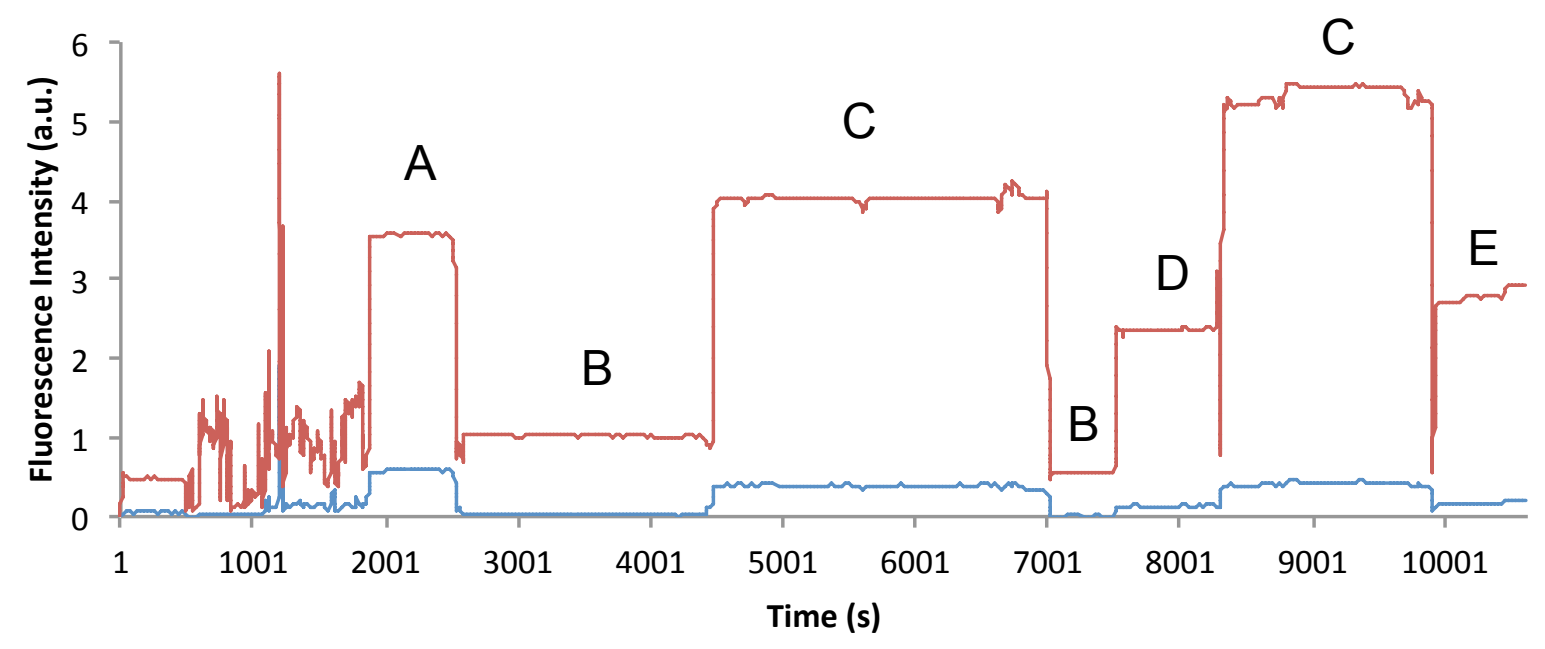

Figure 4.14: Instrument measures of dialysis loaded, and control erythrocytes. $\lambda$ ex: at $670 \mathrm{~nm}$, top channel $800 \mathrm{~nm}-820 \mathrm{~nm}$, bottom channel $850 \mathrm{~nm}-1000 \mathrm{~nm}$, all dilutions in $165 \mathrm{mM}$ PBS, $\mathrm{pH}=7.4, \mathrm{~A}-10 \%$ hematocrit erythrocytes loaded with 11, B Control, Self-Masking Cuvette, C - 5\% hematocrit erythrocytes loaded with 11, D $10 \%$ hematocrit control erythrocytes, E- 5\%hematocrit control erythrocytes.

\subsubsection{Animal Studies}

Rats were used as the model system for the in vivo portion of this work. Using the Dialysis produces described previously, rat erythrocytes were loaded with sensor $\mathbf{1 1}$ and resuspended in 1x PBS to 50\% hematocrit for injection. It was proposed that an injection of sensor loaded erythrocytes would provide a steady signal over time, as opposed to an injection of free dye in solution, which would be quickly cleared from serum. If persistent and steady signal could be achieved, serum conditions could be modulated to induce changes in $\mathrm{pH}$ which could be observed by fluorescence with the instrument designed by collaborators Meissner and Ritter.

Adult male Sprague-Dawley rats were anesthetized with isofluorane and a catheter was inserted into the jugular vein. A small portion of the abdomen was shaved and 
the the probe head of the instrument was immobilized over the shaved skin. A $200 \mu \mathrm{L}$ injection of either aqueous sensor $\mathbf{1 1}$ at $250 \mu \mathrm{M}$, or erythrocytes loaded with sensor 11 at an assumed concentration of $125 \mu \mathrm{M}$ on the erythrocyte interior was performed, followed by an injection of $100 \mu \mathrm{L}$ heparin. Fluorescence intensity from the injection was collected at the probe head, and processed by software written by Meissner and Ritter.

With reasonable assurance that $\mathbf{1 1}$ was loaded into erythrocytes using the dialysis loading procedure, in vivo experiments were performed on anesthetized rats, Figures 4.16, 4.17. The injection of erythrocytes loaded with $\mathbf{1 1}$ into an animal model was expected to give an initial rise in fluorescence as the dye diffuses into the vascular system, followed by an exponential decay as it is cleared from circulation.

$$
N(t)=N_{0} e^{-\lambda t}
$$

It was expected that after initial injection of the sensor loaded erythrocytes fluorescence signal detected would spike substantially, and then begin to decay much more slowly than the free dye. Erythrocytes successfully loaded with sensor 11, if healthy enough to preclude clearance by the spleen would provide a decay in fluorescence at a substantially slower rate than signal from an aqueous injections of $\mathbf{1 1}$ used as a control experiment. Signal decay was expected to follow an exponential decay equation, Figure 4.15, Equation 4.1.

In exponential decay curves initial intensity decays at an exponential rate, and can be described through a time constant $(\tau)$, which is the reciprocal of the rate constant for the decay of the signal. So for the generic decay function described in Figure 4.15, which has a rate constant of $.05 \mathrm{~s}^{-1}, \tau$ is $20 \mathrm{~s}$. 


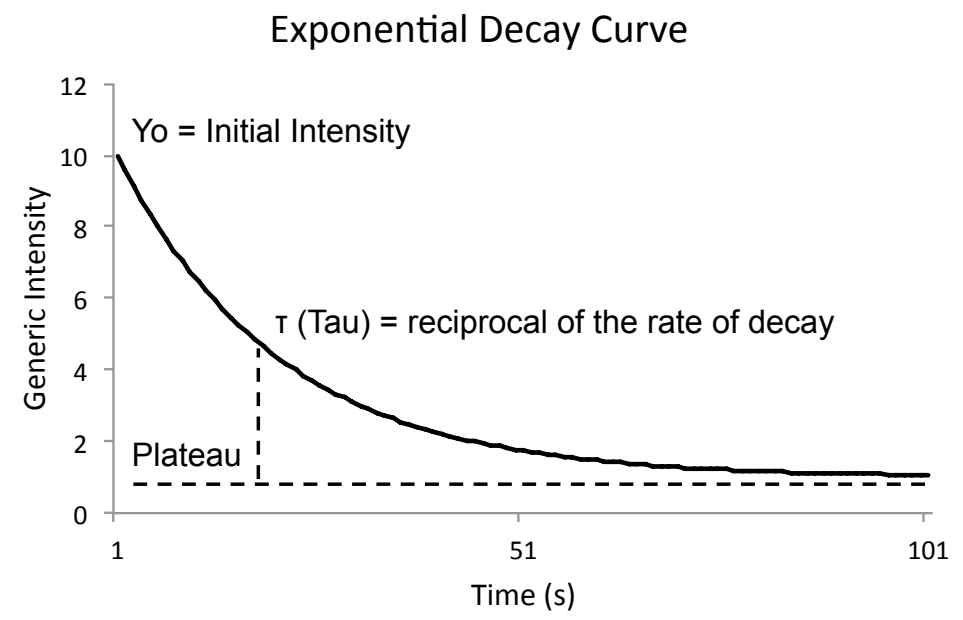

Figure 4.15: A generic exponential decay curve.

Figure 4.16 describes a time course experiment where aqueous 11 was injected into a subject rat, and allowed to clear, followed by an injection of erythrocytes loaded with sensor 11. A similar experiment in reverse order is described in Figure 4.17. In vivo experiments were performed in this manner due in part to the potential difference in serum clearance rates between individual animals. Clearance rates for both aqueous 11 and 11 loaded into erythrocytes were then fit to a double exponential decay curve to approximate signal retention in vivo.

Fits of the collected data indicate that signal from erythrocytes loaded with sensor 11 was retained for roughly double the amount of time as signal from aqueous 11 injected in buffer, in at least one animal, in another animal the time constants of both injections are relatively close in value, while in the remaining two animals, the time constant for the injection of aqueous $\mathbf{1 1}$ indicates longer retention of signal than observed in the erythrocyte injections. Overall a few trends are observable, the first injection into the animal always provided longer time constants, in some cases 


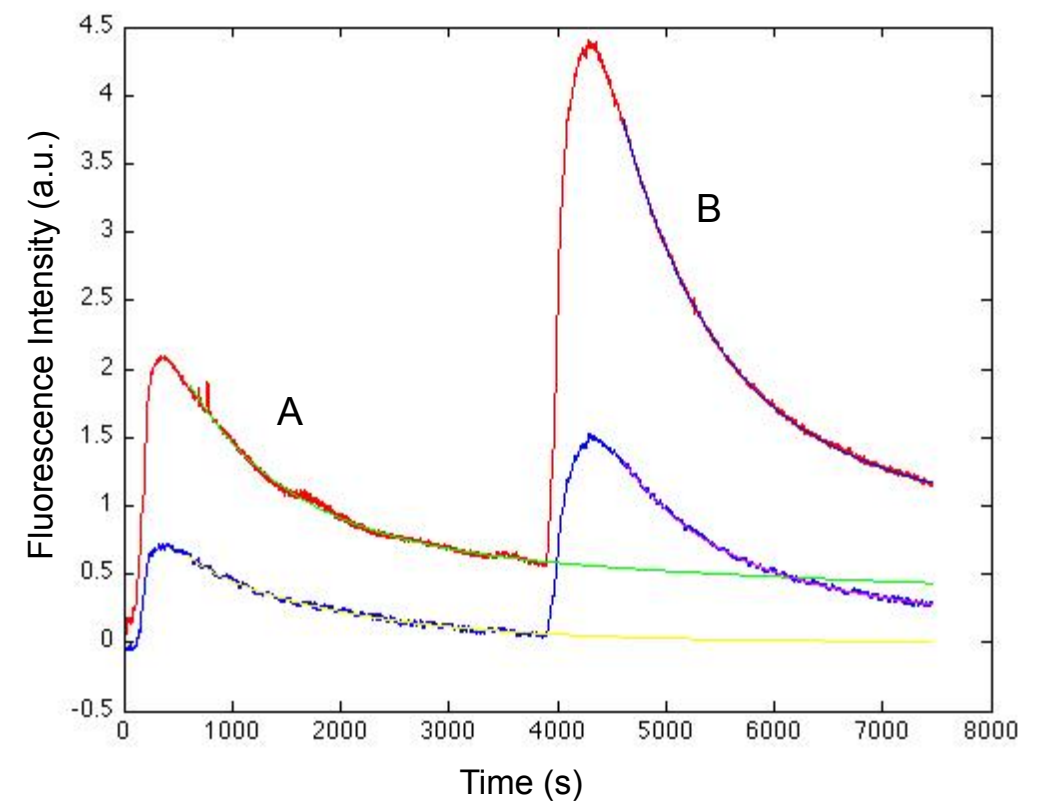

Figure 4.16: A - Injection of free 11, B - Injection of $\mathbf{1 1}$ loaded into Erythrocytes. significantly longer. Also in rats where erythrocytes carrying $\mathbf{1 1}$ were injected first, the time constants for both injections were significantly closer in value than when injection order was reversed.

The data collected from these experiments indicates that the loading procedures used produce erythrocytes that are most likely cleared quickly by the spleen. Additionally, as sensor $\mathbf{1 1}$ is too red to be observed on the fluorescent microscopes available for this work, and controls to determine loading efficiency could not be elucidated, the quantity of $\mathbf{1 1}$ trapped inside the injected erythrocytes remains an unknown variable.

It is also unclear whether the loaded erythrocytes were healthy enough to pass through the spleen without triggering clearance mechanisms. Some evidence from SEM images obtained of loaded cells indicates that the erythrocytes are not presenting uniform morphology post-loading, which may indicate that are not healthy enough 


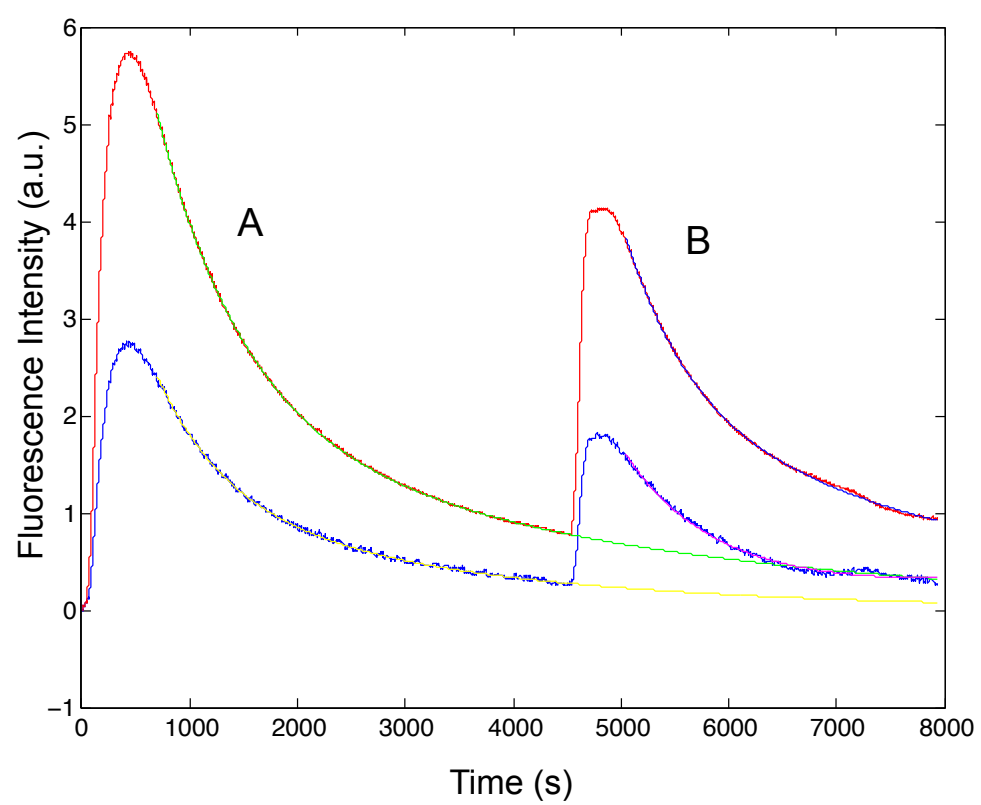

Figure 4.17: A - Injection of $\mathbf{1 1}$ loaded into Erythrocytes, B - Injection of free $\mathbf{1 1 .}$

to be maintained in circulation, Figure 4.18. The mixture of apparently morphologically normal, toroidal erythrocytes, alongside semi-spherical, and spiny erythrocytes indicates that loading may damage the cells, it also solicits the potential that effects of the dialysis may not be uniform. Spiny shaped erythrocytes are indicative of exposure to hyper osmotic conditions, causing the cell to contract significantly, this is not unexpected, considering that resealing of the erythrocytes requires addition of a hyper-osmotic solution to reestablish iso-osmotic conditions.

The resealing step is allowed to stir for an extended time to allow for mixing between the two dialysis chambers. The presence of erythrocytes that appear to have been isolated from a hyper-osmotic environment indicates that mixing between the two chambers may not be occurring rapidly enough to equilibrate over the course of the resealing, or that the high hematocrit solution within the dialysis chamber are 
Time Constants for Signal Decay Following Injection Into Rat Model

\begin{tabular}{|c|c|c|}
\cline { 2 - 3 } \multicolumn{1}{c|}{} & Aqueous 11 & Erythrocyte Loaded 11 \\
\hline $\begin{array}{c}\text { Rat 1 - Erythrocytes } \\
\text { First }\end{array}$ & $4063 \mathrm{~s}$ & $8848 \mathrm{~s}$ \\
\hline $\begin{array}{c}\text { Rat 2 - Erythrocytes } \\
\text { First }\end{array}$ & $3269 \mathrm{~s}$ & $4000 \mathrm{~s}$ \\
\hline $\begin{array}{c}\text { Rat 3 - Free Dye } \\
\text { First }\end{array}$ & $14816 \mathrm{~s}$ & $7914 \mathrm{~s}$ \\
\hline $\begin{array}{c}\text { Rat 4 - Free Dye } \\
\text { First }\end{array}$ & $17803 \mathrm{~s}$ & $5766 \mathrm{~s}$ \\
\hline \begin{tabular}{c} 
Average $\tau$ \\
\hline
\end{tabular} & $9987 \mathrm{~s}+/-7407 \mathrm{~s}$ & $6632 \mathrm{~s}+/-2178 \mathrm{~s}$ \\
\hline
\end{tabular}

Table 4.1: Time Constants for injections of free, aqueous 11, and injections of erythrocytes loaded with 11.

not being uniformly exposed to the changes in osmotic pressure.

\subsubsection{Future Work}

This project has challenges to both the physiology and chemistry aspects of this work. There are however, plenty of experimental avenues to pursue to address the challenges met. Working with rat and horse erythrocytes might be one source of complications in the issues encountered with hypo-osmotic loading. The only directly correlative work published prior to this involved encapsulation of ICG into human, rhesus macaque, and rabbit erythrocytes, wherein work performed here was either with rat, or horse erythrocytes, used for both cost and safety concerns [101]. Further, exploration of erythrocyte loading procedures beyond those currently utilized may be advantageous. Drug delivery system research has pursued the use of erythrocytes, or erythrocyte mimics as delivery mechanisms for quiet some time, with mixed results 

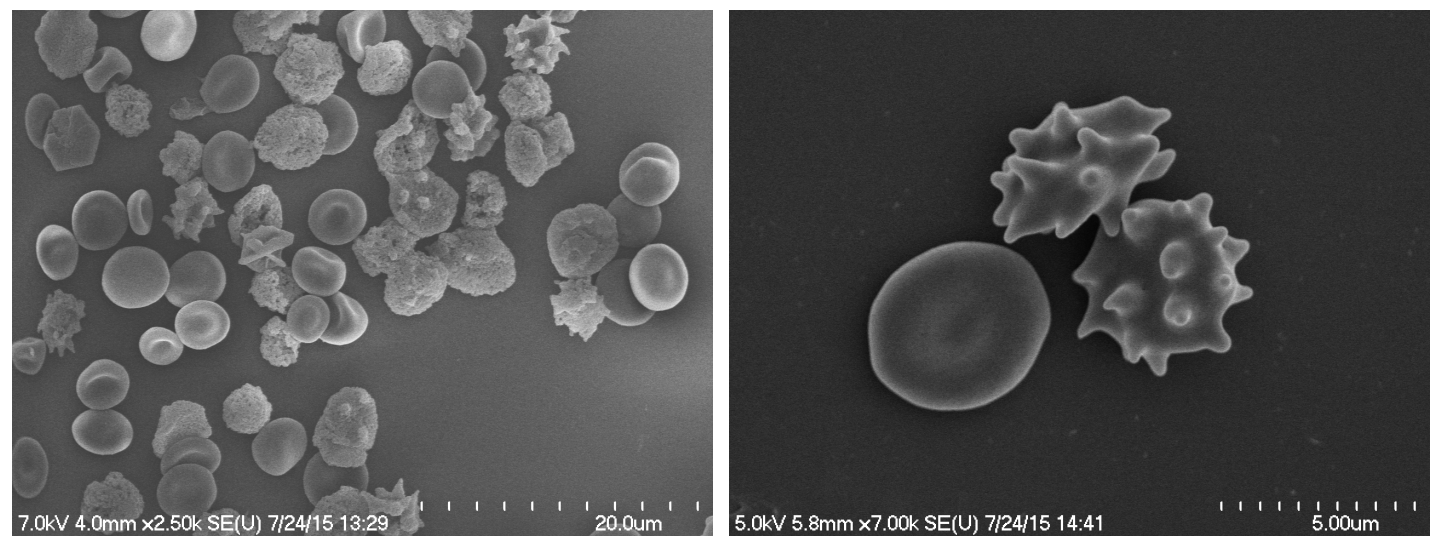

Figure 4.18: SEM Images of Erythrocytes following hypo-osmotic loading of $\mathbf{1 1 .}$ [102].

Designing a glucose sensor with applicable properties for use in vivo presents a unique set of challenges, and the approach taken here was to address solubility in aqueous systems, and emission and excitation wavelengths. Compounds designed and proposed herein provide rudimentary solutions to excitation and emission challenges in vivo, and the solubility issues usually associated with large highly conjugated fluorophores. However, synthetically it appears unclear whether the precursor compound 10 is synthetically amenable to the conceivable chemistry necessary to append two boronic acids to it in a manner capable of providing fluorescence changes similar to those observed in this work.

Another potential route to constructing a successful erythrosensor could be to pursue a bioconjugate approach. Tethering fluorophores or similar small molecules to proteins is well established, but can be difficult to achieve with strict selectivity if attempted in vivo [103]. This selectively concern can be addressed through the use of engineered proteins expressing selective tags for specific chemical functional groups [104, 81]. Conveniently, erythrocytes can be cultured, and modified ex vivo 
making much of this work, relatively accessible, if literature is to be taken at face value [83, 105]. 


\section{Appendix A}

\section{Experimental Procedures and Characterization Data}

\section{A.1 Experimental Procedures}

General Procedures: Solvents were purchased from either Acros or Sigma Aldrich and used as is, with the exception of Acetonitrile and TEA were distilled over calcium hydride before use. Reagents were purchased from either Acros or Sigma Aldrich with the following exceptions: all reagents containing boron were purchased from Combi-Blocks (San Diego, CA), and compound 10 was purchased and used as is from FEW Chemicals (Bitterfeld-Wolfen, Germany). Reactions were monitored by absorbance spectroscopy for shifts in major absorbance bands to determine reaction progress and completion. Products were purified via preparative scale HPLC. Purity was determined by H- and C-NMR spectroscopy along with High Resolution Mass Spectrometry (COSMIC Facility, Old Dominion University). 
HPLC Procedures: A Varian Prostar 335 detector with 2 Varian Prepstar 218 pumps, equipped with a 19x300mm Waters Nova-Pak C18 column was used for HPLC all HPLC purifications. All purifications were performed starting at $99 \% \mathrm{H}_{2} \mathrm{O}: 1 \% \mathrm{ACN}$ running either an approximately $1 \% /$ minute gradient to $70 \% \mathrm{H}_{2} \mathrm{O}: 30 \% \mathrm{ACN}$, or a $1 \% /$ minute gradient to $69 \% \mathrm{H}_{2} \mathrm{O}: 31 \% \mathrm{ACN}$ over 30 minutes. Full absorbance spectrum was used to identify elutions for isolation.

Titrations: All titrations were performed in $165 \mathrm{mM}$ Phosphate Buffered Saline (Cold Spring Harbor Protocol). Solid compound isolated from the HPLC was dissolved in neutral PBS and diluted to desired concentrations and pHs. $\mathrm{pK}_{\mathrm{a}}$ titrations were performed by modulating $\mathrm{pH}$ via aliquots of $1 \mathrm{M} \mathrm{NaOH}$ or $1 \mathrm{M} \mathrm{HCl}$. Sugar binding titrations were performed by formulating Sensor solutions in buffer, at the desired concentrations. Analyte solutions were prepared using the sensor solution so that the overall sensor concentration remains constant throughout the titration.

$\mathrm{pK}_{\mathrm{a}}$ Determination: Fluorescence Intensity measurements, correlated to the $\mathrm{pH}$ of the buffer at which they were collected were plotted and fit against Equation A.1. Where FLInt ${ }_{\min }$ and FLInt ${ }_{\max }$ were minimum and maximum fluorescence intensity values, respectively, and the Hill Coefficient is a function describing cooperativity of interaction, or the velocity at which the slope of the sigmoid changes.

$$
y=\text { FLInt }_{\text {Min }}+\frac{\text { FLInt }_{\text {Min }}-\text { FLInt }_{\text {Min }}}{1+{\frac{x}{p K_{a}}}^{- \text {HillCoefficient }}}
$$

Binding Constant Determination: Fluorescence Intensity measurements were scaled to the fluorescence intensity of the Sensor solution, and reported as I/ $I_{0}$ (Intensity/Original Intensity). Data fits were determined using $\mathrm{I} / \mathrm{I}_{0}$ so that the the fitted equation describing the binding isotherm passes through the origin. Fluorescence 
collected from additions of the Analyte solution were normalized and fit to Equation A.2. Where $B_{\text {Max }}$ describes the maximum fluorescence intensity, and the saturation of the sensor, while $\mathrm{K}_{\mathrm{d}}$ describes the half saturation of the sensor.

$$
y=\frac{B_{M a x} \times x}{K_{d}+x}
$$

Exponential Decay Determination: Fluorescence signal in in vivo experiments were fit to a double exponential decay, A.3.

$$
y=\text { plateau }+ \text { SpanFast } \times \exp \left(-K_{\text {Fast }} \times x\right)+\text { SpanSlow } \times \exp \left(-K_{\text {Slow }} \times x\right)
$$

Span Operators refer to Y-value ranges of the total decay, A.4.

$$
\begin{array}{r}
\text { SpanFast }=(\text { InitialIntensity }- \text { Plateau }) \times(\text { PercentFast }) \\
\text { SpanSlow }=(\text { InitialIntensity }- \text { Plateau }) \times(1-\text { PercentFast })
\end{array}
$$

Initial intensity was determined arbitrarily following initial transients, and once the fluorescence intensity signal started to display an exponential decay. Plateau was assumed to be zero, representing complete decay of signal.

Animal Studies: Adult male Sprague-Dawley rats were anesthetized with isofluorane and a catheter was inserted into the jugular vein. A small portion of the abdomen was shaved and the the probe head of the instrument was immobilized over the shaved skin. A $200 \mu \mathrm{L}$ injection of either aqueous dye at $250 \mu \mathrm{M}$, or dye loaded erythrocytes at an assumed concentration of $125 \mu \mathrm{M}$ on the erythrocyte interior was 
performed, followed by an injection of $100 \mu \mathrm{L}$ heparin. Fluorescence intensity from the injection was collected at the probe head, and processed by software written by Meissner and Ritter.

Instruments: ${ }^{1} \mathrm{H}-\mathrm{NMR}$ and ${ }^{13} \mathrm{C}-\mathrm{NMR}$ spectra were collected on a Bruker Avance III $500 \mathrm{MHz}$, or a Bruker Avance III $600 \mathrm{MHz}$. High Resolution mass spectra for characterization were performed by COSMIC at Old Dominion University on a Bruker Apex-Qe. Low Resolution mass spectra for reaction monitoring was performed on a Thermo LCQ Deca XP. Absorbance measurements were performed on a Cary-Win 100 Spectrophotometer, and near-IR fluorescence measurements were performed on a Horiba Fluorolog-3 using an InGaAs detector. Slit widths for both excitation and emission were set to $15 \mathrm{~nm}$. All centrifugations were performed on a TOMY MX150.

Erythrocyte Isolation and Washing Fresh whole blood pooled from male Sprague-Dawley rats, purchased from either Hemostat Laboratories or Biochemed Services was diluted approximately 1:1 in $165 \mathrm{mM}(1 \mathrm{x})$ PBS in a centrifuge tube and pelleted at 2,500 rpm for 8 minutes at $4{ }^{\circ} \mathrm{C}$. Supernatant was removed and pelleted cells were resuspended in PBS at approximately 5 - 10:1 dilution factor. This process was repeated twice. After the third wash, pelleted erythrocytes were then used directly in erythrocyte loading procedures. Following erythrocyte loading, similar washing steps were performed. Erythrocytes recovered from loading procedures were suspended in PBS, and centrifuged at 2,500 rpm at $4{ }^{\circ} \mathrm{C}$ for 8 minutes. The supernatant was removed and pelleted erythrocytes were resuspended in PBS and centrifuged again under the same conditions. This process was repeated a third time, and pelleted erythrocytes were used in experimental procedures. 


\section{A.2 Compound Characterization}

Synthesis of IR-823-N-Methylpiperazine (11). In a flame dried, $\mathrm{N}_{2}$ flushed flask with stirbar, IR-823 (45 mg, $0.042 \mathrm{mmol}$ ), and tetrakis(triphenylphosphine)palladium(0) (5 mg, $0.004 \mathrm{mmol}$ ) were dissolved in anhydrous DMF (6 mL). 1Methylpiperazine $(0.2 \mathrm{ml}, 1.8 \mathrm{mmol})$ was added via syringe, and the reaction was set to stir at $60{ }^{\circ} \mathrm{C}$ overnight. The solvent was removed under reduced pressure, and the crude material was redissolved in DI $\mathrm{H}_{2} \mathrm{O}$ and purified by $\mathrm{HPLC}$ with a gradient of $\mathrm{H}_{2} \mathrm{O}$ /Acetonitrile. Fractions were collected based on absorbance and condensed to afford a glossy blue/gold solid (21 mg, 42\%). ${ }^{1} \mathrm{H}-\mathrm{NMR}(500 \mathrm{MHz}, \mathrm{MeOD}): \delta=1.89$ $(\mathrm{t}, 2 \mathrm{H}, J=6.0 \mathrm{~Hz}), 1.99(\mathrm{~m}, 12 \mathrm{H}), 2.02(\mathrm{~s}, 12 \mathrm{H}), 2.54(\mathrm{~s}, 3 \mathrm{H}), 2.61(\mathrm{t}, 4 \mathrm{H}, J=6.5$ Hz), 2.68 (br, 4H), $2.92(\mathrm{t}, 4 \mathrm{H}, J=7 \mathrm{~Hz}), 2.99$ (br, 4H), 3.87 (br, 4H), 4.21 (t, 4H, $J=7 \mathrm{~Hz}), 6.11(\mathrm{~d}, 2 \mathrm{H}, J=14 \mathrm{~Hz}), 7.63(\mathrm{~d}, 2 \mathrm{H}, J=8.5 \mathrm{~Hz}), 7.92(\mathrm{~d}, 2 \mathrm{H}, J=$ $13.5 \mathrm{~Hz}), 7.99(\mathrm{dd}, 2 \mathrm{H}, J=9.0,1.5 \mathrm{~Hz}), 8.06(\mathrm{~d}, 2 \mathrm{H}, J=8.5 \mathrm{~Hz}), 8.28(\mathrm{~d}, 2 \mathrm{H}, J$ $=9.0 \mathrm{~Hz}), 8.40(\mathrm{~d}, 2 \mathrm{H}, J=1.5 \mathrm{~Hz}) \cdot{ }^{13} \mathrm{C}-\mathrm{NMR}(125 \mathrm{MHz}, \mathrm{MeOD}): \delta=23.0,23.6$, 26.0, 27.3, 28.8, 44.6, 44.7, 46.2, 51.3, 51.9, 55.0, 57.6, 98.1, 112.8, 123.6, 125.8, 126.8, 128.5, 130.0, 131.7, 132.6, 133.8, 141.8, 142.7, 143.0, 172.5. HRMS (ESI-) Calcd for [M - 2Na+]: 535.1460, found: 535.1458. 


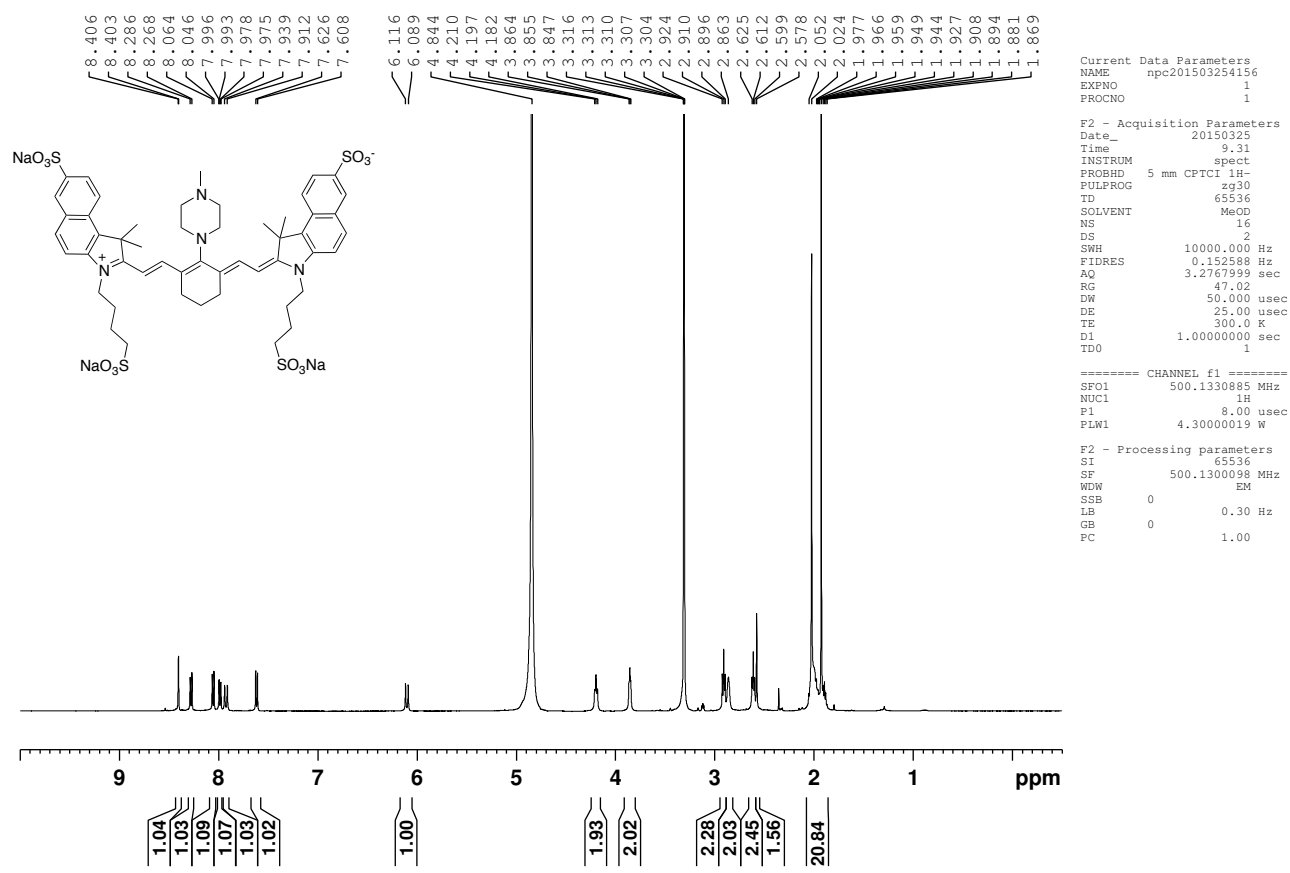

npc $201503 \quad 254156$
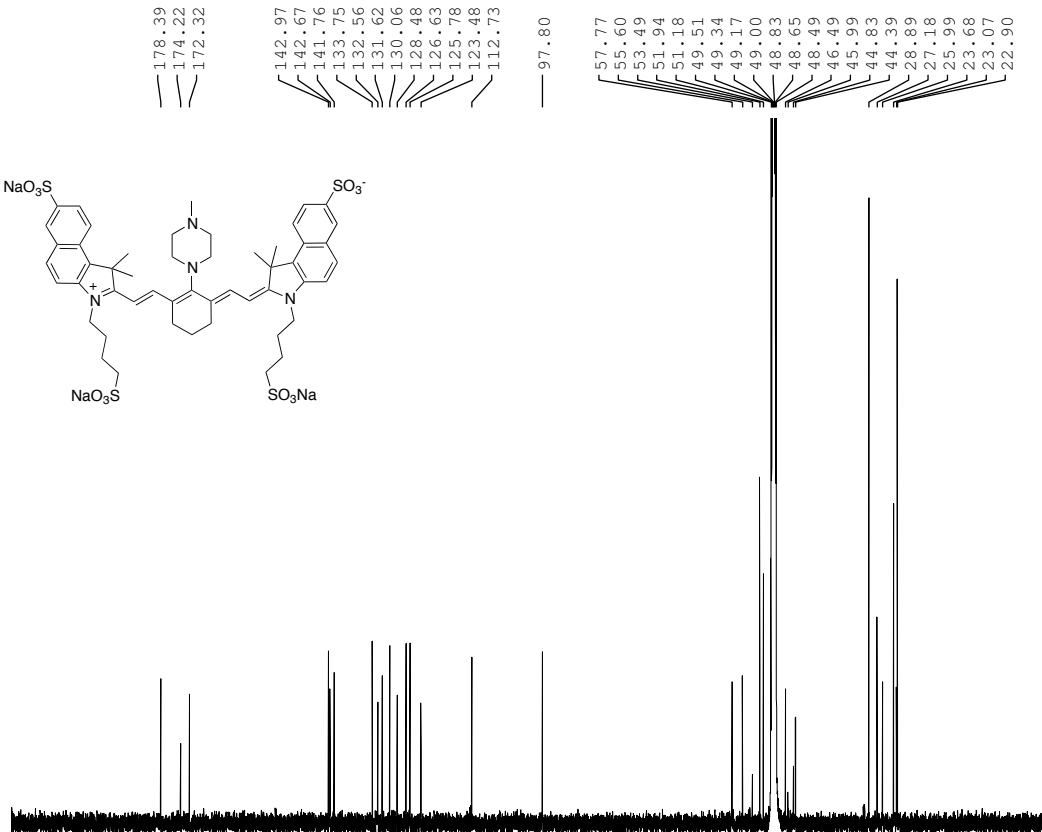

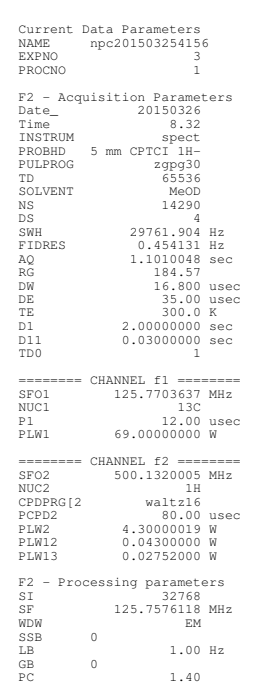


Synthesis of IR-823-N-Trimethylethylenediamine (12). In a flame dried, $\mathrm{N}_{2}$ flushed flask with stirbar, IR-823 (40 mg, $0.037 \mathrm{mmol}$ ), and tetrakis(triphenylphosphine)palladium(0) (6 mg, $0.005 \mathrm{mmol}$ ) were dissolved in anhydrous DMF. Trimethylethylenediamine $(0.05 \mathrm{ml}, 0.384 \mathrm{mmol})$ was added via syringe. Reaction was set to stir in oil bath at $70{ }^{\circ} \mathrm{C}$. After 30 minutes an aliquot of the reaction was removed and diluted in neutral PBS, and the absorbance was scanned, indicating consumption of starting material. The solvent was removed under reduced pressure, and the crude material was redissolved in DI $\mathrm{H}_{2} \mathrm{O}$ and purified by HPLC with a gradient of $\mathrm{H}_{2} \mathrm{O} /$ Acetonitrile. Fractions were collected based on absorbance and condensed to afford a matte blue solid (30 mg, 70\%). ${ }^{1} \mathrm{H}-\mathrm{NMR}(500 \mathrm{MHz}, \mathrm{MeOD}): \delta=1.96$ (m, $5 \mathrm{H}), 2.00(\mathrm{~s}, 12 \mathrm{H}), 2.05(\mathrm{~m}, 4 \mathrm{H}), 2.64(\mathrm{t}, 4 \mathrm{H}, J=6.0 \mathrm{~Hz}), 2.92(\mathrm{~m}, 10 \mathrm{H}), 3.35(\mathrm{~s}$, $6 \mathrm{H}), 3.46(\mathrm{~s}, 3 \mathrm{H}), 4.03(\mathrm{t}, 2 \mathrm{H}, J=7.5 \mathrm{~Hz}), 4.27(\mathrm{t}, 4 \mathrm{H}, J=3.0 \mathrm{~Hz}), 6.2(\mathrm{~d}, 2 \mathrm{H}$, $J=13.5 \mathrm{~Hz}), 7.64(\mathrm{~d}, 2 \mathrm{H}, J=8.5 \mathrm{~Hz}), 7.85(\mathrm{~d}, 2 \mathrm{H} J=13.5 \mathrm{~Hz}), 8.0(\mathrm{dd}, 2 \mathrm{H}, J$ $=9.0 \mathrm{~Hz}, J=1.5 \mathrm{~Hz}), 8.08(\mathrm{~d}, 2 \mathrm{H}, J=9.0 \mathrm{~Hz}), 8.28(\mathrm{~d}, 2 \mathrm{H}, J=9.0 \mathrm{~Hz}), 8.42$ $(\mathrm{d}, 2 \mathrm{H}, J=1.5 \mathrm{~Hz}) .{ }^{13} \mathrm{C}-\mathrm{NMR}(125 \mathrm{MHz}, \mathrm{MeOD}): \delta=22.9,23.0,23.7,23.8,27.1$, 28.82, 29.4, 44.2, 45.7, 50.8, 52.0, 58.0, 96.3, 112.5, 123.4, 125.7, 128.5, 130.2, 131.3, 132.5, 133.1, 141.4, 141.6, 143.2, 171.2, 176.8. HRMS (ESI-) Calcd for [M - 2Na+]: 527.6405, found: 527.6403 . 


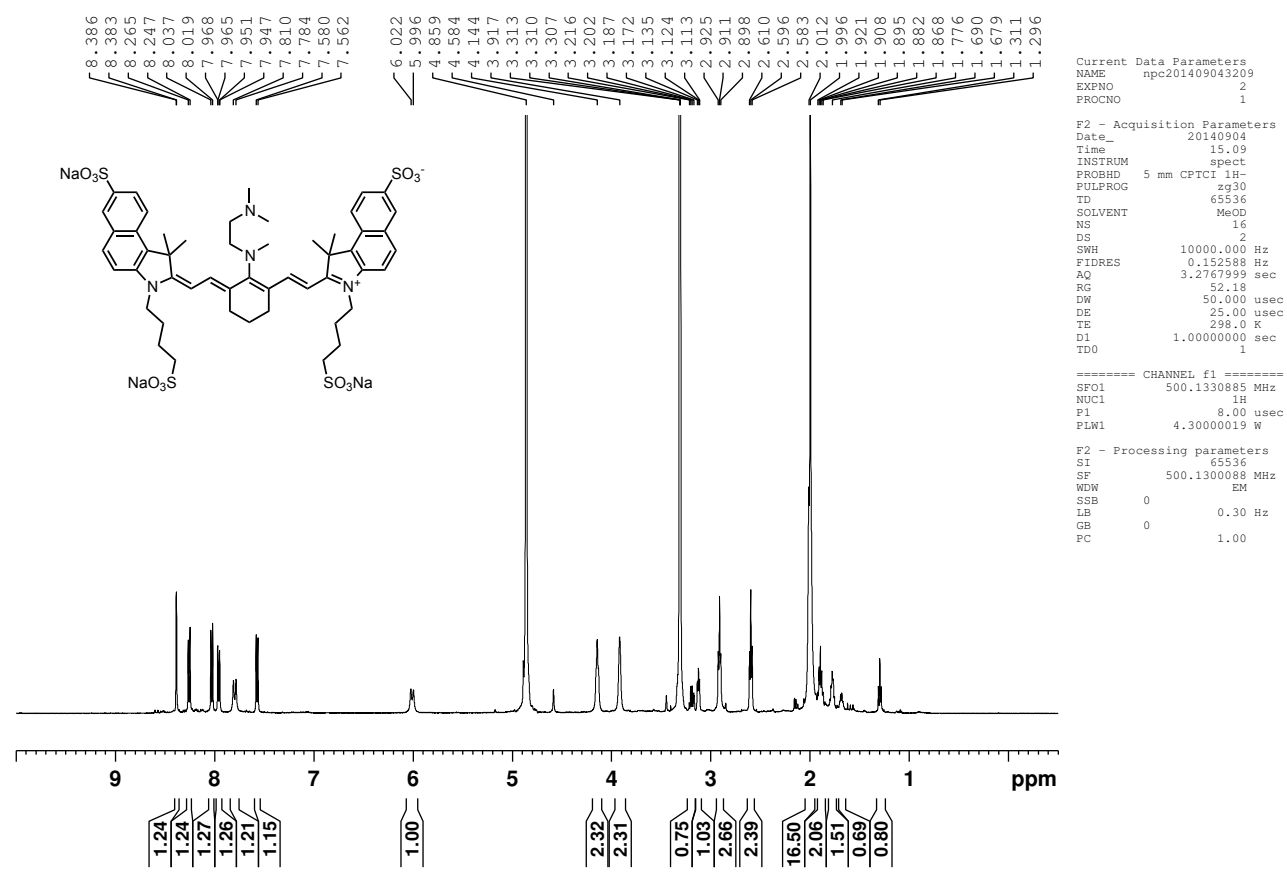

npc $20140904 \quad 3209$

Compound $12 \mathrm{C}-\mathrm{NMR}$ 125MHz MeOD
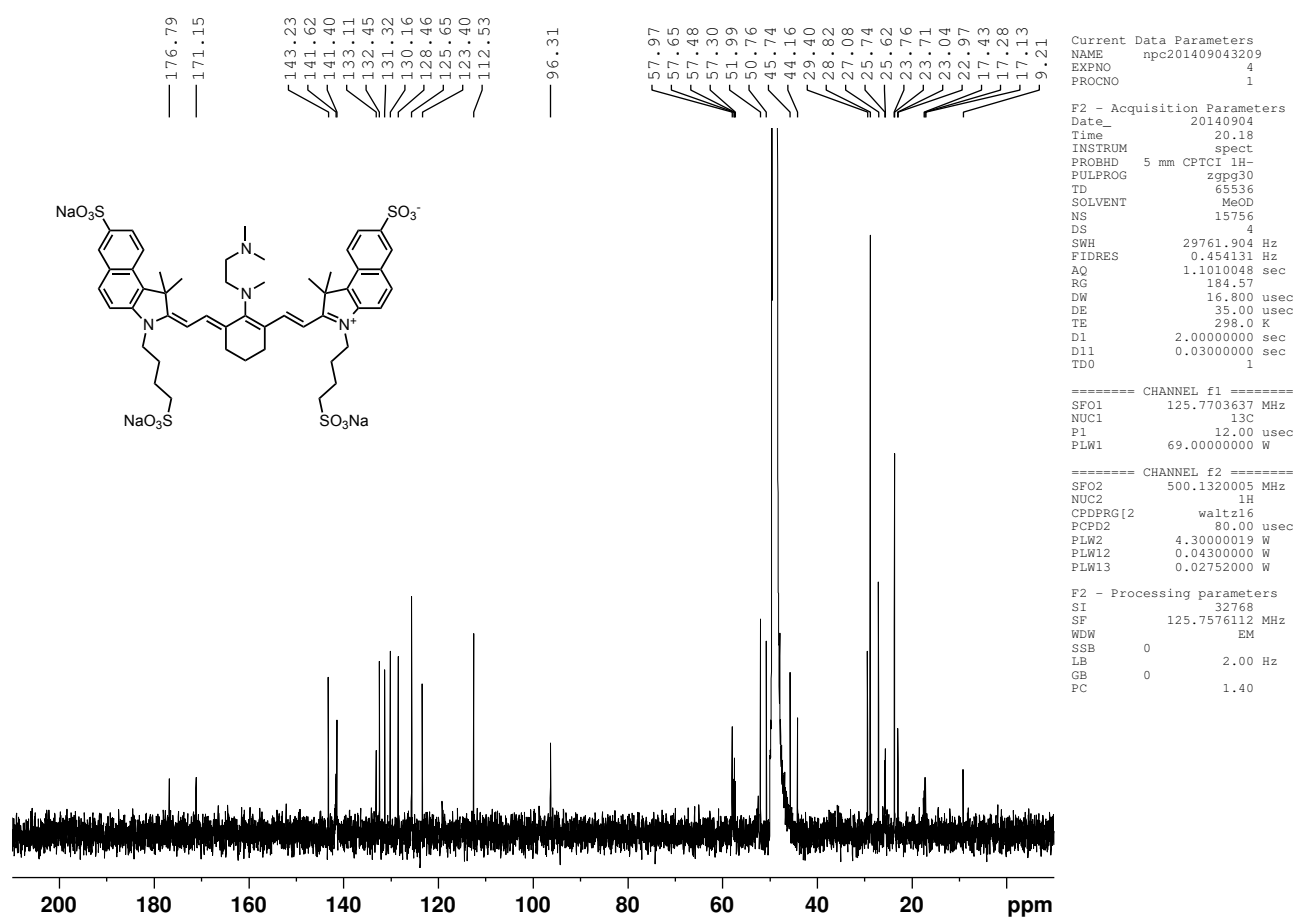
Synthesis of IR-823-Piperidine (13). In a flame dried, $\mathrm{N}_{2}$ flushed flask with stirbar, IR-823 (32 mg, $0.03 \mathrm{mmol}$ ), and tetrakis(triphenylphosphine)palladium(0) (5 $\mathrm{mg}, 0.004 \mathrm{mmol})$ were dissolved in anhydrous DMF (6 ml). Piperidine $(0.1 \mathrm{ml}, 1.01$ mmol) was added via syringe, and the reaction was set to stir at $75^{\circ} \mathrm{C}$. After stirring for 3 hours an aliquot of the reaction was removed and diluted in neutral PBS, and the absorbance was scanned, indicating complete consumption of starting material. The solvent was removed under reduced pressure, and the crude material redissolved in DI $\mathrm{H}_{2} \mathrm{O}$ and purified by HPLC with a gradient of $\mathrm{H}_{2} \mathrm{O} /$ Acetonitrile. Fractions were collected based on absorbance and condensed to afford a matte blue solid (19.6 mg, 59\%). ${ }^{1} \mathrm{H}-\mathrm{NMR}(500 \mathrm{MHz}, \mathrm{MeOD}): \delta=1.30$ (t, 2H, $\left.J=7.0 \mathrm{~Hz}\right), 1.78(\mathrm{br}, 3 \mathrm{H})$, $1.90(\mathrm{tt}, 4 \mathrm{H}, J=6.5 \mathrm{~Hz}, J=6.5 \mathrm{~Hz}), 2.0(\mathrm{br}, 32 \mathrm{H}), 2.60(\mathrm{t}, 4 \mathrm{H}, J=7.0 \mathrm{~Hz}), 2.92$ $(\mathrm{t}, 4 \mathrm{H}, J=7.0 \mathrm{~Hz}), 3.12(\mathrm{t}, 2 \mathrm{H}, J=5.5 \mathrm{~Hz}), 3.92(\mathrm{br}, 4 \mathrm{H}), 4.14(\mathrm{br}, 4 \mathrm{H}), 6.02$ $(\mathrm{d}, 2 \mathrm{H}, J=13 \mathrm{~Hz}), 7.80(\mathrm{~d}, 2 \mathrm{H}, J=17.0 \mathrm{~Hz}), 7.96(\mathrm{dd}, 2 \mathrm{H}, J=8.5 \mathrm{~Hz}, J=1.5$ $\mathrm{Hz}), 8.03(\mathrm{~d}, 2 \mathrm{H}, J=9.0 \mathrm{~Hz}), 8.26(\mathrm{~d}, 2 \mathrm{H}, J=9.0 \mathrm{~Hz}), 8.38(\mathrm{~d}, 2 \mathrm{H}, J=1.5 \mathrm{~Hz})$. ${ }^{13} \mathrm{C}-\mathrm{NMR}(125 \mathrm{MHz}, \mathrm{MeOD}): \delta=23.0,23.5,28.9,44.4,44.6,45.8,51.6,51.9,56.8$, 99.5, 113.0, 123.5, 125.9, 127.4, 128.5, 130.0, 131.9, 132.7, 134.2, 142.1, 142. 8, 143.4, 173.6. HRMS (ESI-) Calcd for [M - 2Na+]: 536.1538, found: 536.1537. 


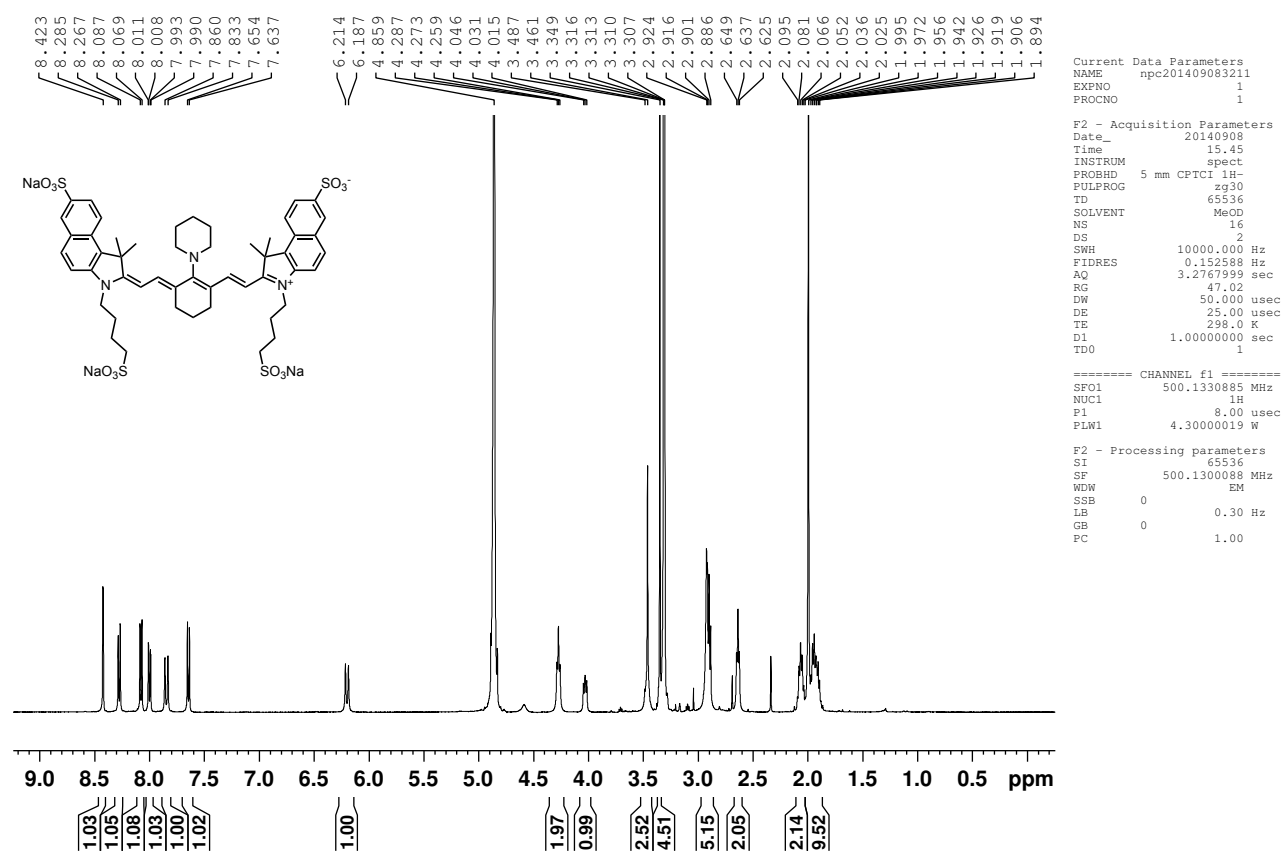

npc $201409083211 \quad$ Compound 13 C-NMR 125MHz MeOD

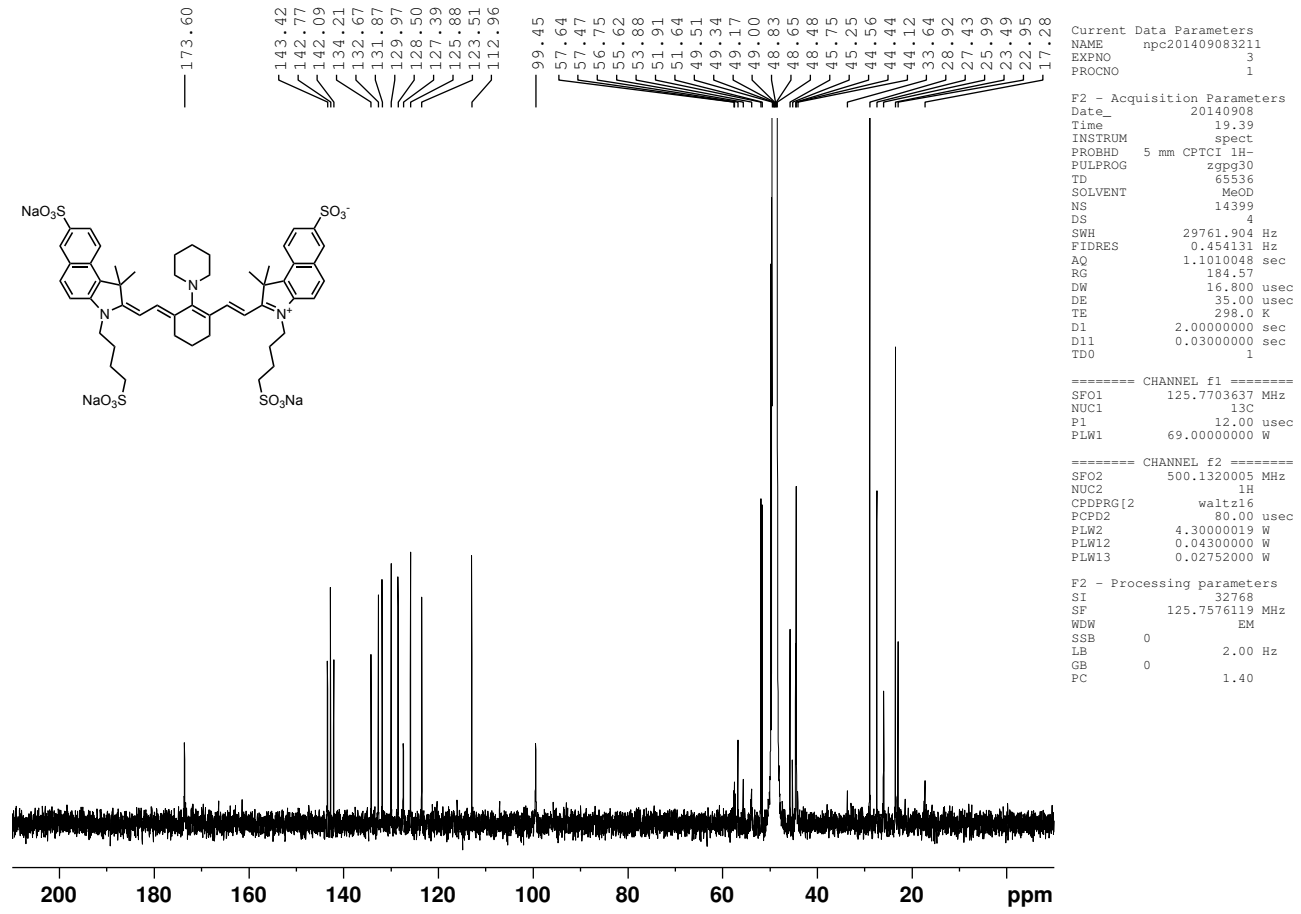


Synthesis of IR-823-4-Methylpiperazynl-Phenyl (14). In a 2 neck flask with condenser, IR-823 (30 mg, $0.028 \mathrm{mmol}$ ), tetrakis(triphenylphosphine)palladium(0) (4 mg, $0.003 \mathrm{mmol})$, tribasic potassium phosphate $(30 \mathrm{mg}, 0.141 \mathrm{mmol})$ and (4-(4methylpiperazin-1-yl)phenyl)boronic acid (32 $\mathrm{mg}, 0.145 \mathrm{mmol}$ ) were dissolved in DI $\mathrm{H}_{2} \mathrm{O}$ and the reaction was set to reflux. After 40 minutes an aliquot of the reaction was removed and diluted in neutral PBS, and it's absorbance was scanned, indicating complete consumption of starting material. Reaction was condensed under reduced pressure and the crude material redissolved in DI $\mathrm{H}_{2} \mathrm{O}$ and purified by HPLC with a gradient of $\mathrm{H}_{2} \mathrm{O} /$ Acetonitrile. Fractions were collected based on absorbance and condensed to afford a green solid (16.6 mg, 49\%). ${ }^{1} \mathrm{H}-\mathrm{NMR}$ (500 MHz, MeOD): $\delta=$ $1.56(\mathrm{~s}, 12 \mathrm{H}), 1.94(\mathrm{br}, 8 \mathrm{H}), 2.08(\mathrm{t}, 2 \mathrm{H}, J=4.0 \mathrm{~Hz}), 2.78(\mathrm{t}, 4 \mathrm{H}, J=5.5 \mathrm{~Hz}), 2.90$ (t, $4 \mathrm{H}, J=7.0 \mathrm{~Hz}), 2.97(\mathrm{~s}, 3 \mathrm{H}), 3.47(\mathrm{br}, 4 \mathrm{H}), 3.65(\mathrm{br}, 4 \mathrm{H}), 4.21$ (br, 4H), $6.28(\mathrm{~d}$, $2 \mathrm{H} J=14.0 \mathrm{~Hz}), 7.23(\mathrm{~d}, 2 \mathrm{H} J=8.5 \mathrm{~Hz}), 7.35(\mathrm{~d}, 2 \mathrm{H}, J=8.5 \mathrm{~Hz}), 7.46(\mathrm{~d}, 2 \mathrm{H}, J$ $=14.0 \mathrm{~Hz}), 7.63(\mathrm{~d}, 2 \mathrm{H}, J=9.0 \mathrm{~Hz}), 7.96(\mathrm{dd}, 2 \mathrm{H}, J=9.0 \mathrm{~Hz}, J=1.5 \mathrm{~Hz}), 8.057$ $(\mathrm{m}, 4 \mathrm{H}), 8.04(\mathrm{~d}, 2 \mathrm{H}, 1.0 \mathrm{~Hz}) .{ }^{13} \mathrm{C}-\mathrm{NMR}(125 \mathrm{MHz}, 1: 1 \mathrm{MeOD}: \mathrm{H} 2 \mathrm{O}): \delta 22.3,23.2$, $25.6,27.19,27.6,44.0,44.8,51.4,51.7,54.7,100.7,113.0,117.9,123.7,125.4,128.4$, 129.6, 131.7, 131.9, 132.4, 132.8, 133.7, 134.0, 141.0, 142.4, 148.6, 151.2, 163.6, 170.9, 174.2. HRMS (ESI+) Calcd for [M $+1 \mathrm{Na}+, 1 \mathrm{H}+]$ : 608.1491, found: 608.1491. 


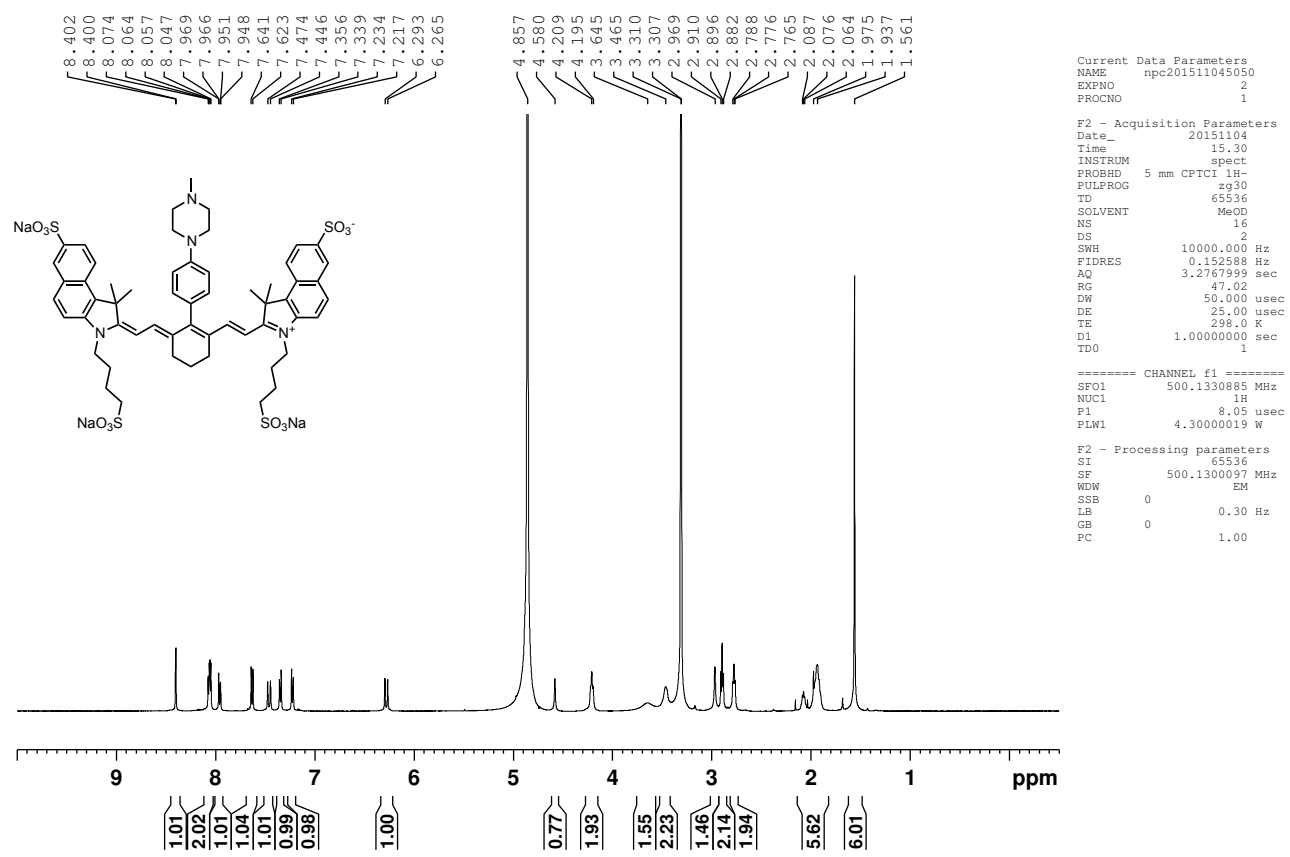

npc $20151106 \quad 5050$

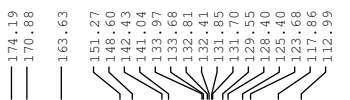

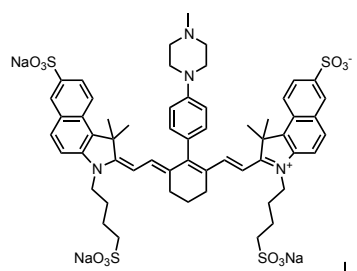

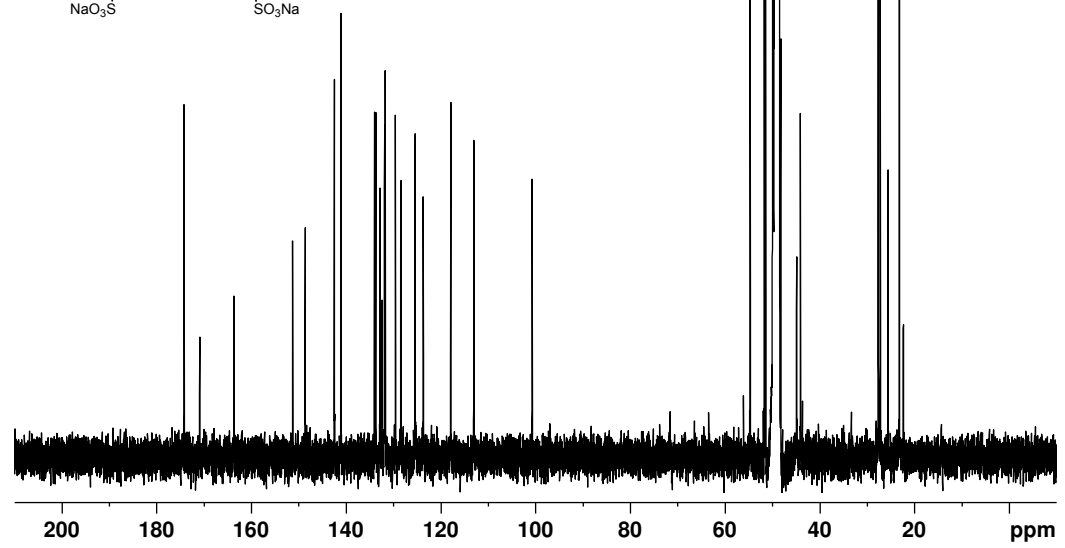

Compound 14 C-NMR 125MHz 1:1 MeOD: $\mathrm{D}_{2} \mathrm{O}$

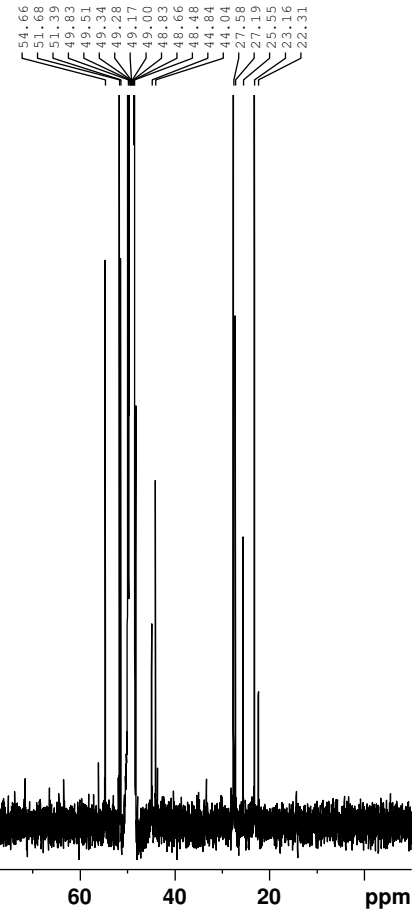

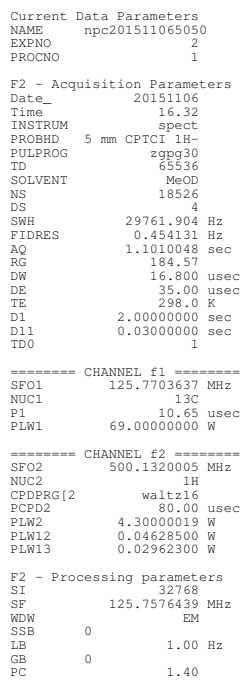


Synthesis of IR-823-Piperazine (44) In a flame dried $\mathrm{N}_{2}$ flushed flask with stirbar IR-823 (35 mg, $0.033 \mathrm{mmol}$ ), tetrakis(triphenylphosphine)palladium(0) (6 mg, $0.005 \mathrm{mmol}$ ), and piperazine (20 mg, $0.232 \mathrm{mmol}$ ) were dissolved in dry DMF ( $5 \mathrm{~mL}$ ) and set to stir in an oil bath at $65^{\circ} \mathrm{C}$. After 7 hours the reaction was removed from heat and condensed under reduced pressure. The crude material was redissolved in DI $\mathrm{H}_{2} \mathrm{O}$ and purified by HPLC with a $\mathrm{H}_{2} \mathrm{O} /$ Acetonitrile. Fractions were collected based on absorbance to afford a matte blue solid (18.7 mg, 51\%). ${ }^{1} \mathrm{H}-\mathrm{NMR}$ (500 $\mathrm{MHz}, \mathrm{MeOD}): \delta=2.0(\mathrm{~s}, 12 \mathrm{H}), 2.03(\mathrm{~m}, 10 \mathrm{H}), 2.62(\mathrm{t}, 4 \mathrm{H}, J=6.5 \mathrm{~Hz}), 2.92(\mathrm{t}, 4 \mathrm{H}$, $J=7.5 \mathrm{~Hz}), 3.87(\mathrm{br}, 4 \mathrm{H}), 4.22(\mathrm{t}, 4 \mathrm{H}, J=7 \mathrm{~Hz}), 6.14(\mathrm{~d}, 2 \mathrm{H}, J=13.5 \mathrm{~Hz}), 7.63$ $(\mathrm{d}, 2 \mathrm{H}, J=9 \mathrm{~Hz}), 7.92(\mathrm{~d}, 2 \mathrm{H}, J=13.5 \mathrm{~Hz}), 8.00(\mathrm{dd}, 2 \mathrm{H}, J=9 \mathrm{~Hz}, 1.5 \mathrm{~Hz}), 8.07$ (d, $2 \mathrm{H}, J=9 \mathrm{~Hz}), 8.25$ (d, 2H, $J=9 \mathrm{~Hz}), 8.42(\mathrm{~d} 2 \mathrm{H}, 1.5 \mathrm{~Hz})$. HRMS (ESI-) Calcd for [M - 2Na+]: 528.1382, found: 528.1381. 


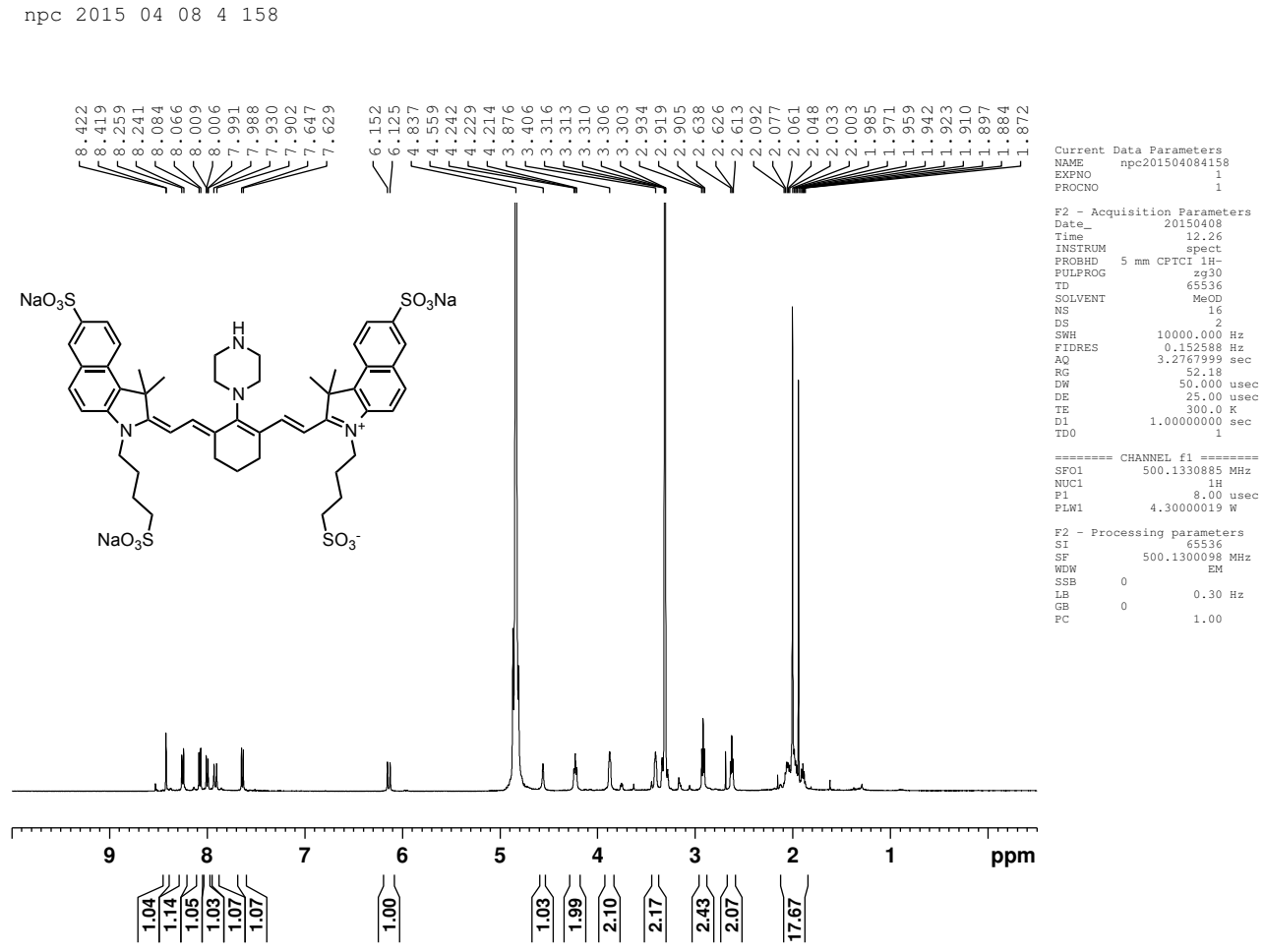


Synthesis of IR-823-Piperazynlbenzyl boronic acid (45) In a flask with stirbar, crude $44(18.7 \mathrm{mg}, 0.016 \mathrm{mmol})$ was dissolved in 1:3:3 $\mathrm{H}_{2} \mathrm{O}$, acetonitrile and methanol, (7 ml). (2-(bromomethyl)phenyl)boronic acid (40 mg, $0.186 \mathrm{mmol})$ and tribasic potassium phosphate $(40 \mathrm{mg}, 0.188 \mathrm{mmol}$ ) were added, and the reaction was set to stir at $85^{\circ} \mathrm{C}$ overnight under $\mathrm{N}_{2}$ atmosphere. The reaction was condensed under reduced pressure and the crude material was redissolved in $\mathrm{H}_{2} \mathrm{O}$ and subjected to HPLC purification on a gradient of $\mathrm{H}_{2} \mathrm{O}$ /Acetonitrile. Fractions were collected based on absorbance to afford a matte green solid (11.8 mg, $29 \%$ over 2 steps). ${ }^{1} \mathrm{H}$ $\operatorname{NMR}(600 \mathrm{MHz}, \mathrm{MeOD}): \delta=1.98(\mathrm{~m}, 10 \mathrm{H}), 2.05(\mathrm{~s}, 12 \mathrm{H}), 2.62(\mathrm{t}, 4 \mathrm{H}, J=6.6 \mathrm{~Hz})$, $2.91(\mathrm{t}, 4 \mathrm{H}, J=7.2), 3.03(\mathrm{br}, 4 \mathrm{H}), 3.91(\mathrm{br}, 4 \mathrm{H}), 4.04(\mathrm{~s}, 2 \mathrm{H}), 4.21(\mathrm{t}, 4 \mathrm{H}, J=7.5)$, $6.12(\mathrm{~d}, 2 \mathrm{H}, J=13.2), 7.34(\mathrm{~m}, 3 \mathrm{H}), 7.62(\mathrm{br}, 1 \mathrm{H}), 7.63(\mathrm{~d}, 2 \mathrm{H}, J=9.0 \mathrm{~Hz}), 7.94(\mathrm{~d}$, $2 \mathrm{H}, J=13.8 \mathrm{~Hz}), 8.01(\mathrm{dd}, 2 \mathrm{H}, J=9.0 \mathrm{~Hz}, J=1.8 \mathrm{~Hz}), 8.07(\mathrm{~d}, 2 \mathrm{H}, J=9.6 \mathrm{~Hz})$, $8.31(\mathrm{~d}, 2 \mathrm{H}, J=9.0 \mathrm{~Hz}), 8.41(\mathrm{~d}, 2 \mathrm{H}, J=1.8 \mathrm{~Hz}){ }^{13} \mathrm{C}-\mathrm{NMR}(150 \mathrm{MHz}, \mathrm{MeOD}): \delta=$ 23.0, 23.2, 23.5, 23.7, 26.0, 27.2, 29.0, 44.33, 51.2, 51.9, 54.4, 55.1, 97.9, 112.8123 .5 , 125.8, 126.6, 128.2, 128.5, 130.1, 131.6, 132.6, 133.7, 141.7, 142.0, 142.5, 143.0, 172.2, 173.8, 178.9. HRMS (ESI-) Calcd for [M - 2Na+]: 595.1652, found: 595.1654. 
Compound $45 \mathrm{H}-\mathrm{NMR} 600 \mathrm{MHz}$ MeOD

npc $2015 \quad 04 \quad 28 \quad 4 \quad 178$

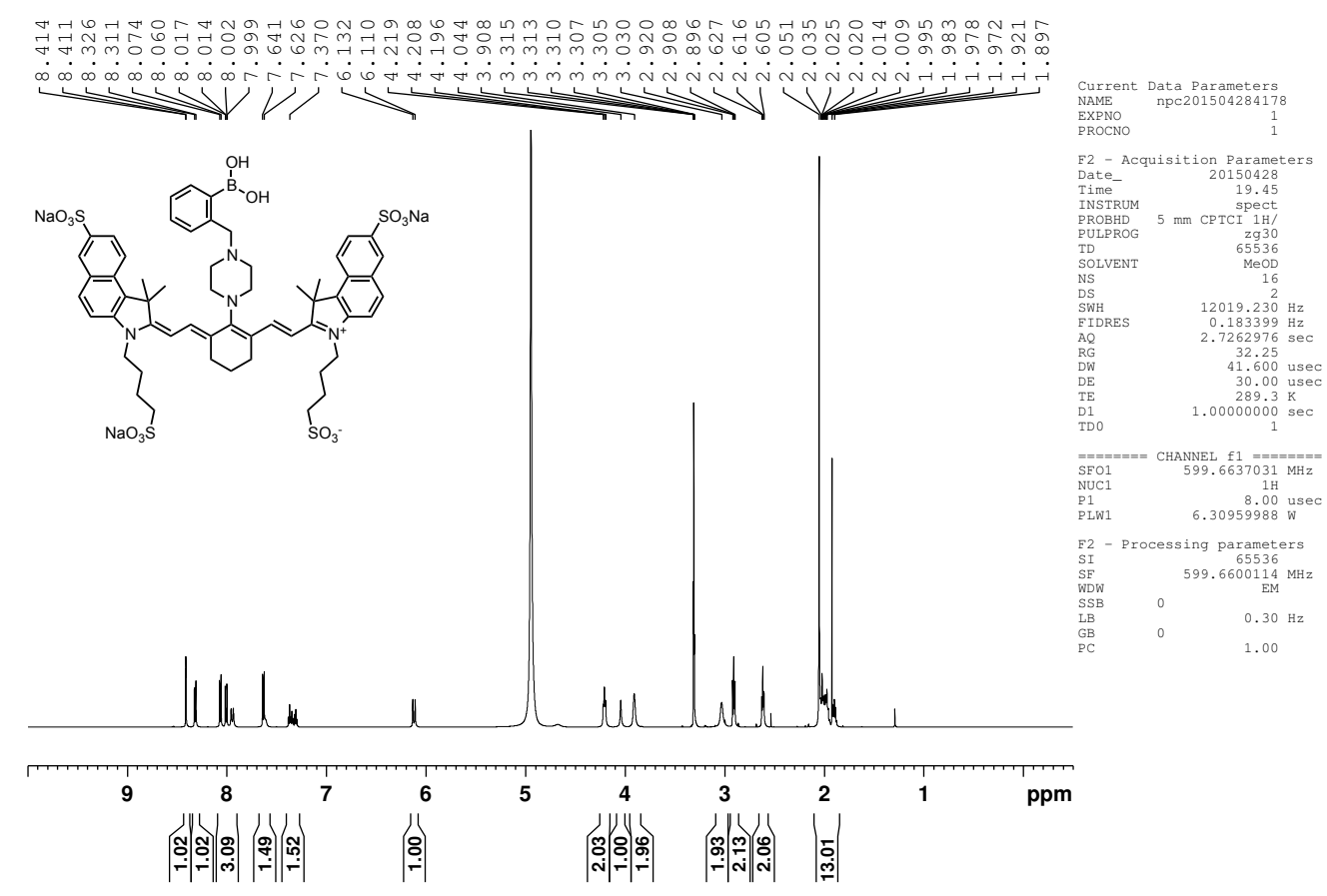

npc $2015 \quad 04 \quad 28 \quad 4 \quad 178$

Compound 45 C-NMR 150 MHz MeOD

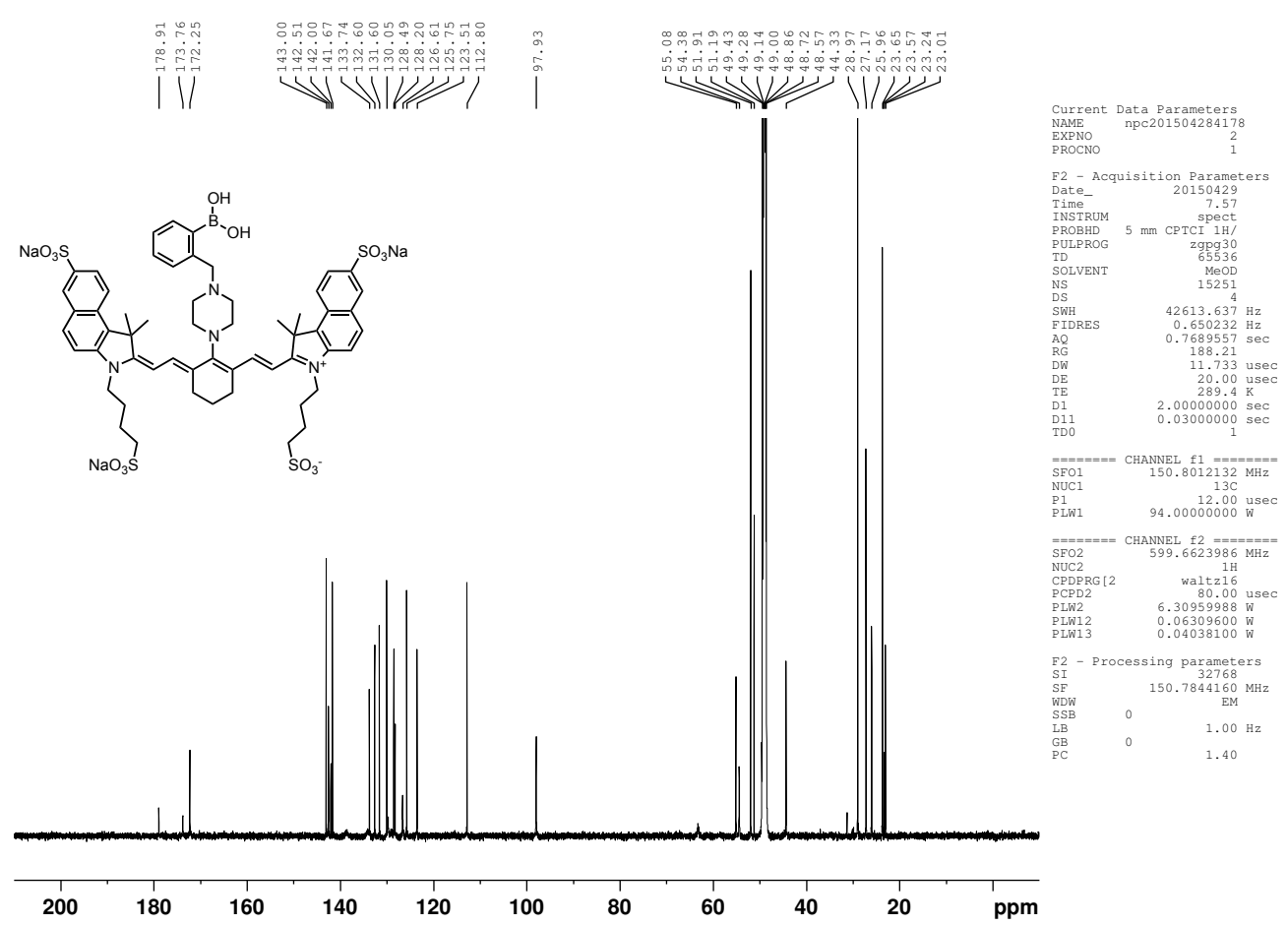




\section{Synthesis of IR-823-Piperazynl-fluoro-benzylphenyl boronic acid (46)}

In a flask with stirbar, crude 44 directly from the HPLC was dissolved in 1:3:3 $\mathrm{H}_{2} \mathrm{O}$, acetonitrile and methanol $(7 \mathrm{ml})$. (2-(bromomethyl)-5-fluorophenyl)boronic acid (25 mg, $0.107 \mathrm{mmol}$ ), and tribasic potassium phosphate (40 mg, $0.188 \mathrm{mmol}$ ) were added. The reaction was set to stir at room temperature overnight under $\mathrm{N}_{2}$ atmosphere. The reaction was condensed under reduced pressure and the crude material was redissolved in DI $\mathrm{H}_{2} \mathrm{O}$ and subjected to HPLC purification. Fractions were collected based on absorbance and condensed to afford a matte green solid ( $7.1 \mathrm{mg}, 22 \%$ over 2 steps). ${ }^{1} \mathrm{H}-\mathrm{NMR}(600 \mathrm{MHz}, \mathrm{MeOD}): \delta=1.89(\mathrm{~m}, 2 \mathrm{H}), 1.99(\mathrm{~m}, 20 \mathrm{H}), 2.61(\mathrm{t}, 4 \mathrm{H}, J=6.6$ $\mathrm{Hz}), 2.91(\mathrm{t}, 4 \mathrm{H}, J=7.2), 2.96(\mathrm{br}, 1 \mathrm{H}), 3.03(\mathrm{br}, 3 \mathrm{H}), 3.83(\mathrm{t}, 1 \mathrm{H}, J=4.2), 3.93$ (br, 3H), $3.99(\mathrm{~s}, .5 \mathrm{H}) 4.02(\mathrm{~s}, 1.5 \mathrm{H}) 4.2(\mathrm{t}, 4 \mathrm{H}, J=6.9), 6.11(\mathrm{~d}, 2 \mathrm{H}, J=13.2 \mathrm{~Hz})$, $6.91(\mathrm{~m}, .25 \mathrm{H}), 6.98(\mathrm{~m}, .25 \mathrm{H}), 7.04(\mathrm{td}, .75 \mathrm{H}, J=8.4 \mathrm{~Hz}, J=2.0 \mathrm{~Hz}), 7.10(\mathrm{dd}$, $.25 \mathrm{H}, J=9.0 \mathrm{~Hz}, J=3.0 \mathrm{~Hz}), 7.15(\mathrm{dd}, .75 \mathrm{H}, J=10.2 \mathrm{~Hz}, J=1.8 \mathrm{~Hz}), 7.54(\mathrm{br}$, $.75 \mathrm{H}), 7.62(\mathrm{~m}, 2 \mathrm{H}), 7.94(\mathrm{~m}, 2 \mathrm{H}), 8.02(\mathrm{~m}, 2 \mathrm{H}), 8.03(\mathrm{~d}, 2 \mathrm{H}, J=9.0), 8.30(\mathrm{~d}, 2 \mathrm{H}$, $J=9.0 \mathrm{~Hz}), 8.14(\mathrm{~d}, 2 \mathrm{H}, J=1.8)$. 


\section{Compound $46 \mathrm{H}-\mathrm{NMR} 600 \mathrm{MHz} \mathrm{MeOD}$}

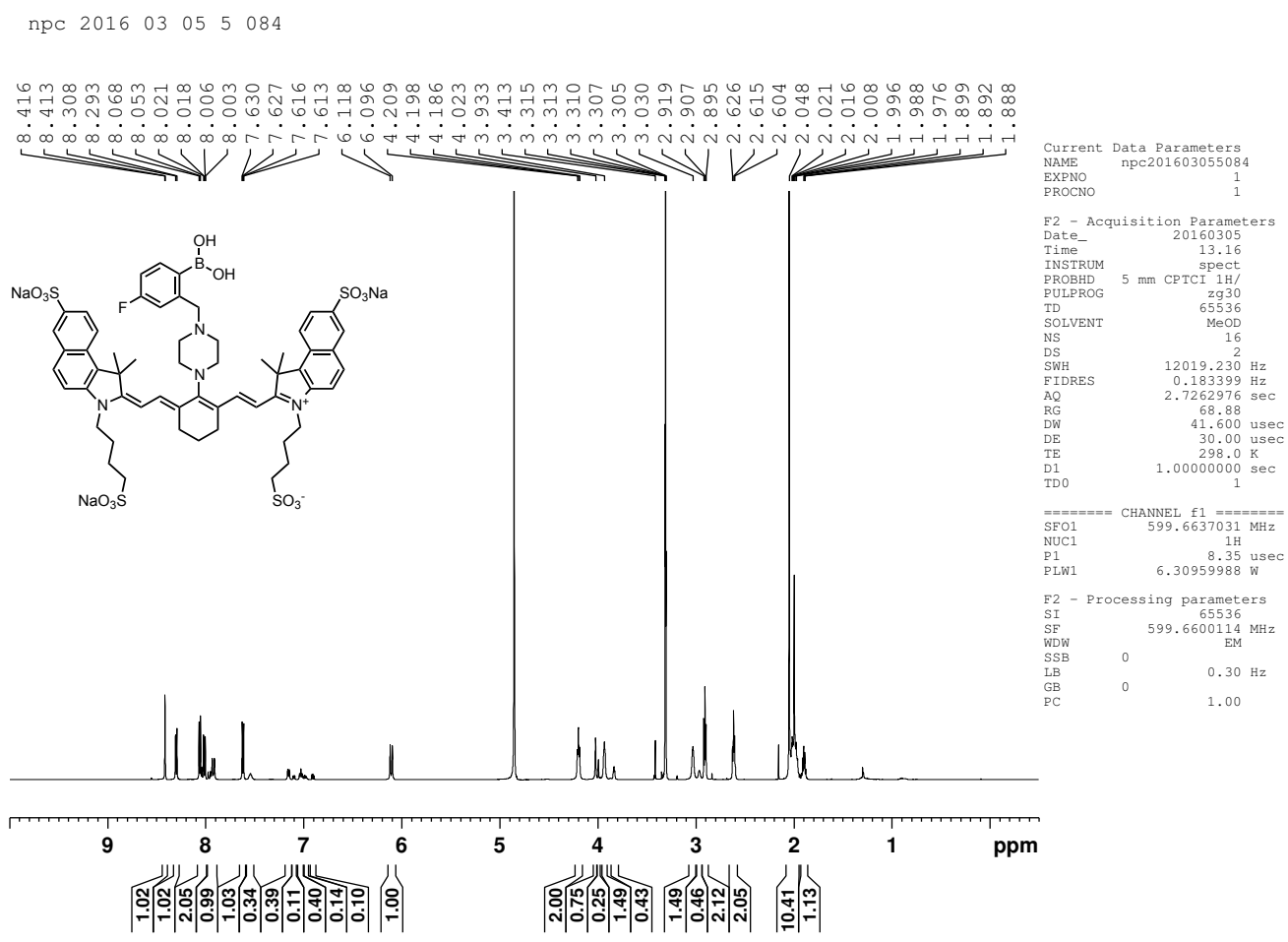


Synthesis of IR-823-Phenylethylene (18) In a flame dried, $\mathrm{N}_{2}$ flushed flask with stirbar, IR-823 (40 mg, $0.037 \mathrm{mmol}$ ), tetrakis(triphenylphosphine)palladium(0) (6 mg, $0.005 \mathrm{mmol}$ ), and copper iodide ( $8 \mathrm{mg}, 0.04 \mathrm{mmol}$ ) were dissolved in anhydrous DMF. Triethylamine $(0.05 \mathrm{ml}, 0.384 \mathrm{mmol})$ and phenylacetylene $(0.2 \mathrm{ml}, 1.8 \mathrm{mmol})$ were added via syringe. The reaction was set to stir in oil bath at $70{ }^{\circ} \mathrm{C}$. After 2 hours an absorbance check of reaction aliquot indicated consumption of starting material. The solvent was removed under reduced pressure, and the crude material was redissolved in $\mathrm{H}_{2} \mathrm{O}$ and purified by HPLC with a gradient of $\mathrm{H}_{2} \mathrm{O}$ /Acetonitrile. Fractions were collected based on absorbance to afford a green gold solid as the 2 $\mathrm{Na}^{+} 1 \mathrm{TEAH}^{+}$species $(7 \mathrm{mg}, 16 \%) .{ }^{1} \mathrm{H}-\mathrm{NMR}(500 \mathrm{MHz}, \mathrm{MeOD}): \delta=2.0(\mathrm{~m}, 12 \mathrm{H})$, $2.06(\mathrm{~s}, 12 \mathrm{H}), 2.78(\mathrm{t}, 4 \mathrm{H}, J=6 \mathrm{~Hz}), 2.93(\mathrm{t}, 4 \mathrm{H}, J=7 \mathrm{~Hz}), 4.33(\mathrm{t}, 4 \mathrm{H}, J=7 \mathrm{~Hz})$ $6.44(\mathrm{~d}, 2 \mathrm{H}, J=14 \mathrm{~Hz}), 7.64(\mathrm{tt}, 1 \mathrm{H}, J=7.5 \mathrm{~Hz}, J=1.5 \mathrm{~Hz}), 7.68(\mathrm{ddd}, 2 \mathrm{H}, J=$ $8.5 \mathrm{~Hz}, J=8.5 \mathrm{~Hz}, J=1.5 \mathrm{~Hz}), 7.72(\mathrm{~d}, 2 \mathrm{H}, J=9.0 \mathrm{~Hz}), 7.84(\mathrm{dd}, 2 \mathrm{H}, J=8.5$ $\mathrm{Hz}, J=1.5 \mathrm{~Hz}), 8.02(\mathrm{dd}, 2 \mathrm{H}, J=9.0 \mathrm{~Hz}, J=2 \mathrm{~Hz}), 8.11(\mathrm{~d}, 2 \mathrm{H} J=9 \mathrm{~Hz}), 8.26$ $(\mathrm{d}, 2 \mathrm{H} J=8.5 \mathrm{~Hz}), 8.44(\mathrm{~d}, 2 \mathrm{H}, J=2 \mathrm{~Hz}), 8.70(\mathrm{~d}, 2 \mathrm{H}, J=14 \mathrm{~Hz}) \cdot{ }^{13} \mathrm{C}-\mathrm{NMR}(125$ MHz, MeOD): $\delta=22.02,23.7,25.6,27.5,28.2,45.1,51.8,52.3,86.1,102.1,104.3$, 113.7, 123.3, 123.8, 126.1, 128.5, 129.9, 130.4, 131.0, 132.3, 132.9, 134.7, 135.0, 142.4, $142.5,146.3,174.6$. 


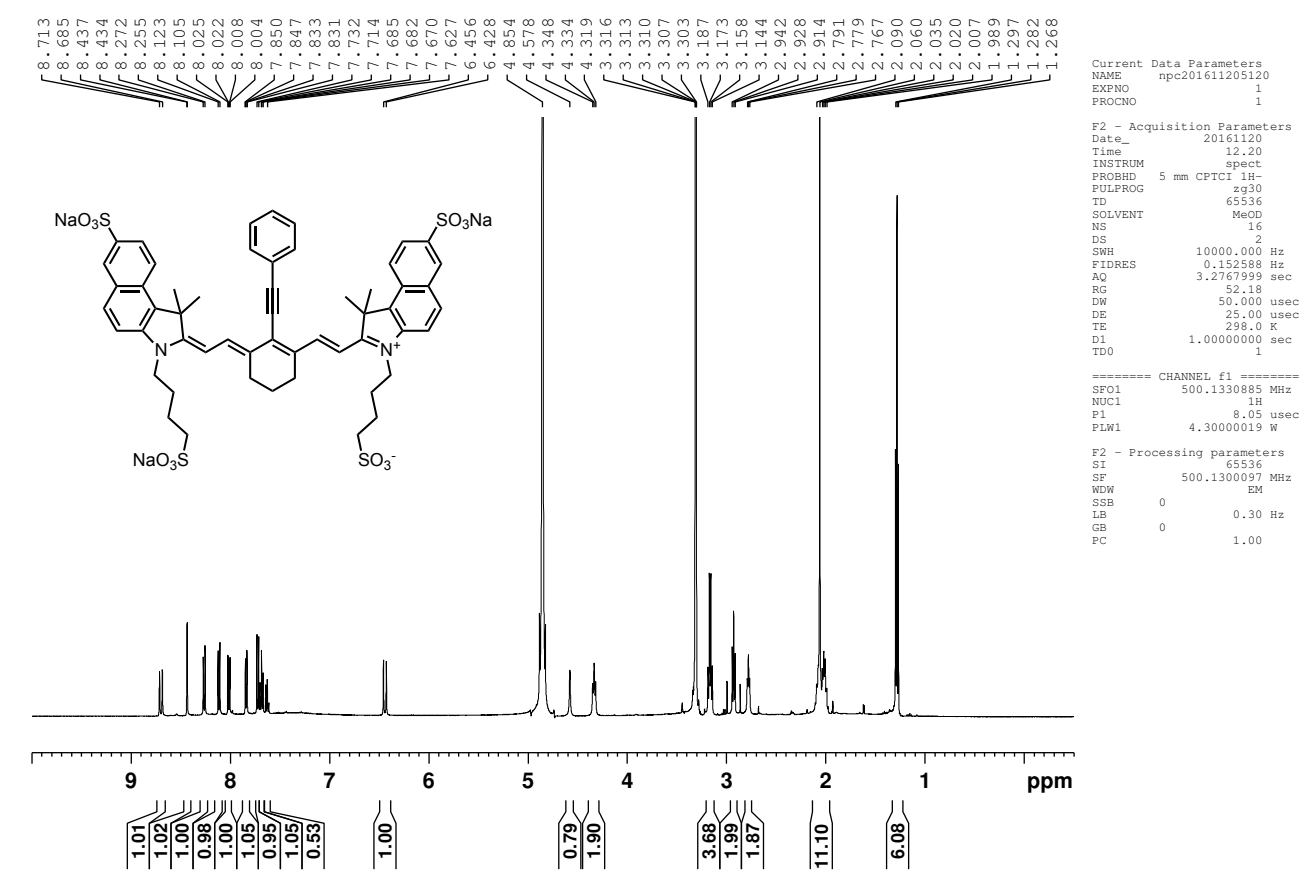

Compound 18 C-NMR 150 MHz MeOD

npc $20161120 \quad 5120$

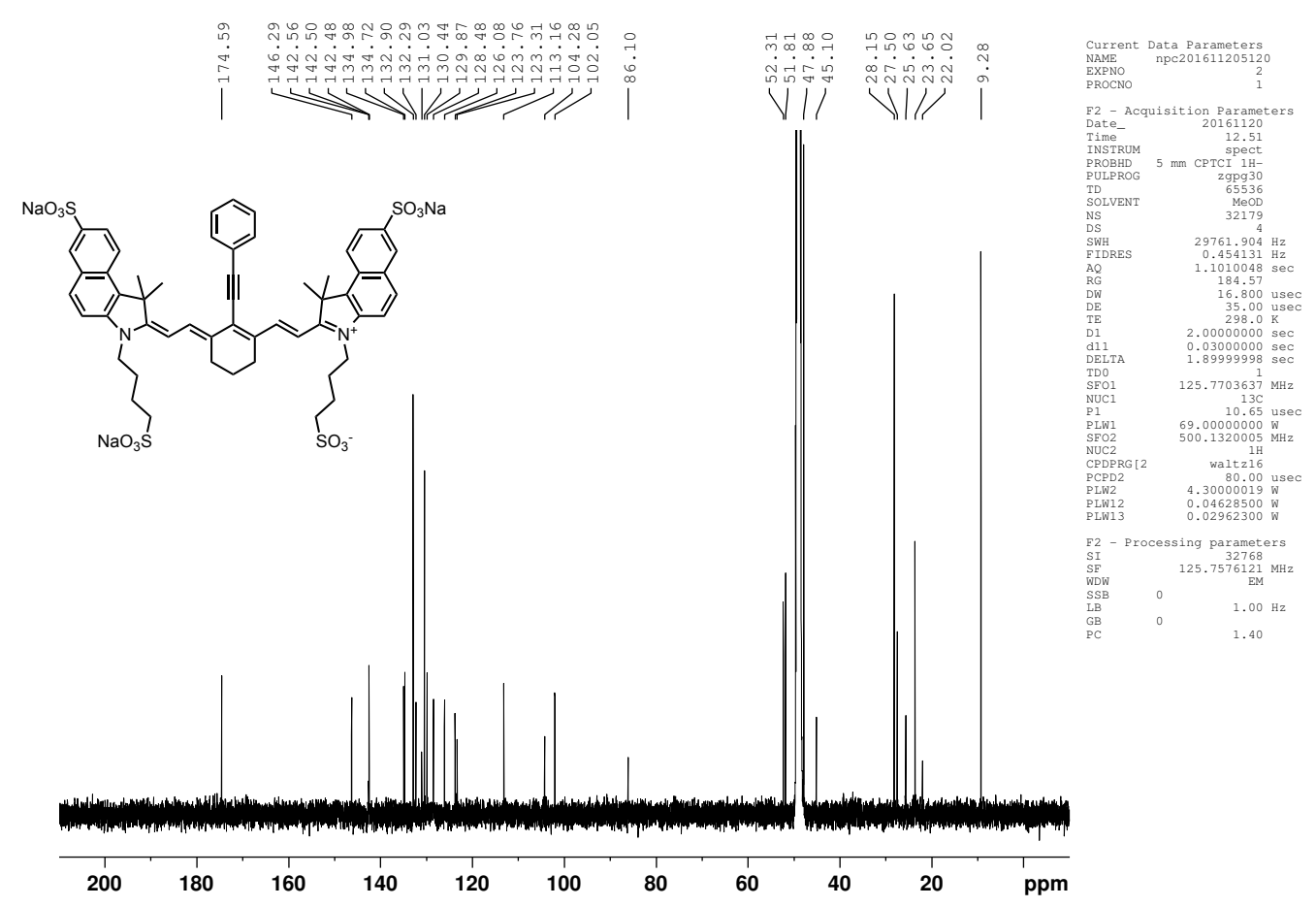




\section{Bibliography}

[1] Joseph R. Lakowicz. Principles of fluorescence spectroscopy. Springer US, Boston, MA, 2006.

[2] John Frederick William Herschel. On a Case of Superficial Colour Presented by a Homogeneous Liquid Internally Colourless. Royal Society of London, 135(I):143-145, 1845.

[3] A. P. Silva, H. Q. Nimal Gunaratne, Thorfinnur Gunnlaugsson, Allen J. M. Huxley, Colin P. McCoy, Jude T. Rademacher, and Terence E. Rice. Signaling Recognition Events with Fluorescent Sensors and Switches. Chemical Reviews, 97(5):1515-1566, aug 1997.

[4] Jue Ling, Gaowa Naren, Jessica Kelly, Adam Qureshi, and A Prasanna De. Fluorescent logic systems for sensing and molecular computation : structure activity relationships in edge-detection. Faraday Discuss., 44(0):1-10, 2015.

[5] Zhiqian Guo, Sookil Park, Juyoung Yoon, and Injae Shin. Recent progress in the development of near-infrared fluorescent probes for bioimaging applications. Chemical Society reviews, 43(1):16-29, 2014. 
[6] Kensuke Komatsu, Yasuteru Urano, Hirotatsu Kojima, and Tetsuo Nagano. Development of an iminocoumarin-based zinc sensor suitable for ratiometric fluorescence imaging of neuronal zinc. Journal of the American Chemical Society, 129(44):13447-13454, nov 2007.

[7] Jessica P Miller, Christopher Egbulefu, Julie L Prior, Mingzhou Zhou, and Samuel Achilefu. Gradient-Based Algorithm for Determining Tumor Volumes in Small Animals Using Planar Fluorescence Imaging Platform. Tomography : a journal for imaging research, 2(1):17-25, mar 2016.

[8] Ellen K Feuster and Timothy E Glass. Detection of Amines and Unprotected Amino Acids in Aqueous Conditions by Formation of Highly Fluorescent Iminium Ions. Journal of the American Chemical Society, 125(52):16174-16175, 2003.

[9] Takahiro Egawa, Kenjiro Hanaoka, Yuichiro Koide, Sakiko Ujita, Naoya Takahashi, Yuji Ikegaya, Norio Matsuki, Takuya Terai, Tasuku Ueno, Toru Komatsu, and Tetsuo Nagano. Development of a far-red to near-infrared fluorescence probe for calcium ion and its application to multicolor neuronal imaging. Journal of the American Chemical Society, 133(36):14157-14159, sep 2011.

[10] Jun Yin, Ying Hu, and Juyoung Yoon. Fluorescent probes and bioimaging: alkali metals, alkaline earth metals and pH. Chemical Society Reviews, 44(14):4619-4644, 2015.

[11] Ernesto. Carafoli and Claude B. Klee. Calcium as a Cellular Regulator. Oxford University Press, 1999. 
[12] Lin Yuan, Weiying Lin, Kaibo Zheng, and Sasa Zhu. FRET-based smallmolecule fluorescent probes: Rational design and bioimaging applications. Accounts of Chemical Research, 46(7):1462-1473, 2013.

[13] J Steed, D Turner, and K Wallace. Core Concepts in Supramolecular Chemistry and Nanochemistry. John Wiley, 2008.

[14] Andrzej Sygula, Frank R. Fronczek, Renata Sygula, Peter W. Rabideau, and Marilyn M. Olmstead. A double concave hydrocarbon buckycatcher. Journal of the American Chemical Society, 129(13):3842-3843, apr 2007.

[15] Kenneth A. Connors. Binding Constants : The Measurement of Molecular Complex Stability, volume 47. Wiley, 1987.

[16] Justin A. Lemkul and David R. Bevan. Aggregation of alzheimer's amyloid $\beta$ peptide in biological membranes: A molecular dynamics study. Biochemistry, 52(29):4971-4980, jul 2013.

[17] G Brent Irvine, Omar M El-Agnaf, Ganesh M Shankar, and Dominic M Walsh. Protein Aggregation in the Brain: The Molecular Basis for Alzheimer's and Parkinson's Diseases. Molecular Medicine, 14(7-8):451-464, 2008.

[18] Amaresh Mishra, Rajani K. Behera, Pradipta K. Behera, Bijaya K. Mishra, and Gopa B. Behera. Cyanines during the 1990s: a review. Chemical Reviews, 100(6):1973-2011, 2000.

[19] Edwin E. Jelley. Spectral Absorption and Fluorescence of Dyes in the Molecular State. Nature, 138(3502):1009-1010, dec 1936. 
[20] A Eisfeld and J S Briggs. The J- and H-bands of organic dye aggregates. Chemical Physics, 324(2-3):376-384, 2006.

[21] Eion G. McRae and Michael Kasha. Enhancement of Phosphorescence Ability upon Aggregation of Dye Molecules. The Journal of Chemical Physics, 28(4):721, 1958.

[22] B Y Michael Kasha. Characterization of Electronic Transitions in Complex Molecules. Discuss. Faraday Soc., 9(c):14-19, 1950.

[23] Takashi Katoh, Yoshio Inagaki, and Renji Okazaki. Linear and Stack Oligostreptocyanines. Effects of Relative Orientation of Chromophores on Redox Potentials of Dye Aggregates. Bulletin of the Chemical Society of Japan, 70(9):2279-2286, sep 1997.

[24] Arnošt. Kotyk and J Slavik. Intracellular pH and Its Measurement. CRC Press, 1989.

[25] Frederick B Loiselle and Joseph R Casey. Measurement of Intracellular pH. Methods in molecular biology (Clifton, N.J.), 637:311-331, 2010.

[26] A Roos and W F Boron. Intracellular pH. Physiological reviews, 61(2):296-434, 1981.

[27] Elaine J Adie, S Kalinka, L Smith, M J Francis, A Marenghi, M E Cooper, M Briggs, N P Michael, G Milligan, and S Game. A pH-sensitive Fluor, CypHer5, Used to Monitor Agonist-Induced G Protein-Coupled Receptor Internalization in Live cells. BioTechniques, 33(5):1152-1157, 2002. 
[28] Marshall H. Montrose, Thomas Friedrich, and Heini Murer. Measurements of intracellular $\mathrm{pH}$ in single LLC-PK1 cells: Recovery from an acid load via basolateral $\mathrm{Na}+/ \mathrm{H}+$ exchange. The Journal of Membrane Biology, 97(1):6378, feb 1987.

[29] Hiroto Izumi, Takayuki Torigoe, Hiroshi Ishiguchi, Hidetaka Uramoto, Yoichiro Yoshida, Mizuho Tanabe, Tomoko Ise, Tadashi Murakami, Takeshi Yoshida, Minoru Nomoto, and Kimitoshi Kohno. Cellular pH regulators: Potentially promising molecular targets for cancer chemotherapy. Cancer Treatment Reviews, 29(6):541-549, 2003.

[30] T A Davies, R E Fine, R J Johnson, C A Levesque, W H Rathbun, K F Seetoo, S J Smith, G. Strohmeier, L. Volicer, L. Delva, and Et al. Non-age related differences in thrombin responses by platelets from male patients with advanced Alzheimer's disease. Biochem Biophys Res Commun, 194(1):537-543, 1993.

[31] Ralph Weissleder. A clearer vision for in vivo imaging. Nature biotechnology, 19(4):316-317, apr 2001.

[32] T J Rink, R Y Tsien, and T Pozzan. Cytoplasmic pH and Free Mg 2+ in Lymphocytes. J cell Biol, 95(c):189-196, 1982.

[33] Yasuteru Urano, Mako Kamiya, Kojiro Kanda, Tasuku Ueno, Kenzo Hirose, and Tetsuo Nagano. Evolution of fluorescein as a platform for finely tunable fluorescence probes. Journal of the American Chemical Society, 127(13):48884894, 2005. 
[34] Junyan Han and Kevin Burgess. Fluorescent indicators for intracellular pH. Chemical Reviews, 110(5):2709-2728, 2010.

[35] Yuichiro Koide, Yasuteru Urano, Kenjiro Hanaoka, Wen Piao, Moriaki Kusakabe, Nae Saito, Takuya Terai, Takayoshi Okabe, and Tetsuo Nagano. Development of NIR fluorescent dyes based on Si-rhodamine for in vivo imaging. Journal of the American Chemical Society, 134(11):5029-5031, 2012.

[36] Takuya Myochin, Kazuki Kiyose, Kenjiro Hanaoka, Hirotatsu Kojima, Takuya Terai, and Tetsuo Nagano. Rational design of ratiometric near-infrared fluorescent $\mathrm{pH}$ probes with various $\mathrm{pKa}$ values, based on aminocyanine. Journal of the American Chemical Society, 133(10):3401-3409, 2011.

[37] Kazuki Kiyose, Sakiko Aizawa, Eita Sasaki, Hirotatsu Kojima, Kenjiro Hanaoka, Takuya Terai, Yasuteru Urano, and Tetsuo Nagano. Molecular design strategies for near-infrared ratiometric fluorescent probes based on the unique spectral properties of aminocyanines. Chemistry - A European Journal, 15(36):9191-9200, 2009.

[38] Chunlong Sun, Peng Wang, Lushen Li, Gaoxin Zhou, Xi Zong, Bing Hu, Rui Zhang, Jin Cai, Junqing Chen, and Min Ji. A new near-infrared neutral pH fluorescent probe for monitoring minor $\mathrm{pH}$ changes and its application in imaging of HepG2 cells. Applied biochemistry and biotechnology, 172(2):1036-44, 2014.

[39] Wen Sun, Shigang Guo, Chong Hu, Jiangli Fan, and Xiaojun Peng. Recent Development of Chemosensors Based on Cyanine Platforms. Chemical Reviews, 116(14):7768-7817, jul 2016. 
[40] Michael E. Cooper, Susan Gregory, Elaine Adie, and Sian Kalinka. pH-Sensitive Cyanine Dyes for Biological Applications. Journal of Fluorescence, 12(3-4):425429, 2002.

[41] Zongren Zhang and Samuel Achilefu. Design, synthesis and evaluation of nearinfrared fluorescent $\mathrm{pH}$ indicators in a physiologically relevant range. Chemical communications (Cambridge, England), 0(47):5887-9, 2005.

[42] Bo Tang, Fabiao Yu, Ping Li, Lili Tong, Xia Duan, Ting Xie, and Xu Wang. A near-infrared neutral $\mathrm{pH}$ fluorescent probe for monitoring minor $\mathrm{pH}$ Changes: Imaging in living HepG2 and HL-7702Cells. Journal of the American Chemical Society, 131(8):3016-3023, mar 2009.

[43] Xiaole Shao. NIR Fluorescent Sensors for Blood pH and Fructose. PhD dissertation, University of Missouri, 2013.

[44] N. Sachedina and John C. Pickup. Performance assessment of the MedtronicMiniMed Continuous Glucose Monitoring System and its use for measurement of glycaemic control in Type 1 diabetic subjects. Diabetic Medicine, 20(12):10121015, dec 2003.

[45] John J. Mastrototaro. The MiniMed continuous glucose monitoring system. Diabetes Technol Ther, 2 Suppl 1(1):S13-8, dec 2000.

[46] K Satish, O Russell, and R Neil. Correlation of fingerstick blood glucose measurements with GlucoWatch ... Diabetes Care, 22(10), 1999. 
[47] J.a. Tamada, Satish Garg, Lois Jovanovic, K.R. Pitzer, Steve Fermi, R.O. Potts, and Others. Noninvasive glucose monitoring. JAMA: the journal of the American Medical Association, 282(19):1839, nov 1999.

[48] Huanfen Yao, Angela J. Shum, Melissa Cowan, Ilkka Lähdesmäki, and Babak A. Parviz. A contact lens with embedded sensor for monitoring tear glucose level. Biosensors and Bioelectronics, 26(7):3290-3296, 2011.

[49] Ramachandram Badugu, Joseph R. Lakowicz, and Chris D. Geddes. A Glucose Sensing Contact Lens: A Non-Invasive Technique for Continuous Physiological Glucose Monitoring. Journal of Fluorescence, 13(5):371-374, sep 2003.

[50] Timothy T Ruckh and Heather A Clark. Implantable nanosensors: Toward continuous physiologic monitoring. Analytical Chemistry, 86(3):1314-1323, feb 2014.

[51] Mary K. Balaconis, Yi Luo, and Heather A. Clark. Glucose-sensitive nanofiber scaffolds with an improved sensing design for physiological conditions. The Analyst, 140(3):716-23, 2015.

[52] Rohit Srivastava, Rahul Dev Jayant, Ayesha Chaudhary, and Michael J McShane. "Smart Tattoo" Glucose Biosensors and Effect of Coencapsulated AntiInflammatory Agents. Journal of Diabetes Science and Technology, 5(1):76-85, jan 2011.

[53] Jon Stefan Hansen, Jørn Bolstad Christensen, Johannes Fabritius Petersen, Thomas Hoeg-Jensen, and Jens Christian Norrild. Arylboronic acids: A diabetic eye on glucose sensing. Sensors and Actuators, B: Chemical, 161(1):45-79, 2012. 
[54] John C. Pickup, Faeiza Hussain, Nicholas D. Evans, Olaf J. Rolinski, and David J S Birch. Fluorescence-based glucose sensors. Biosensors and Bioelectronics, 20(12):2555-2565, 2005.

[55] M. I. Wolfrom and J. Solms. Phenylboronates of Pentoses and 6-Deoxyhexoses. The Journal of Organic Chemistry, 21(7):815-816, jul 1956.

[56] Greg Springsteen and Binghe Wang. A detailed examination of boronic acid-diol complexation. Tetrahedron, 58(26):5291-5300, 2002.

[57] Nicolas DiCesare and Joseph R. Lakowicz. Spectral properties of fluorophores combining the boronic acid group with electron donor or withdrawing groups. Implication in the development of fluorescence probes for saccharides. Journal of Physical Chemistry A, 105(28):6834-6840, 2001.

[58] Nicolas Di Cesare and Joseph R Lakowicz. Wavelength-ratiometric probes for saccharides based on donoracceptor diphenylpolyenes. Journal of Photochemistry and Photobiology A: Chemistry, 143(1):39-47, 2001.

[59] Tony D. James, K. R. A. Samankumara Sandanayake, and Seiji Shinkai. Novel photoinduced electron-transfer sensor for saccharides based on the interaction of boronic acid and amine. Chemical Communications, 0(4):477-478, 1994.

[60] Tony D. James, K. R. A. Samankumara Sandanayake, Ritsuko Iguchi, and Seiji Shinkai. Novel Saccharide-Photoinduced Electron-Transfer Sensors Based on the Interaction of Boronic Acid and Amine. Journal of the American Chemical Society, 117(35):8982-8987, sep 1995. 
[61] Susumu Arimori, Michael L. Bell, Chan S. Oh, and Tony D. James. A modular fluorescence intramolecular energy transfer saccharide sensor. Organic Letters, $4(24): 4249-4251,2002$.

[62] Susumu Arimori, Michael L. Bell, Chan S. Oh, Karine A. Frimat, and Tony D. James. Modular fluorescence sensors for saccharides. Journal of the Chemical Society, Perkin Transactions 1, 218(6):803-808, mar 2002.

[63] Wei Yang, Huan He, and Dale G. Drueckhammer. Computer-guided design in molecular recognition: Design and synthesis of a glucopyranose receptor. Angewandte Chemie - International Edition, 40(9):1714-1718, may 2001.

[64] Mia Bielecki, Hanne Eggert, and Jens Chr. Norrild. A fluorescent glucose sensor binding covalently to all five hydroxy groups of $\alpha$-D-glucofuranose. A reinvestigation. Journal of the Chemical Society, Perkin Transactions 2, 0(3):449-456, 1999.

[65] Kyungsuk Yum, Thomas P McNicholas, Bin Mu, and Michael S Strano. Singlewalled carbon nanotube-based near-infrared optical glucose sensors toward in vivo continuous glucose monitoring. Journal of diabetes science and technology, 7(1):72-87, jan 2013.

[66] Yumei Hu, Xiaomei Jiang, Laiying Zhang, Jiao Fan, and Weitai Wu. Construction of near-infrared photonic crystal glucose-sensing materials for ratiometric sensing of glucose in tears. Biosensors and Bioelectronics, 48:94-99, 2013. 
[67] Ziping Liu, Linlin Liu, Minghui Sun, and Xingguang Su. A novel and convenient near-infrared fluorescence "turn off-on" nanosensor for detection of glucose and fluoride anions. Biosensors and Bioelectronics, 65:145-151, 2015.

[68] Faaizah Khan and John C. Pickup. Near-infrared fluorescence glucose sensing based on glucose/galactose-binding protein coupled to 651-Blue Oxazine. Biochemical and Biophysical Research Communications, 438(3):488-492, 2013.

[69] Roger R Nani, James B Shaum, Alexander P Gorka, and Martin J Schnermann. Electrophile-integrating Smiles rearrangement provides previously inaccessible C4'-O-alkyl heptamethine cyanine fluorophores. Organic letters, 17(2):302-5, 2015 .

[70] Jun Yan, Greg Springsteen, Susan Deeter, and Binghe Wang. The relationship among $\mathrm{pKa}, \mathrm{pH}$, and binding constants in the interactions between boronic acids and diolsit is not as simple as it appears. Tetrahedron, 60(49):1120511209, 2004.

[71] Vikram J. Pansare, Shahram Hejazi, William J. Faenza, and Robert K. Prud'Homme. Review of long-wavelength optical and NIR imaging materials: Contrast agents, fluorophores, and multifunctional nano carriers. Chemistry of Materials, 24(5):812-827, mar 2012.

[72] Toshiyuki Kowada, Hiroki Maeda, and Kazuya Kikuchi. BODIPY-based probes for the fluorescence imaging of biomolecules in living cells. Chemical Society reviews, 44(14):4953-72, 2015. 
[73] Sarah C Ritter, Mark A Milanick, and Kenith E Meissner. Encapsulation of FITC to monitor extracellular pH: a step towards the development of red blood cells as circulating blood analyte biosensors. Biomedical optics express, 2(7):2012-21, jul 2011.

[74] Sarah C. Ritter, Xiaole Shao, Nicholas Cooley, Mark A. Milanick, Timothy E. Glass, and Kenith E. Meissner. Blood analyte sensing using fluorescent dyeloaded red blood cells. SPIE Proceedings, 8951:895109, feb 2014.

[75] Mark A. Milanick, Sarah Ritter, and Kenith Meissner. Engineering erythrocytes to be erythrosensors: First steps. Blood Cells, Molecules, and Diseases, 47(2):100-106, 2011.

[76] Carlos H. Villa, Aaron C. Anselmo, Samir Mitragotri, and Vladimir Muzykantov. Red blood cells: Supercarriers for drugs, biologicals, and nanoparticles and inspiration for advanced delivery systems. Advanced Drug Delivery Reviews, 106:88-103, 2016.

[77] G. Schwoch and H. Passow. Preparation and properties of human erythrocyte ghosts. Molecular and Cellular Biochemistry, 2(2):197-218, dec 1973.

[78] Carmen Gutiérrez Millán, Aránzazu Zarzuelo Castañeda, Ma. Luisa Sayalero Marinero, and José M. Lanao. Factors associated with the performance of carrier erythrocytes obtained by hypotonic dialysis. Blood Cells, Molecules, and Diseases, 33(2):132-140, 2004.

[79] M. E. Favretto, J. C A Cluitmans, G. J C G M Bosman, and R. Brock. Human erythrocytes as drug carriers: Loading efficiency and side effects of hypotonic 
dialysis, chlorpromazine treatment and fusion with liposomes. Journal of Controlled Release, 170(3):343-351, 2013.

[80] Huining He, Junxiao Ye, Yinsong Wang, Quan Liu, Hee Sun Chung, Young Min Kwon, Meong Cheol Shin, Kyuri Lee, and Victor C. Yang. Cell-penetrating peptides meditated encapsulation of protein therapeutics into intact red blood cells and its application. Journal of Controlled Release, 176(1):123-132, 2014.

[81] Jiahai Shi, Lenka Kundrat, Novalia Pishesha, Angelina Bilate, Chris Theile, Takeshi Maruyama, Stephanie K Dougan, Hidde L Ploegh, and Harvey F Lodish. Engineered red blood cells as carriers for systemic delivery of a wide array of functional probes. Proceedings of the National Academy of Sciences of the United States of America, 111(28):10131-6, jul 2014.

[82] Sergei Zaitsev, M. Anna Kowalska, Michael Neyman, Ronald Carnemolla, Samira Tliba, Bi Sen Ding, Aaron Stonestrom, Dirk Spitzer, John P. Atkinson, Mortimer Poncz, Douglas B. Cines, Charles T. Esmon, and Vladimir R. Muzykantov. Targeting recombinant thrombomodulin fusion protein to red blood cells provides multifaceted thromboprophylaxis. Blood, 119(20):4779$4785,2012$.

[83] Marie-Catherine Giarratana, Ladan Kobari, Hélène Lapillonne, David Chalmers, Laurent Kiger, Thérèse Cynober, Michael C Marden, Henri Wajcman, and Luc Douay. Ex vivo generation of fully mature human red blood cells from hematopoietic stem cells. Nature biotechnology, 23(1):69-74, jan 2005.

[84] Luciana Chessa, Vincenzo Leuzzi, Alessandro Plebani, Annarosa Soresina, Roberto Micheli, Daniela D'Agnano, Tullia Venturi, Anna Molinaro, Elisa Fazzi, 
Mirella Marini, Pierino Ferremi Leali, Isabella Quinti, Filomena Monica Cavaliere, Gabriella Girelli, Maria Cristina Pietrogrande, Andrea Finocchi, Stefano Tabolli, Damiano Abeni, and Mauro Magnani. Intra-erythrocyte infusion of dexamethasone reduces neurological symptoms in ataxia teleangiectasia patients: results of a phase 2 trial. Orphanet journal of rare diseases, 9(1):5, 2014.

[85] Vincenzo Leuzzi, Roberto Micheli, Daniela D’Agnano, Anna Molinaro, Tullia Venturi, Alessandro Plebani, Annarosa Soresina, Mirella Marini, Pierino Ferremi Leali, Isabella Quinti, Maria C. Pietrogrande, Andrea Finocchi, Elisa Fazzi, Luciana Chessa, and Mauro Magnani. Positive effect of erythrocytedelivered dexamethasone in ataxia-telangiectasia. Neurology neuroimmunology Es neuroinflammation, 2(3):e98, jun 2015.

[86] Mathilde Hunault-Berger, Thibaut Leguay, Françoise Huguet, Stéphane Leprêtre, Eric Deconinck, Mario Ojeda-Uribe, Caroline Bonmati, Martine Escoffre-Barbe, Pierre Bories, Chantal Himberlin, Patrice Chevallier, Philippe Rousselot, Oumedaly Reman, Marie Laure Boulland, Severine Lissandre, Pascal Turlure, Didier Bouscary, Laurence Sanhes, Ollivier Legrand, Marina LafagePochitaloff, Marie C Béné, David Liens, Yann Godfrin, Norbert Ifrah, and Hervé Dombret. A Phase 2 study of L-asparaginase encapsulated in erythrocytes in elderly patients with Philadelphia chromosome negative acute lymphoblastic leukemia: The GRASPALL/GRAALL-SA2-2008 study. American Journal of Hematology, 90(9):811-818, sep 2015.

[87] J B Bachet, Fabien Gay, R Marechal, M P Galais, Antoine Adenis, C Ds 
Ms, Jerome Cros, Pieter Demetter, Magali Svrcek, Armelle Bardier-Dupas, J F Emile, Pascal Hammel, Christelle Ebenezer, Willy Berlier, Yann Godfrin, and T Andre. Asparagine Synthetase Expression and Phase I Study With LAsparaginase Encapsulated in Red Blood Cells in Patients With Pancreatic Adenocarcinoma. Pancreas, 44(7):1141-1147, oct 2015.

[88] Maximilian Wei-Lin Popp and Hidde L. Ploegh. Making and Breaking Peptide Bonds: Protein Engineering Using Sortase. Angewandte Chemie International Edition, 50(22):5024-5032, may 2011.

[89] Sergei Zaitsev, Dirk Spitzer, Juan Carlos Murciano, Bi Sen Ding, Samira Tliba, M. Anna Kowalska, Oscar A. Marcos-Contreras, Alice Kuo, Victoria Stepanova, John P. Atkinson, Mortimer Poncz, Douglas B. Cines, and Vladimir R. Muzykantov. Sustained thromboprophylaxis mediated by an RBCtargeted pro-urokinase zymogen activated at the site of clot formation. Blood, 115(25):5241-5248, 2010.

[90] Juan-Carlos Murciano, Sandra Medinilla, Donald Eslin, Elena Atochina, Douglas B Cines, and Vladimir R Muzykantov. Prophylactic fibrinolysis through selective dissolution of nascent clots by tPA-carrying erythrocytes. Nature biotechnology, 21(8):891-896, aug 2003.

[91] Marina Inmaculada Garín, Rosa María López, Silvia Sanz, Montserrat Pinilla, and José Luque. Erythrocytes as carriers for recombinant human erythropoietin. Pharmaceutical Research, 13(6):869-874, 1996. 
[92] Garret M Ihler, Robert H Glew, and Frederick W Schnure. Enzyme Loading of Erythrocytes. Proceedings of the National Academy of Sciences, 70(9):26632666, 1973.

[93] S J Updike, R T Wakamiya, and E N Lightfoot Jr. Asparaginase entrapped in red blood cells: action and survival. Science, 193(4254):681-683, 1976.

[94] Mehrdad Hamidi, Najmeh Zarei, Abdolhossein Zarrin, and Soleiman Mohammadi-Samani. Preparation and validation of carrier human erythrocytes loaded by bovine serum albumin as a model antigen/protein. Drug delivery, 14(5):295-300, jan 2007.

[95] Ryo Taguchi, Koichi Suzuki, Toshikatsu Nakabayashi, and Hiroh Ikezawa. Acetylcholinesterase Release from Mammalian Erythrocytes by Phosphatidylinositol-Specific Phospholipase $\mathrm{C}$ of Bacillus thuringiensis and Characterization of the Released Enzyme. The Journal of Biochemistry, 96(2):437-446, 1984 .

[96] F Worek, G Reiter, P. Eyer, and L. Szinicz. Reactivation kinetics of acetylcholinesterase from different species inhibited by highly toxic organophosphates. Archives of Toxicology, 76(9):523-529, 2002.

[97] Philip L Barton, Anthony H Futerman, and I Silman. Arrhenius plots of acetylcholinesterase activity in mammalian erythrocytes and in Torpedo electric organ. Effect of solubilization by proteinases and by a phosphatidylinositol-specific phospholipase C. The Biochemical journal, 231(1):237-240, 1985. 
[98] Robert C. Carlisle, Ying Di, Anna M. Cerny, Andreas F.-P. Sonnen, Robert B. Sim, Nicola K. Green, Vladimir Subr, Karel Ulbrich, Robert J. C. Gilbert, Kerry D. Fisher, Robert W. Finberg, and Leonard W. Seymour. Human erythrocytes bind and inactivate type 5 adenovirus by presenting Coxsackie virusadenovirus receptor and complement receptor 1. Blood, 113:1909-1918, 2009.

[99] C. S. Giampapa, Soman N Abraham, Thomas M Chiang, and E. H. Beachey. Isolation and characterization of a receptor for type 1 fimbriae of Escherichia coli from guinea pig erythrocytes. Journal of Biological Chemistry, 263(11):5362$5367,1988$.

[100] Selasi Dankwa, Caeul Lim, Amy K. Bei, Rays H. Y. Jiang, James R. Abshire, Saurabh D. Patel, Jonathan M. Goldberg, Yovany Moreno, Maya Kono, Jacquin C. Niles, and Manoj T. Duraisingh. Ancient human sialic acid variant restricts an emerging zoonotic malaria parasite. Nature Communications, 7:11187, apr 2016.

[101] Robert Flower, Enrico Peiretti, Mauro Magnani, Luigia Rossi, Sonja Serafini, Zygmunt Gryczynski, and Ignacy Gryczynski. Observation of erythrocyte dynamics in the retinal capillaries and choriocapillaris using ICG-loaded erythrocyte ghost cells. Investigative Ophthalmology and Visual Science, 49(12):55105516 , dec 2008.

[102] Vanessa Bourgeaux, José M. Lanao, Bridget E. Bax, and Yann Godfrin. Drugloaded erythrocytes: On the road toward marketing approval. Drug Design, Development and Therapy, 10:665-676, feb 2016. 
[103] Georgeta Crivat and Justin W Taraska. Imaging proteins inside cells with fluorescent tags. Trends in Biotechnology, 30(1):8-16, jan 2012.

[104] Lin Xue, Efthymia Prifti, and Kai Johnsson. A General Strategy for the Semisynthesis of Ratiometric Fluorescent Sensor Proteins with Increased Dynamic Range. Journal of the American Chemical Society, 138(16):5258-5261, apr 2016.

[105] Guillaume F. Rousseau, Marie-Catherine Giarratana, and Luc Douay. Largescale production of red blood cells from stem cells: What are the technical challenges ahead? Biotechnology Journal, 9(1):28-38, jan 2014. 


\section{VITA}

Nicholas was born in Harristown, Pennsylvania but grew up in the middle of farm country north of Dayton, Ohio. After high school he attended The Ohio State University, receiving undergraduate degrees in Molecular Genetics and Microbiology. He entered grad school in the fall of 2009 and joined Dr. Timothy E. Glass's research group the following spring. His time in the Glass Lab has been spent synthesizing and testing fluorescent chemical sensors.

Upon completion of his $\mathrm{PhD}$, Nicholas has accepted a postdoctoral position with Dr. Erik Wright at the University of Pittsburgh. He will be continuing on his interdisciplinary track to work on 'big data' approaches to exploring antibiotic resistance and secondary metabolite identification. 